\title{
Type Iln supernova light-curve properties measured from an untargeted survey sample ${ }^{\star, \star \star, \star \star \star \star}$
}

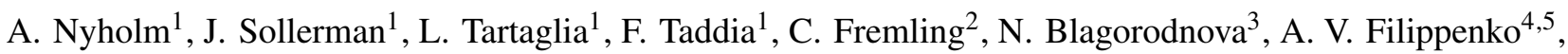 \\ A. Gal-Yam ${ }^{6}$, D. A. Howell ${ }^{7,8}$, E. Karamehmetoglu ${ }^{1}$, S. R. Kulkarni ${ }^{2}$, R. Laher ${ }^{9}$, G. Leloudas ${ }^{10}$, F. Masci ${ }^{9}$, \\ M. M. Kasliwal ${ }^{2}$, K. Morå ${ }^{11}$, T. J. Moriya ${ }^{12}$, E. O. Ofek ${ }^{13}$, S. Papadogiannakis ${ }^{11}$, R. Quimby ${ }^{14,15}$, \\ U. Rebbapragada ${ }^{16}$, and S. Schulze ${ }^{6}$ \\ (Affiliations can be found after the references)
}

Received 13 June 2019 / Accepted 30 September 2019

\begin{abstract}
The evolution of a Type IIn supernova (SN IIn) is governed by the interaction between the SN ejecta and a hydrogen-rich circumstellar medium. The SNe IIn thus allow us to probe the late-time mass-loss history of their progenitor stars. We present a sample of SNe IIn from the untargeted, magnitude-limited surveys of the Palomar Transient Factory (PTF) and its successor, the intermediate PTF (iPTF). To date, statistics on SN IIn optical light-curve properties have generally been based on small $(\$ 10 \mathrm{SNe})$ samples from targeted SN surveys. The SNe IIn found and followed by the PTF/iPTF were used to select a sample of 42 events with useful constraints on the rise times as well as with available post-peak photometry. The sample SNe were discovered in 2009-2016 and have at least one low-resolution classification spectrum, as well as photometry from the P48 and P60 telescopes at Palomar Observatory. We study the light-curve properties of these SNe IIn using spline fits (for the peak and the declining portion) and template matching (for the rising portion). We study the peak-magnitude distribution, rise times, decline rates, colour evolution, host galaxies, and K-corrections of the SNe in our sample. We find that the typical rise times are divided into fast and slow risers at $20 \pm 6 \mathrm{~d}$ and $50 \pm 11 \mathrm{~d}$, respectively. The decline rates are possibly divided into two clusters (with slopes $0.013 \pm 0.006 \mathrm{mag} \mathrm{d}^{-1}$ and $0.040 \pm$ $0.010 \mathrm{mag} \mathrm{d}^{-1}$ ), but this division has weak statistical significance. We find no significant correlation between the peak luminosity of SNe IIn and their rise times, but the more luminous SNe IIn are generally found to be more long-lasting. Slowly rising SNe IIn are generally found to decline slowly. The SNe in our sample were hosted by galaxies of absolute magnitude $-22 \lesssim M_{\mathrm{g}} \lesssim-13$ mag. The K-corrections at light-curve peak of the SNe IIn in our sample are found to be within 0.2 mag for the observer's frame $r$-band, for SNe at redshifts $z<0.25$. By applying K-corrections and also including ostensibly "superluminous" SNe IIn, we find that the peak magnitudes are $M_{\text {peak }}^{r}=-19.18 \pm 1.32$ mag. We conclude that the occurrence of conspicuous light-curve bumps in SNe IIn, such as in iPTF13z, are limited to $1.4_{-1.0}^{+14.6} \%$ of the SNe IIn. We also investigate a possible sub-type of SNe IIn with a fast rise to a $\gtrsim 50 \mathrm{~d}$ plateau followed by a slow, linear decline.
\end{abstract}

Key words. supernovae: general

\section{Introduction}

A supernova (SN) that has a spectrum showing Balmer emission lines with narrow or intermediate-width central components and broad line wings is classified as a Type IIn SN. This SN classification (where "II" indicates presence of hydrogen and " $n$ " stands for narrow) was proposed by Schlegel (1990) and reached wider use via the review by Filippenko (1997). It has been shown in observations and modelling (e.g. Chevalier \& Fransson 1994; Smith 2017a; Dessart et al. 2016) that the SN IIn spectral signature arises from the interaction of $\mathrm{SN}$ ejecta with a hydrogenrich circumstellar medium (CSM), where shocks form when the ejecta sweep up the CSM, converting the kinetic energy of the ejecta into radiated energy.

An SN IIn spectrum is produced in a process involving the SN environment, and shows little direct signatures of the explosion itself. Many different scenarios can therefore potentially give rise to $\mathrm{SN}$ IIn spectral signatures, making it diffi-

\footnotetext{
* Full Table 2 is only available at the CDS via anonymous ftp to cdsarc.u-strasbg. fr (130.79.128.5) or via http://cdsarc. u-strasbg.fr/viz-bin/cat/J/A+A/637/A73

$\star \star$ The classification spectra and the photometry are available via the Weizmann Interactive Supernova Data Repository (WISeREP) at https: //wiserep. weizmann.ac . il

$\star \star \star$ Based on observations made with the Palomar Transient Factory and intermediate Palomar Transient Factory surveys.
}

cult to tell whether a core-collapse (CC) SN explosion or a violent, but nondisruptive, stellar outburst sends the ejecta off (e.g. Dessart et al. 2009). Whereas the ejecta-CSM interaction makes SNe IIn useful probes of progenitor mass-loss histories, $\mathrm{SNe}$ IIn are also challenging to understand since the nature of the underlying energy source remains elusive. The spectroscopic criteria defining the SN IIn classification makes them spectroscopically somewhat similar, but their peak absolute magnitudes vary greatly, as do their light-curve shapes and the presence of undulations and bumps in their light-curves. The wide range of light-curve properties shown by $\mathrm{SNe}$ IIn indicates significant variety in both CSM and ejecta properties (e.g. Moriya \& Maeda 2014), suggesting that different mechanisms and progenitor channels lead to the SNe IIn we observe (Smith 2014).

$\mathrm{SNe}$ IIn are intrinsically rare. A volume-limited SN sample from the targeted Lick Observatory Supernova Search (LOSS; Li et al. 2011) shows that $\sim 7 \%$ of all CC SNe are SNe IIn. Their diverse properties call for large samples in order to improve our understanding of this SN type. However, the current SN IIn literature is skewed towards a small number of well-studied events, which are often nearby (at redshifts $z \lesssim 0.02$ ) or characterised by some unusual property. Our aim in this paper is to study a sample of SNe IIn from the untargeted Palomar Transient Factory (PTF; Law et al. 2009) survey and its successor, the intermediate PTF (iPTF; Kulkarni 2013), with emphasis on the SN IIn optical light-curves. 
Table 1. Sample studies of SNe IIn based on optical observations.

\begin{tabular}{|c|c|c|c|c|c|c|}
\hline Reference & $\begin{array}{l}\text { Sample } \\
\text { size }\end{array}$ & $\begin{array}{l}\text { Lowest } z \\
\text { in sample }\end{array}$ & $\begin{array}{l}\text { Median } z \\
\text { in sample }\end{array}$ & $\begin{array}{l}\text { Maximum } z \\
\text { in sample }\end{array}$ & $\begin{array}{l}\text { Mode of sample } \\
\text { source survey(s) }\end{array}$ & $\begin{array}{l}\text { Main area } \\
\text { of study }\end{array}$ \\
\hline Li et al. (2002) & 7 & 0.0022 & 0.0047 & 0.018 & Targeted & Late-time photometry \\
\hline Richardson et al. (2002) & 9 & 0.0028 & 0.0082 & 0.043 & Targeted & Luminosity function \\
\hline Li et al. (2011) & 7 & 0.0024 & 0.0088 & 0.012 & Targeted & Luminosity function \\
\hline Kiewe et al. (2012) & 4 & 0.015 & 0.024 & 0.031 & Targeted & Light-curves and spectra \\
\hline Taddia et al. (2013) & 7 & 0.0071 & 0.017 & 0.029 & Mainly targeted & Light-curves and spectra \\
\hline Ofek et al. (2014c) & 19 & 0.031 & 0.073 & 0.28 & Untargeted & Rise times \\
\hline Ofek et al. (2014a) & 16 & 0.004 & 0.031 & 0.14 & Mainly untargeted & Precursor outbursts \\
\hline Richardson et al. (2014) & 31 & 0.0021 & 0.016 & 0.35 & Mainly targeted & Luminosity function \\
\hline Bilinski et al. (2015) & 6 & 0.0061 & 0.0095 & 0.011 & Targeted & Precursor outbursts \\
\hline de la Rosa et al. (2016) & 8 & 0.004 & 0.01 & 0.017 & Mainly targeted & Light-curves \\
\hline This work & 42 & 0.014 & 0.078 & 0.313 & Untargeted & Light-curves \\
\hline
\end{tabular}

Notes. The works included in the table all discuss photometric properties of SNe IIn. For the sample size counts, the SNe labelled as SNe IIn by the respective authors were used. If available, the redshifts $(z)$ of the SNe in each sample were taken from the respective papers. Otherwise, distance moduli or luminosity distances given in the papers were converted to redshifts using cosmological parameters from Sect. 3 without infall correction. Li et al. (2002) gives no distance information for their sample SNe; here, NED redshifts for their respective SN host galaxies were used.

Among the SN IIn samples presented in the literature are works concerning SN data releases and analyses (e.g. Kiewe et al. 2012; Taddia et al. 2013, 2015a), and also environmental (e.g. Kelly \& Kirshner 2012; Habergham et al. 2014; Taddia et al. 2015a; Galbany et al. 2018; Kuncarayakti et al. 2018) and SN rate studies (Li et al. 2011), as well as precursor outburst analyses (e.g. Ofek et al. 2014a; Bilinski et al. 2015). In Table 1 we present a summary of the SN IIn samples in the literature concerning optical light-curve properties.

The decline behaviour after peak brightness differs considerably among SNe IIn. Among slowly declining SNe IIn, SN $1988 Z$ has become a benchmark event in the literature (Stritzinger et al. 2012; Habergham et al. 2014; Turatto et al. 1993). Other SNe IIn decline considerably faster, with SN 1998S (Liu et al. 2000; Fassia et al. 2000) commonly given as a specimen. Apart from decline rates, light-curve shapes can also distinguish SNe IIn. SN 1994W (Sollerman et al. 1998) often exemplifies SNe IIn with plateaus in their light-curves followed by a sharp decline in brightness, sometimes called SNe IIn-P (Mauerhan et al. 2013a). Some SNe IIn exhibit distinct episodes of re-brightening ("bumps"), thus breaking their decline in brightness (e.g. Stritzinger et al. 2012; Graham et al. 2014; Martin et al. 2015; Nyholm et al. 2017).

An important finding facilitated by modern surveys, such as the PTF, is that some SNe IIn have precursor outbursts in the years before the main SN outburst. Sample studies (Ofek et al. 2014a; Bilinski et al. 2015) give inconclusive results regarding whether precursor events are common (possibly owing to the different methods used in the respective studies). Precursor outbursts suggest connections between SNe IIn and phenomena such as luminous blue variable (LBV; Humphreys \& Davidson 1994) outbursts (Smith et al. 2011) as well as other so called SN impostors (Van Dyk et al. 2000; Van Dyk \& Matheson 2012).

The SN IIn samples in the literature have uneven photometric coverage of the $\mathrm{SNe}$ during the rising phase of their light-curves. A number of the benchmark SN IIn events (e.g. SN 1988Z, Stathakis \& Sadler 1991; SN 1995N, Fransson et al. 2002; SN 2005ip and SN 2006jd, Stritzinger et al. 2012) lack well-determined rise times to peak. The literature sample of light-curves shown by Taddia et al. (2015a, their Fig. 10) also indicates the lack of pre-peak photometry for a number of oth- erwise well-characterised SNe IIn. The existence of a correlation between rise time and peak luminosity for SNe IIn has been investigated (Ofek et al. 2014b,c; Moriya \& Maeda 2014) and suggested to exist (Ofek et al. 2014c). To further study this possible correlation, more knowledge of SNe IIn rise times is needed.

By using a sample from the high-cadence and untargeted PTF/iPTF search to study light-curve rise times, peak absolute magnitudes, and light-curve slopes during the decline phase, we can characterise the SN IIn type. The untargeted nature of the PTF/iPTF survey provides a sample which is not biased towards $\mathrm{SNe}$ in luminous, metal-rich, high-star-formation-rate host galaxies. The high cadence of PTF/iPTF also allows us to put tighter constraints on the rise times of our SNe.

In Sect. 2, we present the SN search and follow-up observations done by the PTF/iPTF and the telescopes and instruments used, as well as the reduction methods adopted to prepare the photometric data for analysis. We discuss the selection of our SN IIn sample as well as its properties in Sect. 3, including a discussion of distances and foreground extinction of the SNe. In Sect. 4, we measure the light-curve peak magnitudes and peak epochs (Sect. 4.1) and the decline rates of the SNe (Sect. 4.2). We also study the light-curve rise times (Sect. 4.3), as well as possible correlations between these light-curve-shape parameters (Sect. 4.4). Colour curves are examined using our optical photometry (Sect. 4.5). The luminosity function is examined (Sect. 4.6). A simple study of the host galaxies of our sample $\mathrm{SNe}$ is done, using Sloan Digital Sky Survey (SDSS) and Panoramic Survey Telescope and Rapid Response System (Pan-STARRS) photometry for the hosts (Sect. 4.7). In Sect. 5, we discuss our results and highlight some sample SNe with interesting properties, and estimate the fraction of SNe IIn having light-curve bumps. We summarise our conclusions in Sect. 6. In Appendix A, we show compilations of spectra and light-curves. In Appendix B, we use the spectra of our SNe to study their K-corrections.

\section{Observations and data reduction}

The PTF and iPTF surveys did untargeted searches for astronomical transients during 2009-2017, using the $1.2 \mathrm{~m}$ Samuel Oschin telescope (known as P48) at Palomar Observatory. P48 equipped with the CFH12K mosaic CCD gave a $7.26 \mathrm{deg}^{2}$ field 
Table 2. Photometry of the $42 \mathrm{SNe}$ IIn in the sample.

\begin{tabular}{|c|c|c|c|c|c|c|c|c|c|}
\hline $\mathrm{SN}$ & $\begin{array}{l}\text { Epoch } \\
\text { (JD) }\end{array}$ & $\begin{array}{l}\text { Magnitude } \\
\text { (AB) }\end{array}$ & $\begin{array}{l}\text { Magnitude } \\
\text { error }\end{array}$ & $\begin{array}{l}\text { Limiting } \\
\text { magnitude }\end{array}$ & Telescope & Camera & Filter & $\begin{array}{c}\text { Reference } \\
\text { image }\end{array}$ & Pipeline \\
\hline PTF09tm & 2454959.6605 & 99.00 & 99.0 & 20.02 & P48 & CFH12K & $R$ & P48ref & PTFIDE \\
\hline PTF09tm & 2454959.7523 & 99.00 & 99.0 & 20.26 & P48 & CFH12K & $R$ & P48ref & PTFIDE \\
\hline$\ldots$ & $\ldots$ & $\ldots$ & $\ldots$ & $\ldots$ & $\ldots$ & & $\cdots$ & $\ldots$ & \\
\hline PTF09tm & 2455005.8071 & 18.87 & 0.09 & 99.00 & P48 & CFH12K & $R$ & P48ref & PTFIDE \\
\hline PTF09tm & 2455005.8816 & 18.83 & 0.09 & 99.00 & P48 & CFH12K & $R$ & P48ref & PTFIDE \\
\hline$\ldots$ & $\ldots$ & $\ldots$ & $\ldots$ & $\ldots$ & $\ldots$ & & & $\cdots$ & \\
\hline PTF10weh & 2455724.9677 & 21.46 & 0.13 & 99.00 & P60 & GRBCam & $r$ & SDSSref & FPipe \\
\hline PTF10weh & 2455481.6718 & 18.69 & 0.01 & 99.00 & P60 & GRBCam & $i$ & SDSSref & FPipe \\
\hline$\ldots$ & $\ldots$ & $\ldots$ & $\ldots$ & $\ldots$ & $\ldots$ & $\ldots$ & $\ldots$ & $\ldots$ & $\ldots$ \\
\hline PTF11qnf & 2456037.7320 & 19.99 & 0.09 & 99.00 & P60 & GRBCam & $r$ & PS1ref & FPipe \\
\hline$\ldots$ & $\ldots$ & $\ldots$ & $\ldots$ & $\ldots$ & $\ldots$ & $\ldots$ & $\ldots$ & $\ldots$ & $\ldots$ \\
\hline iPTF15aym & 2457480.9090 & 21.31 & 0.06 & 99.00 & P60 & SEDM & $g$ & SDSSref & FPipe \\
\hline$\ldots$ & $\ldots$ & $\ldots$ & $\ldots$ & $\ldots$ & $\ldots$ & $\ldots$ & $\ldots$ & $\ldots$ & $\ldots$ \\
\hline
\end{tabular}

Notes. All magnitudes reported are AB magnitudes from PSF photometry on reference subtracted images. The columns give the name of the SN, the Julian Date (JD) of the photometry, the magnitude and its $1 \sigma$ error (if SN detected, otherwise given as "99") or the upper limit (magnitudes, if SN not detected, otherwise given as "99"), the telescope used (P48 or P60), the camera used (CFH12K, GRBCam or SEDM), the filter used (Mould $R$ or SDSS $g$ for P48, SDSS griz or Johnson $B$ for P60), the source of the reference image (P48, P60, SDSS or PS1 i.e. Pan-STARRS) and the pipeline used for the photometry (PTFIDE or FPipe). FPipe uses SDSS stars as reference stars, or PS1 stars when outside the SDSS footprint. Details on instruments etc. are given in Sect. 2. Photometric upper limits from PTFIDE are given at $5 \sigma$ level. Upper limits from FPipe are given at $3 \sigma$ for GRBCam and at $5 \sigma$ for SEDM, respectively. Selected parts of the table are shown here, as a guide to its content. The full table is available at the CDS.

of view (Law et al. 2009). Images were taken through either a Mould $R$-band filter (Law et al. 2009; Ofek et al. 2012; Laher et al. 2014) or an SDSS $g$-band filter (Fukugita et al. 1996), giving a limiting magnitude of $R \approx 20.5$ or $g \approx 21$ (respectively) under typical conditions. After image processing and an automatic initial selection of candidates (Cao et al. 2016), a human scanner vetted the images and flagged interesting candidates for follow-up photometry and spectroscopy on other telescopes. All SNe in the sample presented in this paper were found independently with the P48. Whereas several different pipelines were used for photometry in P48 images during the course of PTF/iPTF, all SN photometry from P48 presented in this paper has been extracted with the PTFIDE (PTF Image Differencing and Extraction; Masci et al. 2017) pipeline.

An important instrument for photometric follow-up observations during PTF/iPTF was the $1.52 \mathrm{~m}$ telescope (known as P60) at Palomar Observatory. The telescope was used for imaging with the GRBCam camera (Cenko et al. 2006) or the Spectral Energy Distribution Machine (SEDM) integral field spectrograph (Blagorodnova et al. 2018) using SDSS gri filters. A typical strategy with the P60 was to obtain SDSS gri images of a SN field with a cadence of $1 \mathrm{~d}$ or $3 \mathrm{~d}$ (for young $\mathrm{SNe}$ ) and a cadence of $6 \mathrm{~d}$ for older SNe. Other cadences and filters (for example SDSS $z$ and Johnson $B$ ) were also sometimes used. For this paper, SN photometry of the images from P60 (both GRBCam and SEDM) was done with the FPipe pipeline (Fremling et al. 2016). All magnitudes reported here for $\mathrm{SNe}$ in our sample (Table 2) are AB magnitudes from PSF photometry on host-subtracted images.

Classification spectra of identified transients were obtained continuously by members of the PTF/iPTF collaboration, using different telescopes with apertures ranging from 2 to $10 \mathrm{~m}$, and their spectrographs. While some SNe were monitored extensively with spectroscopy, the majority of the SNe in our sample only have $\sim 1$ spectrum. The classification spectra used in this work are shown in Fig. A.1 and are listed in Table 3.

\section{The sample}

A total of 3018 confirmed SNe was found by PTF/iPTF in the years 2009-2017, all spectroscopically classified by the collaboration using template-matching software like SNID (Blondin $\&$ Tonry 2007), Gelato (Harutyunyan et al. 2008), or Superfit (Howell et al. 2005). Classifications of the PTF/iPTF SNe were done by a joint effort of members of the PTF/iPTF collaboration, involving discussions of ambiguous cases.

Among the $\mathrm{SNe}$ found and spectroscopically classified by PTF/iPTF during 2009-2017, a total of 111 are SNe IIn. These SNe IIn are located at declinations $-23^{\circ}<\delta<+75^{\circ}$ and in the redshift interval $0.0071<z<0.31$. The mean $z$ of the total SN IIn sample is 0.083 and the median $z$ is 0.07 . For our SN IIn sample, we made a selection based on criteria related to the availability of photometry for the rising and declining portions of the SN light-curves. Selection of our final sample of SNe IIn was conducted according to the following steps.

To obtain photometry of even quality from the P48 images, the PTFIDE pipeline was run on all available $R$ and $g$ images from $\mathrm{P} 48$ for the locations of the $111 \mathrm{SNe}$ in the initial sample to get forced template-subtracted photometry (using the procedure from Masci et al. 2015). For the light-curves thus obtained, we applied the constraint that a photometric upper limit must occur less than $40 \mathrm{~d}$ before the epoch of the PTF/iPTF discovery of the $\mathrm{SN}$ in order to allow a useful constraint on the rise time. This decreased the sample from 111 to 55 events. Including also inspection of the preliminary reductions of the P60 photometry, only SNe IIn with detections past $40 \mathrm{~d}$ after discovery were kept in the sample. After final refinement using the fully reduced P60 photometry, we were left with a sample of 42 SNe IIn with satisfactory light-curve coverage. The time intervals considered in the selection were in the observer's frame. Our SN sample selection process is summarised in Table 4. The classification spectra in the region around $\mathrm{H} \alpha$ for these $42 \mathrm{SNe}$ IIn are provided in Fig. A.1. Basic properties of the $\mathrm{SNe}$ in the sample are 
Table 3. $\log$ of the classification spectra for the $42 \mathrm{SNe}$ IIn in our sample.

\begin{tabular}{|c|c|c|c|c|}
\hline $\mathrm{SN}$ & $\begin{array}{l}\text { Epoch } \\
\text { (MJD) }\end{array}$ & $\begin{array}{c}\text { SN age, relative to } \\
\text { peak (days, rest frame) }\end{array}$ & Telescope & Instrument \\
\hline PTF09tm & 55037 & 8 & Lick & Kast \\
\hline PTF09uy & 55008 & -23 & Keck I & LRIS \\
\hline PTF09bcl & 55055 & 9 & WHT & ISIS \\
\hline PTF10cwl & 55270 & 4 & Keck I & LRIS \\
\hline PTF10cwx & 55273 & 1 & P200 & DBSP \\
\hline PTF10ewc & 55285 & -14 & WHT & ISIS \\
\hline PTF10fjh & 55300 & -8 & Gemini North & GMOS \\
\hline PTF10flx & 55299 & -6 & Gemini North & GMOS \\
\hline PTF10gvd & 55322 & 9 & Keck I & LRIS \\
\hline PTF10gvf & 55323 & -13 & Keck I & LRIS \\
\hline PTF10oug & 55396 & -30 & P200 & DBSP \\
\hline PTF10qwu & 55421 & - & P200 & DBSP \\
\hline PTF10tel & 55434 & -11 & Gemini North & GMOS \\
\hline PTF10tyd & 55443 & -23 & $\mathrm{P} 200$ & DBSP \\
\hline PTF10vag & 55454 & -10 & Lick & Kast \\
\hline PTF10weh & 55502 & -2 & Lick & Kast \\
\hline PTF10xgo & 55477 & -2 & P200 & DBSP \\
\hline PTF10abui & 55536 & -10 & P200 & DBSP \\
\hline PTF10achk & 55547 & -5 & $\mathrm{UH}$ & SNIFS \\
\hline PTF10acsq & 55574 & - & P200 & DBSP \\
\hline PTF11fzz & 55736 & -51 & P200 & DBSP \\
\hline PTF11mpg & 55833 & 6 & Keck II & DEIMOS \\
\hline PTF11oxu & 55872 & -37 & WHT & ISIS \\
\hline PTF11qnf & 55892 & -76 & Keck I & LRIS \\
\hline PTF11qqj & 55895 & -7 & P200 & DBSP \\
\hline PTF11rfr & 55916 & -30 & P200 & DBSP \\
\hline PTF11rlv & 55953 & 20 & Kitt Peak & RC Spec \\
\hline PTF12cxj & 56035 & -14 & Gemini North & GMOS \\
\hline PTF12frn & 56124 & 4 & Keck II & DEIMOS \\
\hline PTF12glz & 56123 & -58 & P200 & DBSP \\
\hline PTF12ksy & 56238 & -14 & P200 & DBSP \\
\hline iPTF13agz & 56442 & 26 & Apache Point & DIS \\
\hline iPTF13aki & 56395 & -7 & P200 & DBSP \\
\hline iPTF13asr & 56421 & 2 & Keck I & LRIS \\
\hline iPTF13cuf & 56545 & - & Keck II & DEIMOS \\
\hline iPTF14bcw & 56803 & -16 & Keck II & DEIMOS \\
\hline iPTF14bpa & 56832 & -28 & P200 & DBSP \\
\hline iPTF15aym & 57176 & -13 & NOT & ALFOSC \\
\hline iPTF15bky & 57196 & -8 & NOT & ALFOSC \\
\hline iPTF15blp & 57226 & 17 & P200 & DBSP \\
\hline iPTF15eqr & 57362 & -10 & Keck I & LRIS \\
\hline iPTF16fb & 57445 & 4 & P200 & DBSP \\
\hline
\end{tabular}

Notes. Epochs are given in MJD (=JD - 2400 000.5). The spectra were taken at epochs $-11 \pm 20 \mathrm{~d}(1 \sigma$ Gaussian spread $)$ relative to light-curve peak (Table 6). For our spectroscopy, we used the following telescopes (with spectrographs given in parenthesis): Apache Point $3.5 \mathrm{~m}$ (DIS, Dual Imaging Spectrograph), Gemini North $8.1 \mathrm{~m}$ (GMOS, Gemini Multi-Object Spectrograph), Keck I $10 \mathrm{~m}$ (LRIS, LowResolution Imaging Spectrometer), Keck II $10 \mathrm{~m}$ (DEIMOS, DEep Imaging Multi-Object Spectrograph), Kitt Peak $4 \mathrm{~m}$ (Ritchey-Chretien Spectrograph), Lick $3 \mathrm{~m}$ Shane telescope (Kast Double Spectrograph), Nordic Optical Telescope (NOT) $2.5 \mathrm{~m}$ (ALFOSC, Alhambra Faint Object Spectrograph and Camera), P200 $5.1 \mathrm{~m}$ Hale telescope (DBSP, Double Spectrograph), University of Hawaii (UH) $2.2 \mathrm{~m}$ (SNIFS, Supernova Integral Field Spectrograph) and William Herschel Telescope (WHT) $4.2 \mathrm{~m}$ (ISIS, Intermediate-dispersion Spectrograph and Imaging System). For SNe with age - when the spectrum was taken, no peak epoch was determined.

summarised in Table 5. In the cases when the PTF/iPTF discovery of a SN constituted an independent discovery of a SN already found (or later found) by others, a reference is given in Table 5. The 42 light-curves are shown in Fig. A.2. The SNe in the sample
Table 4. Selection of the SN IIn sample.

\begin{tabular}{ll}
\hline \hline Selection of SNe & Number of SNe \\
\hline All SNe found and classified by & 3018 \\
PTF/iPTF 2009-2017 & 692 \\
$\begin{array}{l}\text { Spectroscopically classified } \\
\text { SNe II }\end{array}$ & 111 \\
Spectroscopically classified & \\
SNe IIn & \\
SNe IIn having upper limits less & 55 \\
than 40 d before discovery & \\
SNe IIn also having detections & 42 \\
past 40 d after discovery & \\
\hline
\end{tabular}

Notes. The selection of SNe II(n) in each row is a subset of the SNe in the row above. The 692 "SNe II" include SNe IIP/L/b/n. The $40 \mathrm{~d}$ mentioned is in the observer's frame. The 15 SLSNe II found by PTF/iPTF are not included in the SNe II row and below.

are covered in BgRriz photometry (but not all SNe are covered in all photometric bands). The photometry presented here (Table 2) for the $42 \mathrm{SNe}$ is available via the VizieR database (Ochsenbein et al. 2000) and the classification spectra and the photometry is available via WISeREP (Yaron \& Gal-Yam 2012).

Table 5 shows that most of the SNe IIn in our sample have been mentioned in the literature, mostly in three sample studies (Ackermann et al. 2015; Ofek et al. 2014c,a) as well as in Circulars and other non-refereed sources. These sample studies mainly concerned themselves with $R$-band photometry (Ofek et al. $2014 \mathrm{c}$,a) or searches for $\gamma$-ray emission from the positions of the SNe (Ackermann et al. 2015), and did not publish full light-curves. PTF10tel $(=\mathrm{SN} 2010 \mathrm{mc})$ and its precursor activity is the topic of a paper by Ofek et al. (2013) and PTF12glz was studied by Soumagnac et al. (2019). Early-time spectra of PTF10gvf and PTF10tel were studied in the spectral sample paper by Khazov et al. (2016). For consistency, we will refer to the SNe by their PTF/iPTF names, even in the cases when International Astronomical Union names exist.

The superluminous SNe (SLSNe) IIn deserve special attention. A $M<-21 \mathrm{mag}$ limit has commonly been used to define SLSNe (Gal-Yam 2012, 2019) but it is not clear whether SNe IIn fulfilling this criterion constitute a separate population of events (e.g. Moriya et al. 2018; Gal-Yam 2019). During the time when the SNe IIn in our sample were found and classified, the $M<$ -21 mag limit was in common use and the 111 SNe Type IIn that we used to construct our final sample are therefore typically fainter at peak than this limit.

Not all SLSNe Type II display narrow Balmer lines in their spectra (Inserra et al. 2018). In the full PTF/iPTF catalogue of spectroscopically classified SNe a total of 15 SLSNe II are listed. For 7 of the $\mathrm{SNe}$, the spectra are either noisy or contaminated by host galaxy emission lines and make a SLSN Type IIn classification difficult. The spectrum of PTF12gwu has weak Balmer lines (Perley et al. 2016), but possibly broad. The remaining 7 of the SLSN Type II display a narrow $\mathrm{H} \alpha$ emission component ostensibly making them SLSNe Type IIn. Applying our criteria (see above) requiring a maximum gap of $40 \mathrm{~d}$ between discovery epoch and last pre-discovery upper limit, leave 6 SLSNe Type IIn. Also requiring photometry past 40 days after discovery leaves 5 SLSNe IIn: PTF10heh, PTF12mkp, PTF12mue, iPTF13duv and iPTF13dol. The PTF/iPTF photometry shows that these 5 SLSNe Type IIn had light-curve peaks 
A. Nyholm et al.: Type IIn SN light-curve properties

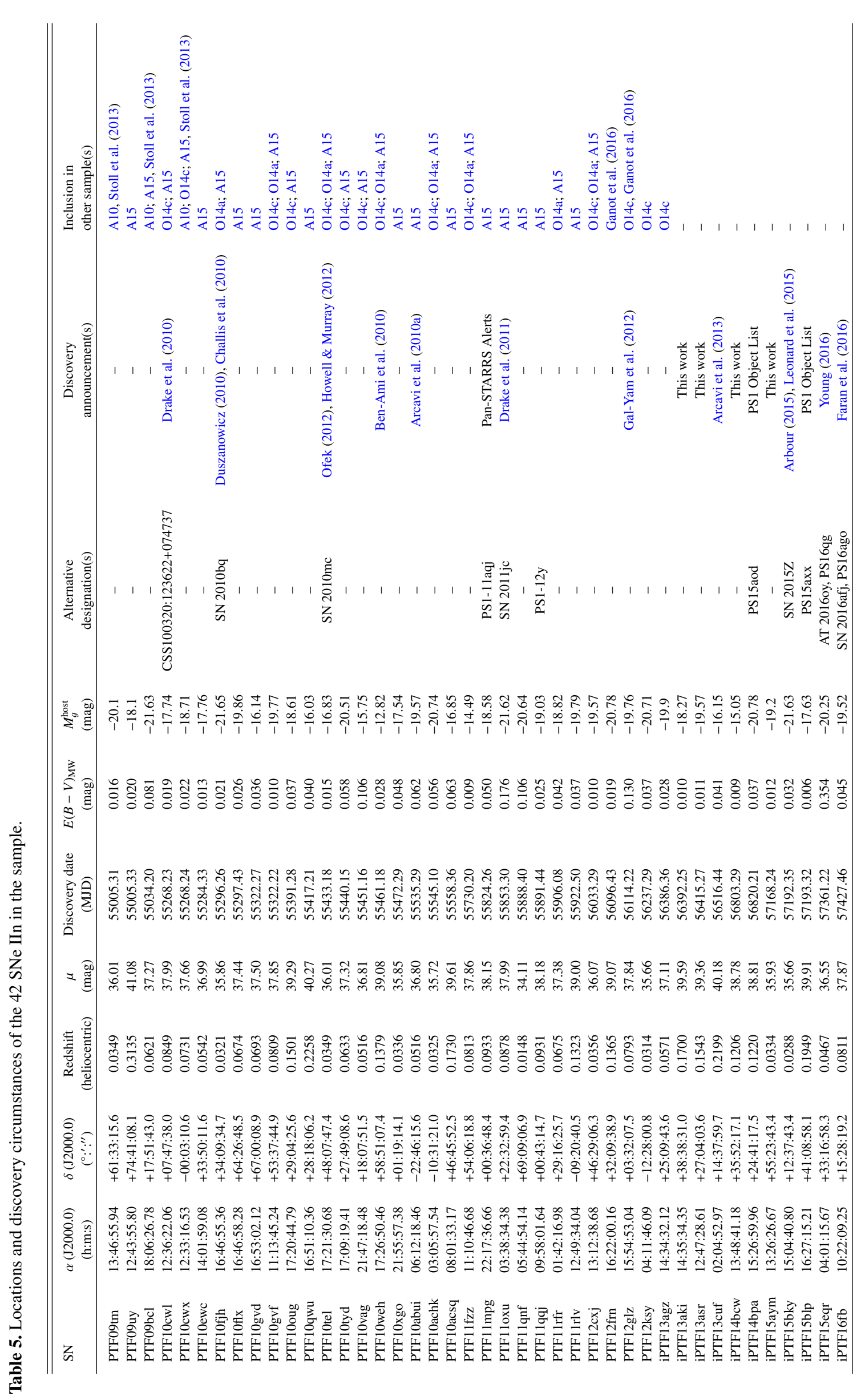

ชิ

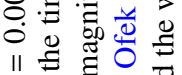

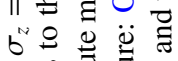

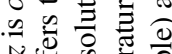

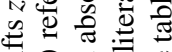

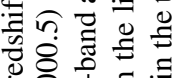

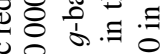

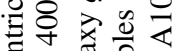

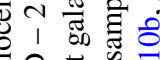

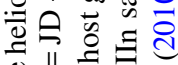

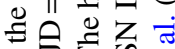

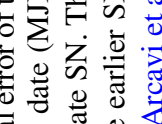

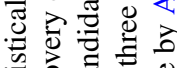

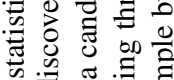

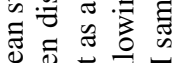

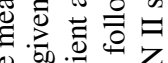

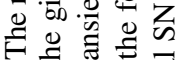
的踏 这 离

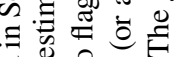

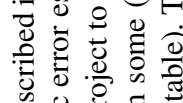
这.

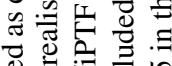
政

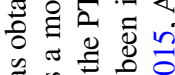

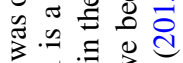

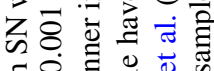
尊 论 훈해

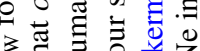

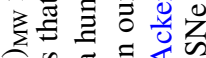
5 政

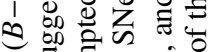

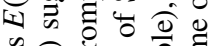
象

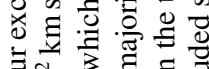
言 항

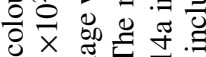
争的

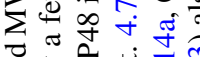

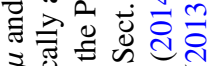
政

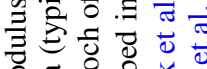

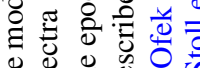
o

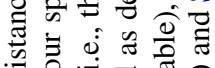
의 青范

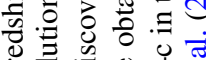
政 흥

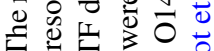

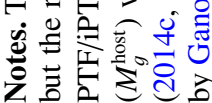




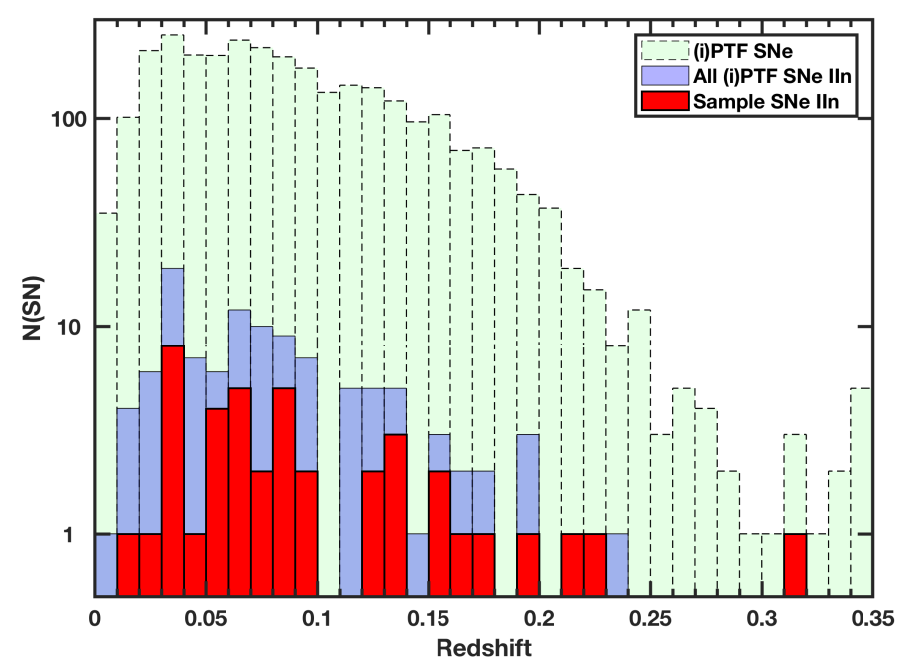

Fig. 1. Histogram of redshifts for the $42 \mathrm{SNe}$ IIn included in our sample, shown in red. The redshifts of the full PTF/iPTF sample of $111 \mathrm{SNe}$ IIn is shown in blue. For comparison, the redshifts of the full PTF/iPTF yield of spectroscopically classified $\mathrm{SNe}$ (for $z<0.35$ ) is shown in green.

at -21 mag. The photometry for two of these (PTF12mue and iPTF13duv) is too sparse to allow us to further characterise their light-curves.

We therefore used the sample of $111 \mathrm{PTF} / \mathrm{iPTF}$ SNe IIn (with its inherent use of the $M<-21$ mag SLSN criterion) when selecting our SN IIn sample. For discussing whether the SLSNe Type IIn constitute a separate population of SNe, we include the additional 3 SLSNe IIn (PTF10heh, PTF12mkp and PPTF13 $\mathrm{dol}^{1}$ ) in the peak-magnitude distribution and in our luminosity function study in Sect. 4.6. For comparison purposes, we also include PTF12mkp when discussing the durationluminosity phase space in Sect. 4.8. PTF10heh and PTF12mkp are already presented in the literature (Perley et al. 2016). A study dedicated to SLSNe II from PTF is ongoing (Leloudas et al., in prep.).

In this paper, our SN distance estimates are based on the SN redshifts, assuming a $\Lambda \mathrm{CDM}$ cosmology and using the cosmological parameters $H_{0}=70.0 \mathrm{~km} \mathrm{~s}^{-1} \mathrm{Mpc}^{-1}, \Omega_{\Lambda}=0.721$, and $\Omega_{\mathrm{M}}=0.279$ (Hinshaw et al. 2013). We use a method based on the NASA/IPAC Extragalactic Database (NED) routine (following Mould et al. 2000a,b) to compensate for Virgo, Great Attractor, and Shapley cluster infall in our distance estimates. The redshifts of the sample SNe were measured by fitting Gaussian profiles to the $\mathrm{H} \alpha$ emission lines of their spectra. The histogram of the redshifts is shown in Fig. 1.

Milky Way (MW) extinction values from Schlafly \& Finkbeiner (2011) were obtained via NED. Extinctions for different photometric bands were calculated using the extinction function by Ofek (2014) based on Cardelli et al. (1989) and assuming an absorption-to-reddening ratio $R_{V}=3.1$. If $\mathrm{SN}$ spectra showed a clear NaID absorption doublet, host-galaxy extinction was computed using Taubenberger et al. (2006, their Eq. (1)). Such indications of host-galaxy extinction were seen only in the spectra of PTF09tm $(E(B-V)=0.16 \mathrm{mag})$, PTF11qnf $(E(B-V)=0.72 \mathrm{mag})$, and PTF12frn $(E(B-V)=$ $0.57 \mathrm{mag})$. We remind the reader that given the quality and resolution of our classification spectra, we are not very sensitive to

\footnotetext{
1 iPTF13dol was found by iPTF on 2013 September 29 at $\alpha=$
} $22^{\mathrm{h}} 22^{\mathrm{m}} 07.27, \delta=12^{\circ} 30^{\prime} 39^{\prime \prime} \cdot 9(\mathrm{~J} 2000.0)$ and $z=0.225$. this method. The effects of these assumptions on our results are discussed in Sect. 5.2.

\section{Analysis}

In the following analysis we characterise the main light-curve parameters, that is peak absolute magnitude and its epoch, lightcurve decline rates, and rise times. We also investigate possible correlations between these properties. We describe the optical colours and the host-galaxy properties. Finally, we compare our observations to simple models in order to derive information on the CSM and on the SN progenitor scenarios. In our analysis, we apply corrections to the following SNe: for PTF10acsq, a correction (found by interpolation on P60 r-band detections) of 0.07 mag was added to the P48 $R$-band; by similar interpolation on P60 $g$-band detections, for iPTF15aym 0.72 mag was added to the P48 $g$-band, and for iPTF15eqr 0.19 mag was added (by interpolation on $\mathrm{P} 60 r$ ) to the P48 $R$-band.

\subsection{Peak magnitudes and peak times}

The differences in cadence, photometric errors, as well as intrinsic light-curve shapes make the use of a single analytical template difficult for characterising the light-curves. Instead, we use cubic smoothing splines (CSS; de Boor 2001) to fit the lightcurves and to find peak-magnitude values and times of peak for our sample SNe. Before fitting, we convert the photometry from $\mathrm{AB}$ magnitudes to flux densities (using the convert_flux function by Ofek 2014).

The fitting of a CSS to a set of data is determined by the smoothing parameter $s$, where the fit approaches a least-squares (LSQ) fit of a straight line as $s \rightarrow 0$, whereas the fit approaches a cubic-spline interpolant between each data point as $s \rightarrow 1$. To objectively choose an $s$ value for each light curve, we proceed in the following way.

For a grid of $45 s$ values with decadal spacing in the interval $10^{-5} \leq s \leq 0.9$, a CSS fit was made for each $s$ value. For each CSS fit, the number of degrees of freedom (d.o.f.) was computed as the number of observations minus the number of parameters of the CSS fit, and then used to calculate the $\chi^{2}$ values of each CSS fit (per d.o.f., i.e., the reduced $\chi^{2}$ values, $\chi_{\text {d.o.f. }}^{2}$ ).

For selecting the $s$ value, we use the Akaike Information Criterion (AIC; see, e.g. Sect. 2 of Davis et al. 2007) as our statistic. The AIC punishes models of high orders (i.e., "overparametrised" models). As we desire a CSS fit capturing the large-scale behaviour of the light-curve (while not being sensitive to smaller fluctuations), we choose to consider values of $s<0.1$. We generally select the smoothing parameter $s$ giving the smallest AIC value for $s<0.1$. If no minimum of the AIC value is found for $s<0.1$, an $s$ value is selected corresponding to the AIC value $3 \sigma$ above the mean AIC value for the interval $0.01<s<0.1$.

Armed with our selected $s$ parameter for each SN, Monte Carlo (MC) experiments were done to estimate the uncertainties of the maximum flux density and the peak epoch. For each MC run, pseudo data points were generated for each epoch in the light curve, drawn from Gaussian distributions around the original data points (from within the $1 \sigma$ photometric error bars). For the $s$ value found above, CSS fits were made to each such pseudo light curve, and maximum flux density and peak epoch were determined for each of them. The mean values of the highest flux density and its epoch are used as the final values, and their standard deviations are used as their $1 \sigma$ uncertainties. This process 

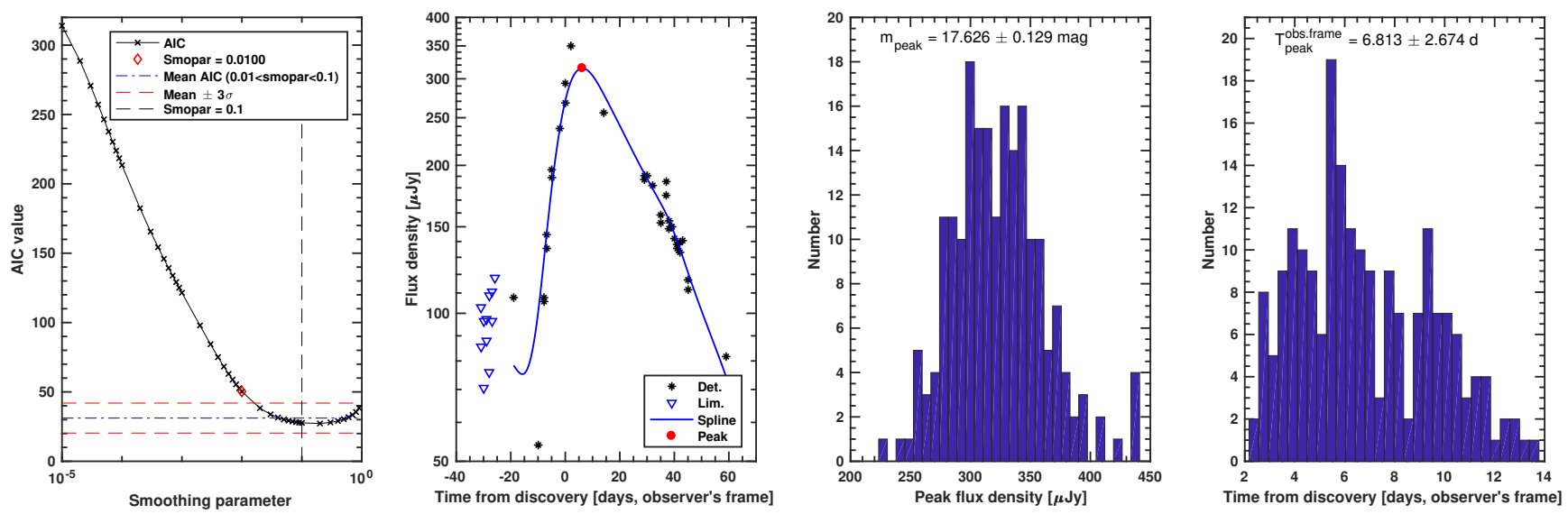

Fig. 2. Left to right: AIC values as a function of the smoothing parameter of the attempted spline fits, light-curve (in flux density), peak flux density (Monte Carlo histogram), and peak time (Monte Carlo histogram) example for PTF10achk.

is illustrated in Fig. 2. The CSS fits are shown in Fig. A.3, along with the measured peak of each light curve. The peak magnitudes are presented in Table 6, where the absolute magnitudes are corrected for extinction. In our later analysis of decline rates and rise times, the $\mathrm{SNe}$ with peak time error $>10 \mathrm{~d}$ (marked with red crosses in Fig. A.3) are excluded.

To evaluate if one or more groupings can be distinguished among the $\mathrm{SNe}$ when considering a given property (such as peak absolute magnitude), we use Gaussian mixture models (GMM) following Papadogiannakis et al. (2019). The method works by applying an increasing number of Gaussian distributions to the data, using the AIC for each step to evaluate the significance of each combination of Gaussians. The best combination of Gaussians is the one giving the lowest value of AIC. Given our small sample we also tried the Bayesian information criterion (BIC), which is considered to rule out overparametrised models in a way stronger than the AIC (Liddle 2004). Following Sollerman et al. (2009, their Sect. 4.1) we consider models separated by $\Delta \mathrm{IC} \geq 6$ from the surrounding models as significant. Owing to our relatively small sample size (42 $\mathrm{SNe}$ ), we limit ourselves to examining 1 to 3 combined Gaussians.

Applying GMM to the distribution of peak absolute magnitudes, the AIC would prefer a combination of 3 Gaussians describing the distribution. The BIC favours a single Gaussian describing the peak absolute magnitude distribution. For simplicity, we consider this single Gaussian (Fig. 3) in our analysis.

The observed peak-magnitude distribution for the sample is shown in Fig. 3 along with the best-fit Gaussian distributions. The $g$-band photometry was used when measuring four of the $\mathrm{SNe}$ (see Table 6). Of our sample SNe IIn, the peak magnitudes within $1 \sigma$ from the mean peak magnitudes range between -17.8 and -20.0 mag (a factor of 8 in luminosity).

Comparing these peak-magnitude ranges to known SNe IIn in the literature (central panel in Fig. 3), we notice that many well-studied SNe IIn peak within the -17.8 to -20.0 mag range as found above. In the bottom panel of Fig. 3, we show the $39 \mathrm{SNe}$ with determined peak epoch and mark the -17.8 to -20.0 mag range with horizontal dash-dot lines. The large total range of peak absolute magnitudes for the sample $(\sim 5 \mathrm{mag})$ is clearly shown. Kiewe et al. (2012) and Taddia et al. (2013) found the peak absolute magnitudes of the SNe IIn they studied to be in the interval $-19 \lesssim M_{B} \lesssim-17 \mathrm{mag}$, consistent with the findings of other authors (Richardson et al. 2002, 2014; Li et al. 2011) and with our result.

\subsection{Light-curve decline rates}

Among the first events classified as SNe IIn it was noticed that some faded slowly compared to other CC SNe. SN $1988 Z$ (Stathakis \& Sadler 1991; Aretxaga et al. 1999) declined at $\sim 0.2 \mathrm{mag}(100 \mathrm{~d})^{-1}$ in the $R$ band past $100 \mathrm{~d}$ after peak brightness. SN 1998S (Liu et al. 2000; Fassia et al. 2000) instead had a fast initial decline of $\sim 2.5 \mathrm{mag}(100 \mathrm{~d})^{-1}$ in the $R$ band. Sample measurements of SN IIn decline rates have been presented by Taddia et al. (2013) and Kiewe et al. (2012).

In the MC experiments with the CSS fits (Sect. 4.1), the flux density values at $50,100,150,200$, and 250 days past peak were determined (if occurring within the time range where the $\mathrm{SN}$ was detected) for calculation of decline rates in these time intervals. This is done for the photometry obtained in the BgRri bands, in the cases where the coverage exceed $50 \mathrm{~d}$ (rest frame) after the peak times given in Table 6 . The decline rates for $0-50 \mathrm{~d}$ for the $R / \mathrm{r} / \mathrm{g}$ bands are provided in Table 6 . The distribution of the decline rates between 0 and $50 \mathrm{~d}$ post-peak (for the $27 \mathrm{SNe}$ possible to measure) is shown as a histogram in the top panel of Fig. 4.

A GMM test (Sect. 4.1) gives inconclusive results, with the BIC significantly suggesting one Gaussian distribution of decline rates. Such a single distribution has $0.027 \pm$ $0.016 \mathrm{mag} \mathrm{d}^{-1}$ and is overplotted in the top panel of Fig. 4. However, AIC suggests two Gaussian distributions, but with weak significance: one of slow decliners $\left(0.013 \pm 0.006 \mathrm{mag} \mathrm{d}^{-1}\right)$ and one of fast decliners $\left(0.040 \pm 0.010 \mathrm{mag} \mathrm{d}^{-1}\right)$. No K-corrections were applied when measuring these rates. Overplotting these decline rates on the actual SN light-curves (scaled to match at peak brightness) in the bottom panel of Fig. 4 visualises this result. The central panel shows that most of our fast-declining SNe IIn are actually declining faster than SN 1998S (often considered as a prototypical fast decliner).

To allow a straightforward comparison between decline rates of $\mathrm{SNe}$ at different redshifts, in Fig. 5 we plot the decline rates as a function of the effective rest wavelength (for the filter used and the redshift of the SN in question, as in for example González-Gaitán et al. 2015; Taddia et al. 2015b). In the 0-50 d panel, we see the lack of slow decliners in the bluer bands. This is similar to what is seen in other SN types (e.g. SNe IIP; Valenti et al. 2016) where also the bluer bands exhibit 


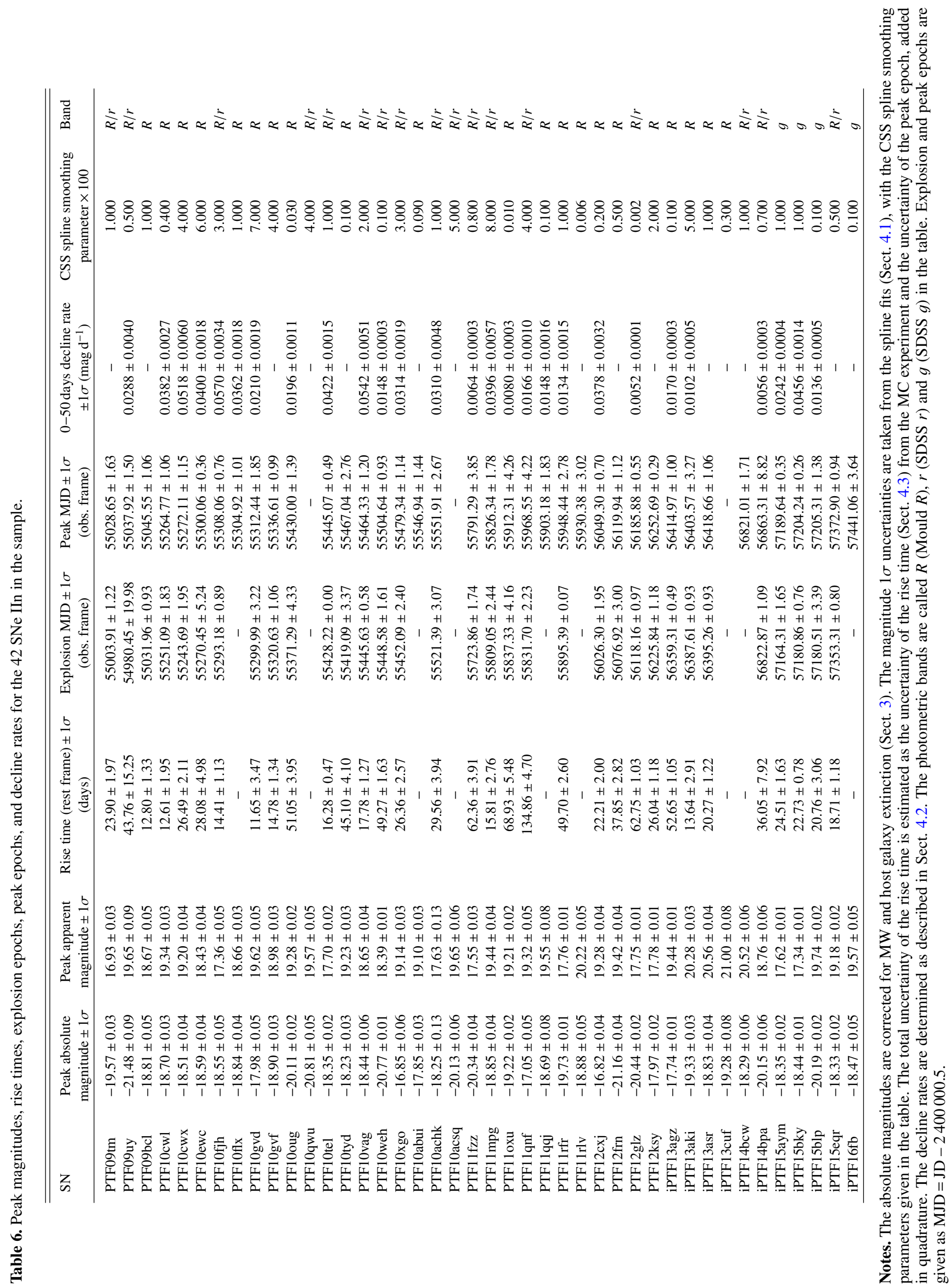



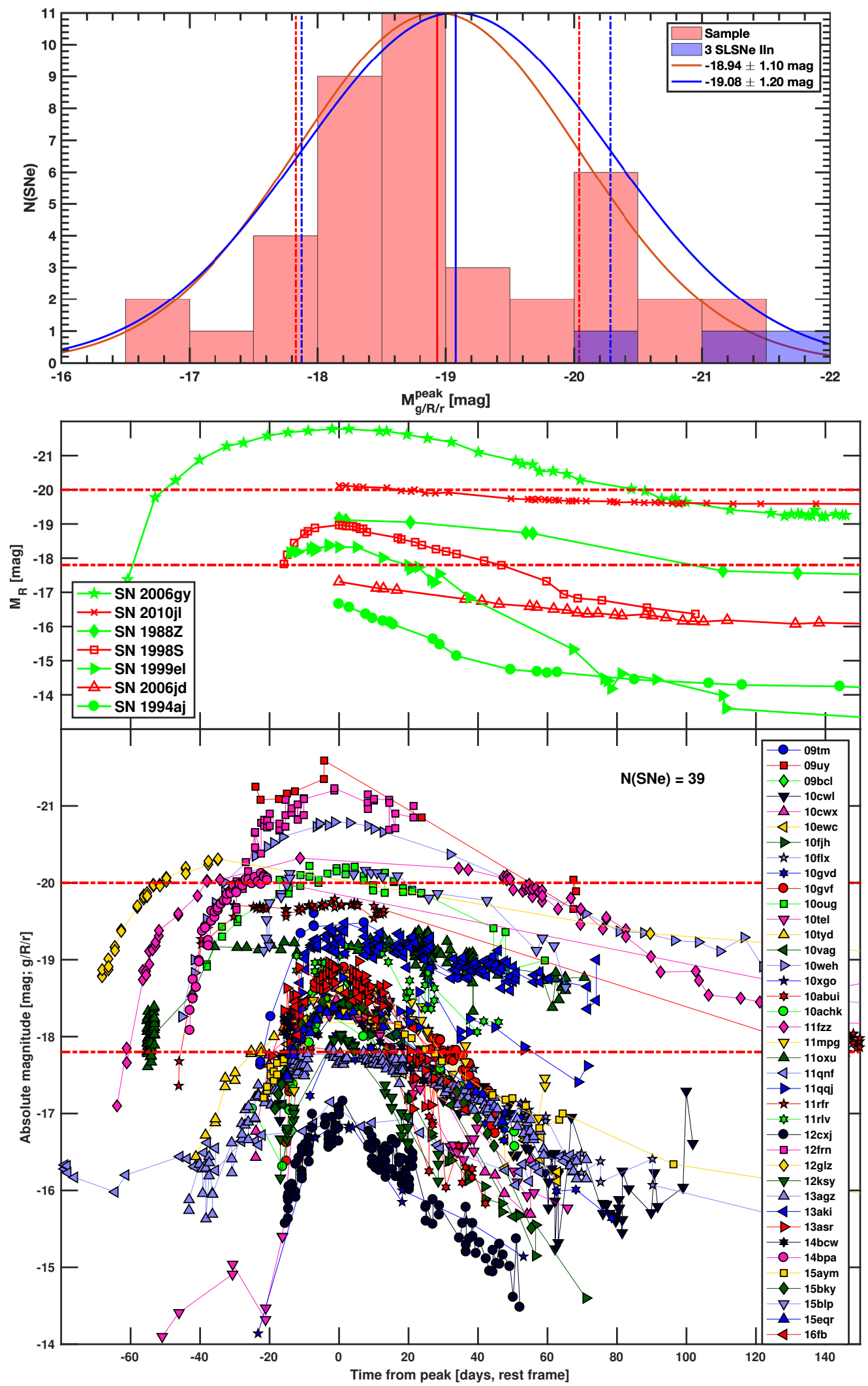

Fig. 3. Top panel: histogram for the peak absolute magnitudes of our $42+3 \mathrm{SNe}$ IIn, overplotted with the best-fit Gaussian distributions as identified with GMM. The Gaussian describing the distribution for the sample of $42 \mathrm{SNe}$ is shown in red, the Gaussian for the sample $42 \mathrm{SNe}$ IIn +3 SLSNe IIn is shown in blue. Dash-dot lines show the $1 \sigma$ values for the respective Gaussians. Central panel: some well-studied SNe IIn from the literature, showing photometry of SN 1988Z (Aretxaga et al. 1999), SN 1994aj (Benetti et al. 1998), SN 1998S (Liu et al. 2000), SN 1999el (Di Carlo et al. 2002), SN 2006gy (Smith et al. 2007), SN 2006jd (Stritzinger et al. 2012), and SN 2010j1 (Fransson et al. 2014), with -17.8 mag and -20.0 mag ( $1 \sigma$ values from the sample distribution) shown as dash-dot red lines. Bottom panel: absolute magnitudes of the $\mathrm{N}(\mathrm{SNe})=39$ SNe IIn with determined peak epochs as a function of time relative to light-curve peak (with sample $1 \sigma$ values shown as dashed red lines). No $\mathrm{K}$-corrections were applied in these plots. 

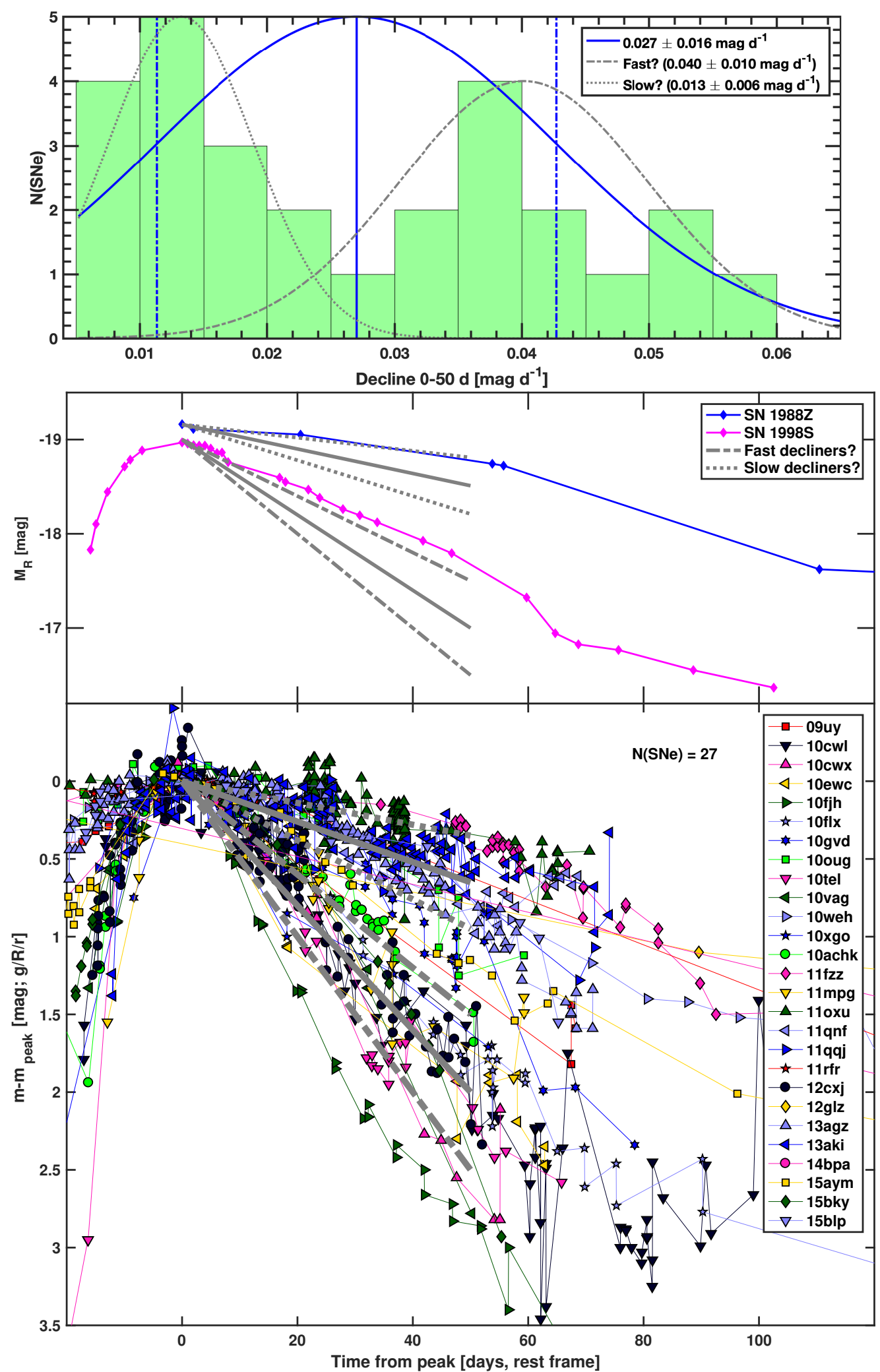

Fig. 4. Top panel: histogram for the decline rates between 0 and $50 \mathrm{~d}$ after peak brightness for 27 of our SNe IIn. A single Gaussian (identified using GMM with BIC) describing the whole population is shown in blue, but our SNe IIn might possibly divide into fast (grey dash-dot lines) and slow (grey dotted lines) decliners, with slopes $0.040 \pm 0.010 \mathrm{mag} \mathrm{d}^{-1}$ and $0.013 \pm 0.006 \mathrm{mag} \mathrm{d}^{-1}$, respectively (suggested by GMM with AIC). The respective mean decline rates are shown as solid grey lines. Central panel: two possible clusters of SNe IIn (slow and fast decliners) compared to the prototypical slow and fast decliners from the literature (SN 1988Z, Aretxaga et al. 1999; SN 1998S, Liu et al. 2000). Bottom panel: light-curves of the $\mathrm{N}(\mathrm{SNe})=27$ of our SNe IIn with measured decline rates (between 0 and $50 \mathrm{~d}$ ), scaled to match at peak. The two different decline rates are overplotted. Fast (grey dash-dot lines) and slow (grey dotted lines) decliners are shown, with the respective mean decline rates marked with solid grey lines. 


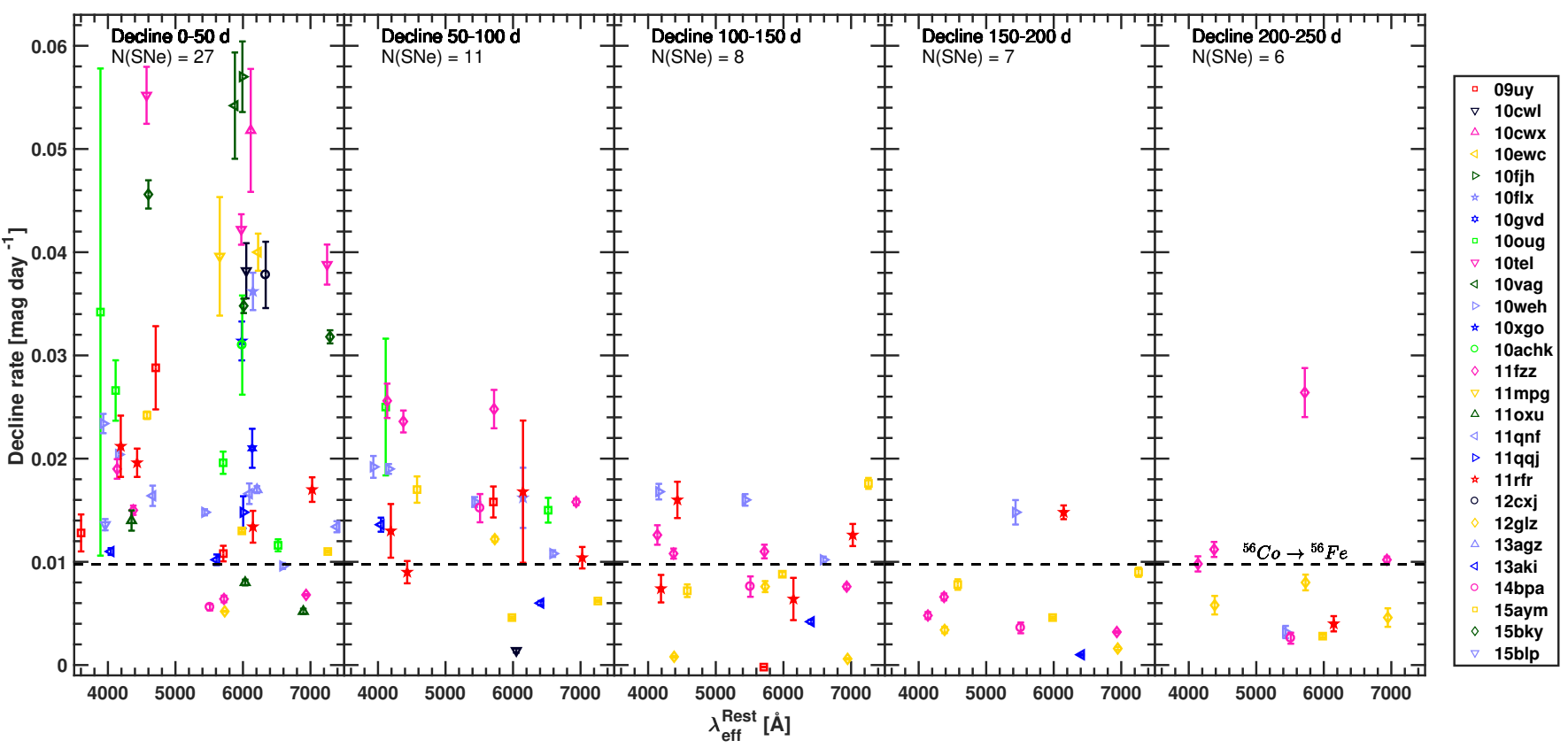

Fig. 5. Decline rates 0-250 rest-frame days (past the peak time given in Table 6) of 27 of the SNe in our sample. For reference, the decay rate $0.0097 \mathrm{mag} \mathrm{d}^{-1}$ of ${ }^{56} \mathrm{Co} \rightarrow{ }^{56} \mathrm{Fe}$ is shown as a dashed black line. The number of unique $\mathrm{SNe}$ in each panel is given as $\mathrm{N}(\mathrm{SNe})$.

the fastest decline rates. For the panels showing times $>50 \mathrm{~d}$, the number of $\mathrm{SNe}$ gets smaller. This likely involves observational bias, since these $\mathrm{SNe}$ are becoming too faint for us to monitor. Some SNe IIn (like SN 2005ip; see Stritzinger et al. 2012) had an initial rapid decline, followed by a long-lasting $(>100 \mathrm{~d})$ fainter plateau. Such faint and late plateaus would be missed in this study for many of our SNe, owing to their large distances. In Fig. 5, the decline rate plotted for PTF11fzz in the 200-250 d panel is illusive and caused by the photometric filter effect discussed in Sect. 5.4.

Although SNe IIn are assumed to primarily be driven by circumstellar interaction (CSI), we can use the decline rates found here to evaluate if radioactive decay can be a possible energy source in some of the $\mathrm{SNe}$ in our sample. For CC SNe such as $\mathrm{SNe}$ IIP, the rate of decay for ${ }^{56} \mathrm{Co} \rightarrow{ }^{56} \mathrm{Fe}$ determine the lightcurve slope around $\sim 200 \mathrm{~d}$ after light-curve peak. Figure 5 shows that some SNe around $200 \mathrm{~d}$ after peak have decline rates consistent with radioactive decay. From Fig. A.2, it can be seen that these $\mathrm{SNe}$ have absolute magnitudes of $M \approx-17$ at this time. Calculations using Nadyozhin (1994, his Eq. (19)) indicate that the original ${ }^{56} \mathrm{Ni}$ mass necessary to drive such a light-curve is $\sim 1 M_{\odot}$, which is at least an order of magnitude more than for normal SNe IIP (e.g. Hamuy 2003; Rubin et al. 2016; Anderson 2019). Most SNe in our sample indeed decline more slowly than the radioactive decay, and we consider radioactive decay as an unrealistic mechanism to explain the late-time luminosities and decay rates seen in our sample of SNe IIn.

\subsection{Light-curve rise times}

By fitting a function to the rising portion of a SN light curve, the starting time of the light-curve can be constrained, given the assumption that the unseen early portion of the rise is smooth and that the fitted function is consistent with the pre-discovery upper limits in the photometry. Functions commonly used for such fits are power-law functions (González-Gaitán et al. 2015; Ofek et al. 2014c; Cowen et al. 2010) or exponential functions (González-Gaitán et al. 2015; Ofek et al. 2014c; Bazin et al.
2009), which may have little physical motivation but allow quantification of the SN light-curve rise.

In our work, we are primarily interested in finding a consistent way to measure the rise times of our sample SNe IIn, without characterising the shape of the light-curve rise in detail for each SN. For this purpose, we use a power-law light-curve template based on a well-studied, nearby $\mathrm{SN}$ in our sample.

Based on the procedure in Sect. 4.1, the SNe with peak time error $>10$ days (PTF10qwu, PTF10acsq, iPTF13cuf) are excluded also in the rise time analysis. The available pre-peak photometry does not allow a reliable measurement of the rise time if the difference in magnitude covered during the rise is too small, or if the photometry is taken during a too short time interval. For the magnitude interval $(\Delta m)$ and time interval $(\Delta t)$ covered by $n$ pre-peak detections, we therefor exclude any SN having $\Delta m<0.60 \mathrm{mag}$ and $n / \Delta t>0.40 \mathrm{~d}^{-1}$. This removes PTF10abui, PTF10flx, PTF11rlv, PTF11qqj, iPTF14bcw and iPTF16fb from the rise time analysis.

To make a rising light-curve template for rise-time measurements, we considered SNe from our sample with $z<0.075$, having the first detection within $4 \mathrm{~d}$ of the last upper limit (in the observer's frame) and this first detection having $m_{R}>21 \mathrm{mag}$. At $z=0.075$, this means $M_{R} \gtrsim-16.5 \mathrm{mag}$. This gives the rising light-curve template candidates PTF10cwx, PTF10tel, PTF10tyd, PTF11qnf, and iPTF13agz. We choose PTF10tyd as our template owing to it having the lowest $\chi_{\text {d.o.f. }}^{2}$ for a fitted $t^{2}$ power law. A least-squares fit to the PTF10tyd rising light-curve (flux density scale) gives the rest-frame template with the rise time $\Delta t=47.5 \mathrm{~d}$ (Fig. 6) for the power-law model

$L(t)=L_{\text {peak }}\left[1-\left(\frac{t-t_{\text {peak }}}{\Delta t}\right)^{2}\right]$

where $L_{\text {peak }}$ is the peak luminosity from the fit, $\Delta t$ is the time from 0 to peak luminosity, and $t_{\text {peak }}$ is the time of the peak luminosity (Ofek et al. 2014c, their Eq. (5)). This template is stretched, scaled, and shifted in order to fit the other SNe in our sample (see Fig. A.4). For demonstration, in Fig. 6 we also show 


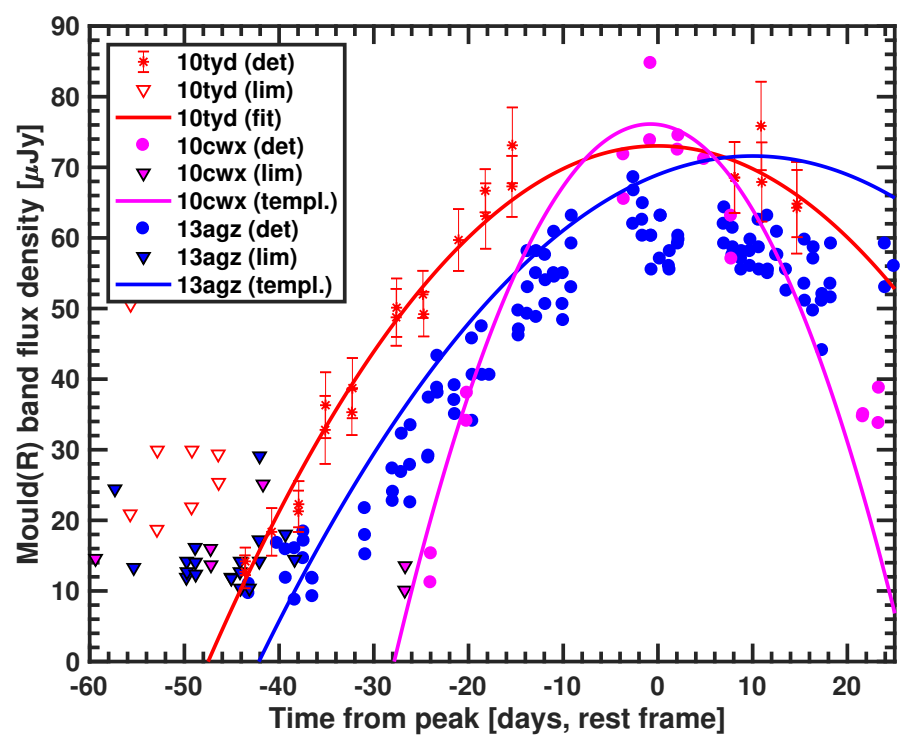

Fig. 6. Light-curve ( $R$-band) of PTF10tyd, with weighted fit of the $\propto t^{2}$ template shown along with the rise template candidates PTF10cwx and iPTF13agz. Scale, stretch, and shift parameters as found in Sect. 4.3 are applied.

the PTF10tyd template stretched, scaled, and shifted to the lightcurves of template candidates PTF10cwx and iPTF13agz.

In Fig. A.4 we have converted pre-peak AB magnitudes of the SN to flux density as in Sect. 4.1 and normalised the flux density values to peak brightness. We then stretch the light-curve data points in time by applying a multiplicative factor, scale their position in flux density (additively), and shift their position in time (additively) to minimise the distance (in a LSQ sense) of the light-curve data points to the power-law template. When this is done, the derived stretch value is used to compute the rise time of the input SN (as $t_{\text {rise }}=\Delta t_{\mathrm{PTF} 10 t \mathrm{tyd}} /$ stretch). A MC experiment is done to generate new light-curve data points within the light-curve error bars. The light-curve stretch, scale, and shift operations are then repeated to estimate the uncertainty of the stretch value.

For the cases where the obtained $\propto t^{2}$ template fit ends up inconsistent (i.e., brighter) than an upper limit observed before the first detection, the time of that upper limit is assumed as an approximation of the "start of rise" epoch. In such cases, as an estimate of the uncertainty we assume the time between the upper limit and the first detection. For the special case of PTF10tel (Ofek et al. 2013), with its detected photometric activity before the start of rise, we adopt the explosion epoch given by Ofek et al. (2013) as the "start of rise" epoch. The derived rise times (in the rest frame) are presented in Table 6.

We plot the histogram of the rise times in the top panel of Fig. 7. The SN IIn population is characterised by a large range of rise times, and a GMM test (Sect. 4.1) shows a significant division (for both AIC and BIC) into two clusters: fast-rising $\mathrm{SNe}$ IIn with rise times of $20 \pm 6 \mathrm{~d}$ (magenta lines in Fig. 7) and slow-rising SNe IIn with rise times of $50 \pm 11 \mathrm{~d}$ (blue lines). PTF11qnf, where we found a rise time to peak of $135 \mathrm{~d}$ (Table 6) and a faint $\left(M_{r} \approx-17 \mathrm{mag}\right)$ peak, is an outlier not included in this GMM rise-time analysis. PTF11qnf might have been an SN impostor (Sect. 5.3).

The literature about SNe IIn already showed examples of fast-rising SNe IIn like SN 1998S and slow-rising SNe IIn like the SLSN Type IIn SN 2006gy. These two SNe are displayed in the central panel of Fig. 7 and the rising portions of their lightcurves are compared to the two rise-time ranges we found in our sample. We overplot all our SNe IIn with measured rise times in the bottom panel of Fig. 7, to further illustrate the variety of rise-time values and the relatively similar light-curve shapes. We particularly note the erratic light-curve of PTF11qnf as well as the precursor activity prior to the start of rise for PTF10tel (as analysed by Ofek et al. 2013 and compared to SN 2009ip by, for example, Pastorello et al. 2018; Smith et al. 2014). In Sect. 5 we further discuss these events.

\subsection{Correlations between light-curve properties}

Having measured the main light-curve-shape parameters, we proceed to investigate if these quantities are somehow correlated in our sample. Ofek et al. (2014b) proposes a correlation between rise time and peak luminosity, based on the assumption of the SN shock breaking out in the CSM. This was studied observationally by Ofek et al. (2014c), inferring a possible correlation. From analytical models, Moriya \& Maeda (2014) instead indicate that no strong dependence on rise time should be seen for the peak luminosity (shock breakout does not have to occur in the CSM of all SNe IIn). In Fig. 8 we use the peak luminosities obtained from absolute $R / r$-band magnitudes $M_{r}$ with

$L\left(M_{r}\right)=\left(3.04 \times 10^{35}\right) \times 10^{\left(-0.4 M_{r}\right)} \mathrm{erg} \mathrm{s}^{-1}$

based on solar bolometric values and, following Ofek et al. (2014a) and Nyholm et al. (2017), neglecting bolometric corrections. A unweighted Spearman correlation test for the $\mathrm{SNe}$ plotted in Fig. 8 gives the correlation coefficient $p=0.23$, corresponding to a significance of $\sim 1.19 \sigma$. We report the significance for a given correlation coefficient $p$ as $\sqrt{2} \times \operatorname{erf}^{-1}(1-p)$, using a two-sided tail of the normal distribution. Excluding the possible SN impostor PTF11qnf (Sect. 5.3) gives $p=0.08(\sim 1.8 \sigma)$ ). If a correlation between rise time and peak luminosity exists at all, it is weak.

Using a bootstrap technique, Ofek et al. (2014c) claimed a significance of $\sim 2.5 \sigma$ for their sample of $15 \mathrm{SNe}$ IIn (12 of which are in our sample). With our method, we find a significance of $\sim 1.75 \sigma$ for this Ofek et al. (2014c) sample. Finding a similar significance with our larger sample (32 SNe; excluding PTF11qnf) also suggests that the correlation, if any, is weak.

In Fig. 9 (left panel) we find a correlation with $p=0.0037$ $(\sim 2.9 \sigma)$ for decline rate versus rise time. This shows that slowly rising $\mathrm{SNe}$ IIn are also slow decliners, and fast risers are fast decliners. The empty upper-right corner of the decline rate versus rise time plot of Fig. 9 shows that we do not see any $\mathrm{SNe}$ having both a slow rise and a fast decline. For decline rate versus peak absolute magnitude (Fig. 9, right panel) we obtain $p=0.0065(\sim 2.7 \sigma)$, suggesting that the more luminous SNe IIn decline slower. This is somewhat comparable to the Phillips relation (Pskovskii 1977; Phillips 1993) between peak magnitude and decline rate for SNe Ia, but less tight in the SN IIn case. These correlations for SNe IIn can be discerned in the bottom panel of Fig. 3.

As a control, we investigate the presence of bias by looking for correlations between rise or decline rates and the peak apparent magnitudes of the SNe IIn. Such a correlation might indicate bias affecting the correlations discussed above. The decline rate versus peak apparent magnitude gives $p=0.28(\sim 1.1 \sigma)$. The rise time versus peak apparent magnitude gives $p=0.52$ 

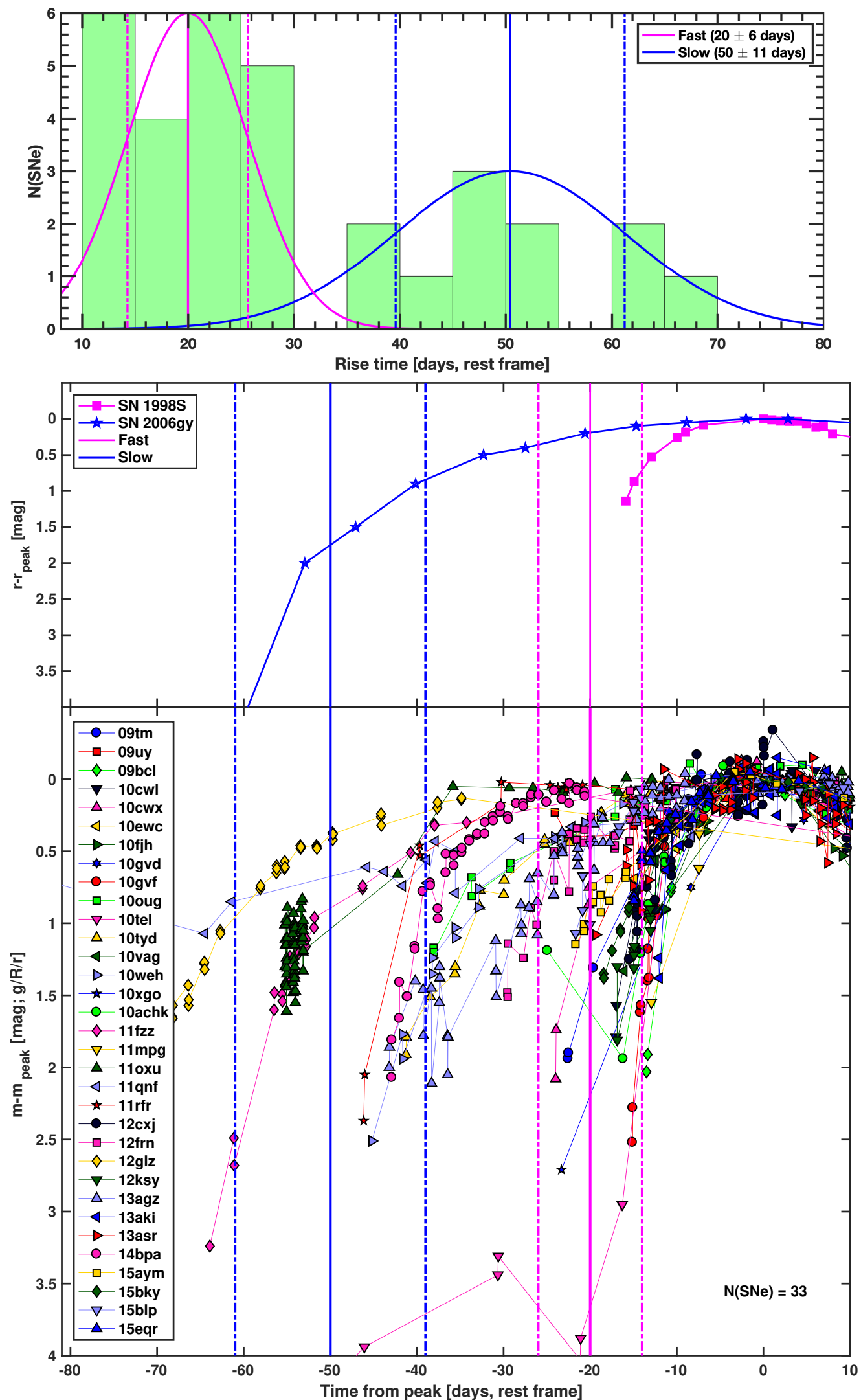

Fig. 7. Top panel: histogram for the rise times for 32 of our SNe IIn. Our SNe IIn are divided into fast (magenta lines) and slow (blue lines) risers, with rise times $20 \pm 6 \mathrm{~d}$ and $50 \pm 11 \mathrm{~d}$, respectively (as identified with GMM). Dashed lines show $1 \sigma$ of the Gaussians. For reasons discussed in Sect. 5.3, we excluded PTF11qnf from the GMM analysis. Central panel: rise times of the two clusters of SNe IIn (slow and fast risers) compared to two well-known slow and fast risers from the literature (SN 2006gy, Smith et al. 2007; SN 1998S, Liu et al. 2000), with the rise-time ranges from the top panel overplotted. Bottom panel: light-curves of all the $\mathrm{N}(\mathrm{SNe})=33 \mathrm{SNe}$ in our sample with well-determined peak times matched at peak brightness (here, we include PTF11qnf for completeness). 


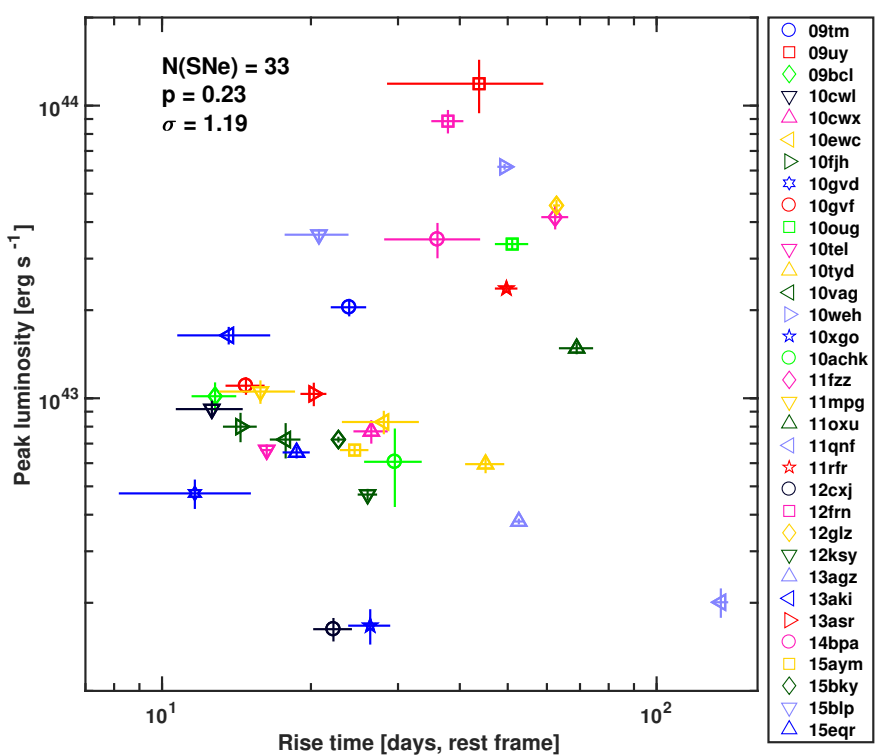

Fig. 8. Rise times and peak luminosities for 33 of the $\mathrm{SNe}$ in the sample, using measurements described in Sect. 4.3, following the investigation by Ofek et al. (2014c). The photometric band used for each $\mathrm{SN}$ is given in Table 6. The Spearman correlation coefficient $p$, the corresponding significance $\sigma$ and the number of $\mathrm{SNe}, \mathrm{N}(\mathrm{SNe})$, are shown.

$(\sim 0.65 \sigma)$. The absence of a correlation in both cases suggests that the correlations with absolute magnitude could be real.

\subsection{Colour evolution}

Only a small number of SN IIn sample studies have considered the colour evolution of the SNe IIn in a collective fashion. Compilations of colour evolution of SNe IIn were presented by Taddia et al. (2013) and de la Rosa et al. (2016), based on their respective samples. In Fig. 10, we present the $g-r$ and $g-i$ colour evolution for 22 of our SNe IIn with respect to the light-curve peak epochs. The magnitudes used were corrected for extinction as described in Sect. 3.

For comparison, we also plot the $g-r$ and $g-i$ colours of the slowly evolving SNe IIn 2005ip and 2006jd (photometry from Stritzinger et al. 2012) and the rapidly evolving SNe IIn 2006aa and 2008fq (photometry from Taddia et al. 2013). The colour curves for these four events from the literature have been computed using the MW and host extinctions and peak epochs given in the respective papers. The evolution of the $g-i$ colour is rather monotonic for the sample as a whole, albeit with some spread. The SNe 2006aa and 2008fq have $g-i$ colour evolution encompassing most of our sample SNe, while SNe IIn with the slower $g-i$ colour evolution of SNe 2005ip and 2006jd are not seen among our 22 plotted SNe. The $g-i$ colour evolving towards the red for the majority of the SNe reflects their decreasing blackbody continuum temperatures. The $g-r$ colour evolution is likely affected by the evolution of the $\mathrm{H} \alpha$ emission line (with a line centre within the SDSS $r$ filter for $z \lesssim 0.065$, where 7 of the 22 SNe in Fig. 10 are located).

The small spread in colours suggests that the colour corrections applied (Sect. 3) are without large errors. Exceptions are the $g-r$ colour of iPTF15eqr and the $g-i$ colour of PTF11qnf. In the case of PTF11qnf, a possible SN impostor, its colour index and its host $E(B-V)$ is discussed in Sect. 5.3.

Our analysis of rise and decline behaviour (Sect. 4.4) as well as the luminosity function (Sect. 4.6) is based on $R / r$ - band photometry as far as possible, but when the $R / r$-band is not available, we used the $g$-band instead. This is the case for four of our SNe (Table 6). Via linear interpolation between epochs surrounding time of peak, we find the colours of each $\mathrm{SN}$ at peak. The $g-r$ colour index at peak of the SNe IIn is $g-r=0.06 \pm 0.21 \mathrm{mag}$ and $g-i=0.13 \pm 0.46 \mathrm{mag}$. This is consistent with $g-r \approx 0$ mag and $g-i \approx 0$ mag around peak brightness, and it indicates that our use of $g$-band for four $\mathrm{SNe}$ in our analysis should not affect our conclusions.

\subsection{Luminosity function}

The observed luminosity function (see Fig. 3, top panel) of the $\mathrm{SNe}$ in our sample is affected by Malmquist bias (Malmquist 1922). This means that SNe intrinsically luminous at peak (thus easier to detect in a magnitude-limited survey like PTF/iPTF) are over-represented compared to the intrinsically less luminous SNe. To reduce the impact of the Malmquist bias on our estimated luminosity function, we use the bootstrap method described by Richardson et al. (2014) as implemented by Taddia et al. (2019).

Figure 11 (top panel) shows the peak absolute magnitude as a function of distance modulus $(\mu)$ for our 42 sample $\mathrm{SNe}$. PTF12cxj with $M_{r}^{\max }=-16.82 \mathrm{mag}$ is the intrinsically faintest $\mathrm{SN}$ in the sample and the dashed diagonal line represents the typical limiting magnitude of the PTF/iPTF survey under good conditions, $m_{R} \approx 21 \mathrm{mag}$. This indicates that our sample satisfactorily represents the SN IIn population up to $\mu \approx 37.8 \mathrm{mag}$ $(z \approx 0.08)$. This is consistent with the sample redshift distribution shown in Fig. 1. In this discussion, all magnitudes are assumed to be in the $r$ band (cf. Sect. 4.5).

We consider the peak-magnitude distribution observed at $\mu<37.8 \mathrm{mag}$ as intrinsic in the intervals $-17.8<M_{r}^{\max }<$ $-16.8 \mathrm{mag}$ and $-18.8<M_{r}^{\max }<-17.8 \mathrm{mag}$, respectively, and randomly generate the peak magnitudes of the missing $\mathrm{SNe}$ with $-17.8<M_{r}^{\max }<-16.8 \mathrm{mag}$ in the interval $37.8<\mu<38.8 \mathrm{mag}$ based on these intrinsic distributions. The same random generation of missing $\mathrm{SNe}$ is repeated for the more distant $\mu$ intervals and are shown as empty red diamond symbols in Fig. 11 (top panel). The observed luminosity function of the SN sample is $M_{\text {peak }}=-18.96 \pm 1.11$ mag whereas the Malmquist-biascompensated luminosity function has $M_{\text {peak }}=-18.60 \pm 1.25$ mag $(1 \sigma$ spread $)$ as shown in Fig. 11.

The sample of 42 SNe IIn was selected from a body of SNe IIn all deemed to have $M_{\text {peak }}>-21$ mag (Sect. 3) and thus not being SLSNe according to the traditional (Gal-Yam 2012) criterion. In the study by Richardson et al. (2014), SNe IIn with $M_{\text {peak }}<-21 \mathrm{mag}$ were also included, so to facilitate a comparison of results, we repeated the above Malmquist-bias correction for the $45 \mathrm{SNe}$ IIn encompassing the main sample of $42 \mathrm{SNe}$ IIn and the 3 SLSNe IIn (PTF10heh, PTF12mkp, and iPTF13dol) introduced in Sect. 3. The observed luminosity function of this extended SN sample has $M_{\text {peak }}=-19.10 \pm$ $1.21 \mathrm{mag}$ whereas its bias-corrected luminosity function has $M_{\text {peak }}=-18.73 \pm 1.35$ mag. Richardson et al. (2014) found a bias-corrected distribution of $M_{\text {peak }}=-18.53 \pm 1.36 \mathrm{mag}$, similar to our result.

Richardson et al. (2014) applied a K-correction based on spectra of SN 1995G (slowly declining; Pastorello et al. 2002) and SN 1998S (rapidly declining; Fassia et al. 2001) in their calculations. When we include our K-corrections and their uncertainties (using Eqs. (B.1) and (B.2); for any $z>0.31$, we assume the K-correction for $z=0.31$ ) the Malmquist-bias-compensated luminosity function becomes $M_{\text {peak }}=-18.72 \pm 1.32 \mathrm{mag}$. Here, 
A. Nyholm et al.: Type IIn SN light-curve properties
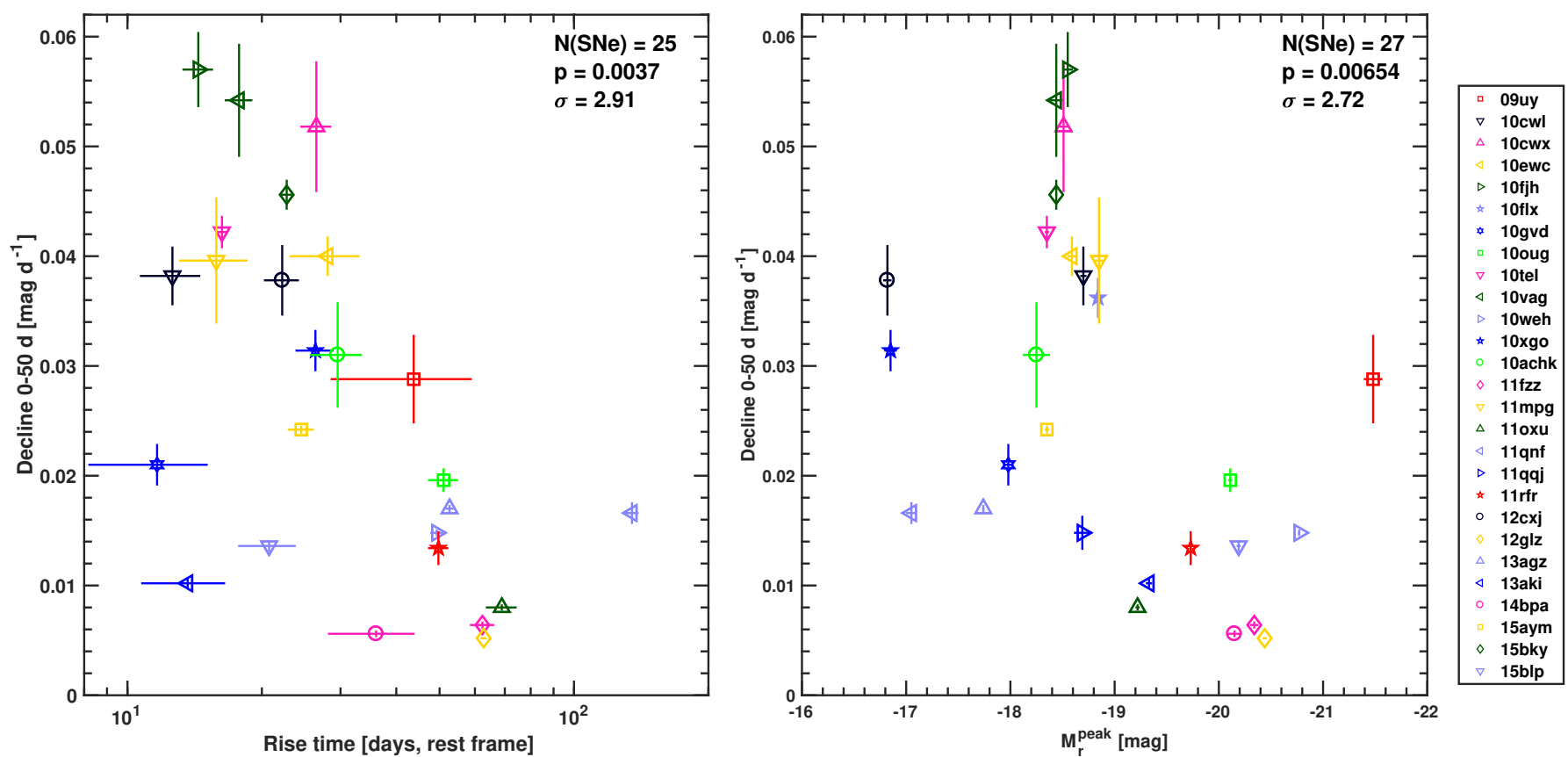

Fig. 9. Examination of possible correlations between decline rates versus rise times and peak absolute magnitudes, respectively, of the sample SNe (Sect. 4.4). Photometric bands are as specified in Table 6. In each panel the Spearman correlation coefficient $p$, the corresponding significance $\sigma$ and the number of $\mathrm{SNe}, \mathrm{N}(\mathrm{SNe})$, are shown.

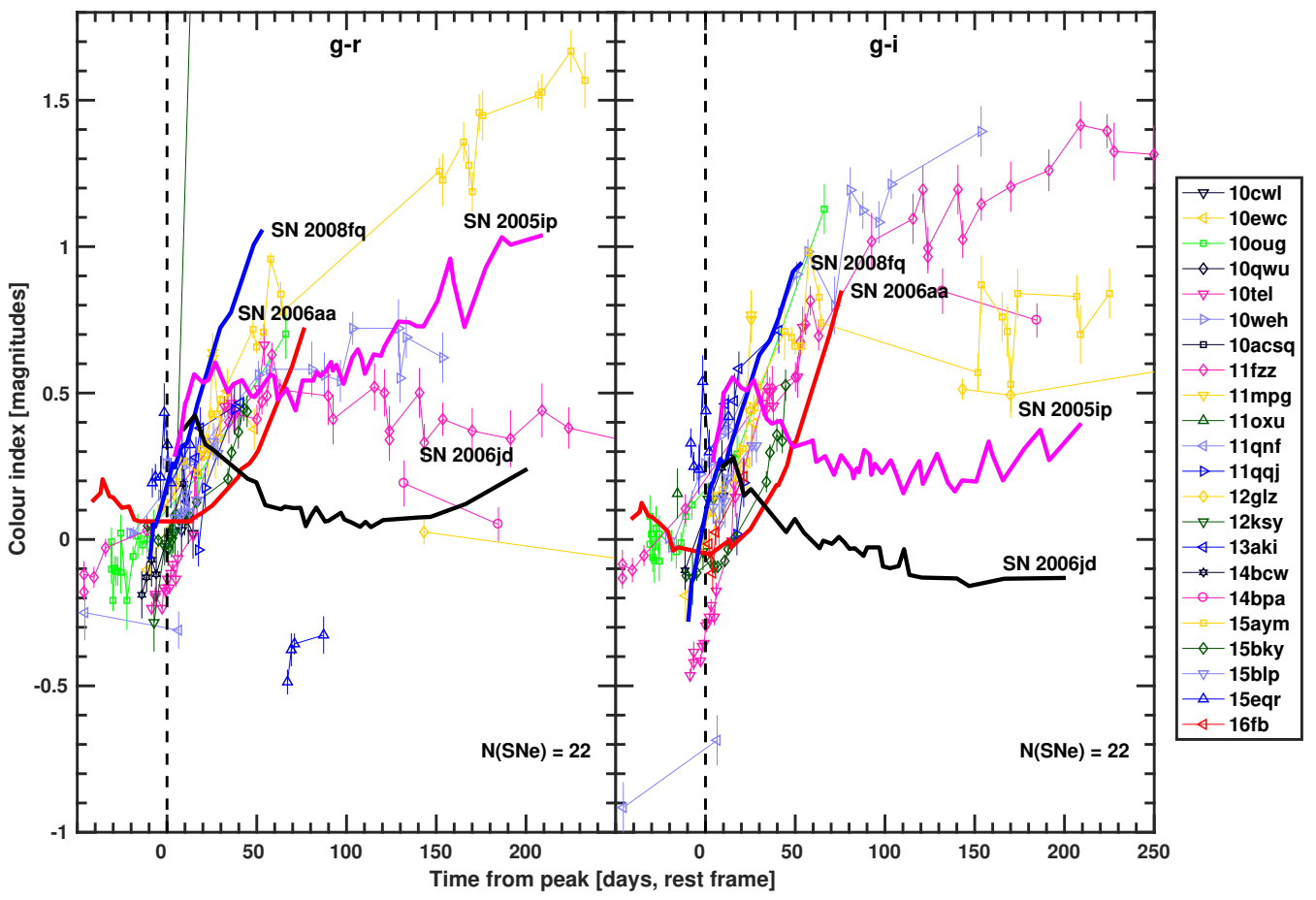

Fig. 10. Evolution of $g-r$ and $g-i$ colours for $22 \mathrm{SNe}$ in the sample. Host and MW extinction have been removed (Sect. 3). The vertical black dashed line shows the light-curve peak epoch (Sect. 4.1). Lines are drawn between data points to guide the eye. For clarity, colour data points with uncertainties $>0.1 \mathrm{mag}$ are not shown. For comparison, SNe IIn 2005ip, 2006jd (Stritzinger et al. 2012), 2006aa, and 2008fq (Taddia et al. 2013) have their colour curves shown with bold lines.

we assume that all the SNe were observed in the $R / r$-band. For the extended SN sample (including the 3 SLSNe IIn) and including K-corrections, we find the Malmquist-bias-corrected luminosity function $M_{\text {peak }}=-19.18 \pm 1.32 \mathrm{mag}$. The mean values of the derived $\mathrm{K}$-corrected distributions are more luminous than the ones without K-correction by $0.12 \mathrm{mag}$ and $0.45 \mathrm{mag}$, respectively, but consistent within $1 \sigma$.

\subsection{Host galaxies}

The host galaxies of our sample of $\mathrm{SNe}$ can be expected to have a wide range of absolute magnitudes, since the $\mathrm{SNe}$ were found in an untargeted search not giving priority to any special galaxy type. In earlier SN searches, nearby galaxies with lively star formation were targeted to increase the chance of finding $\mathrm{SNe}$ 

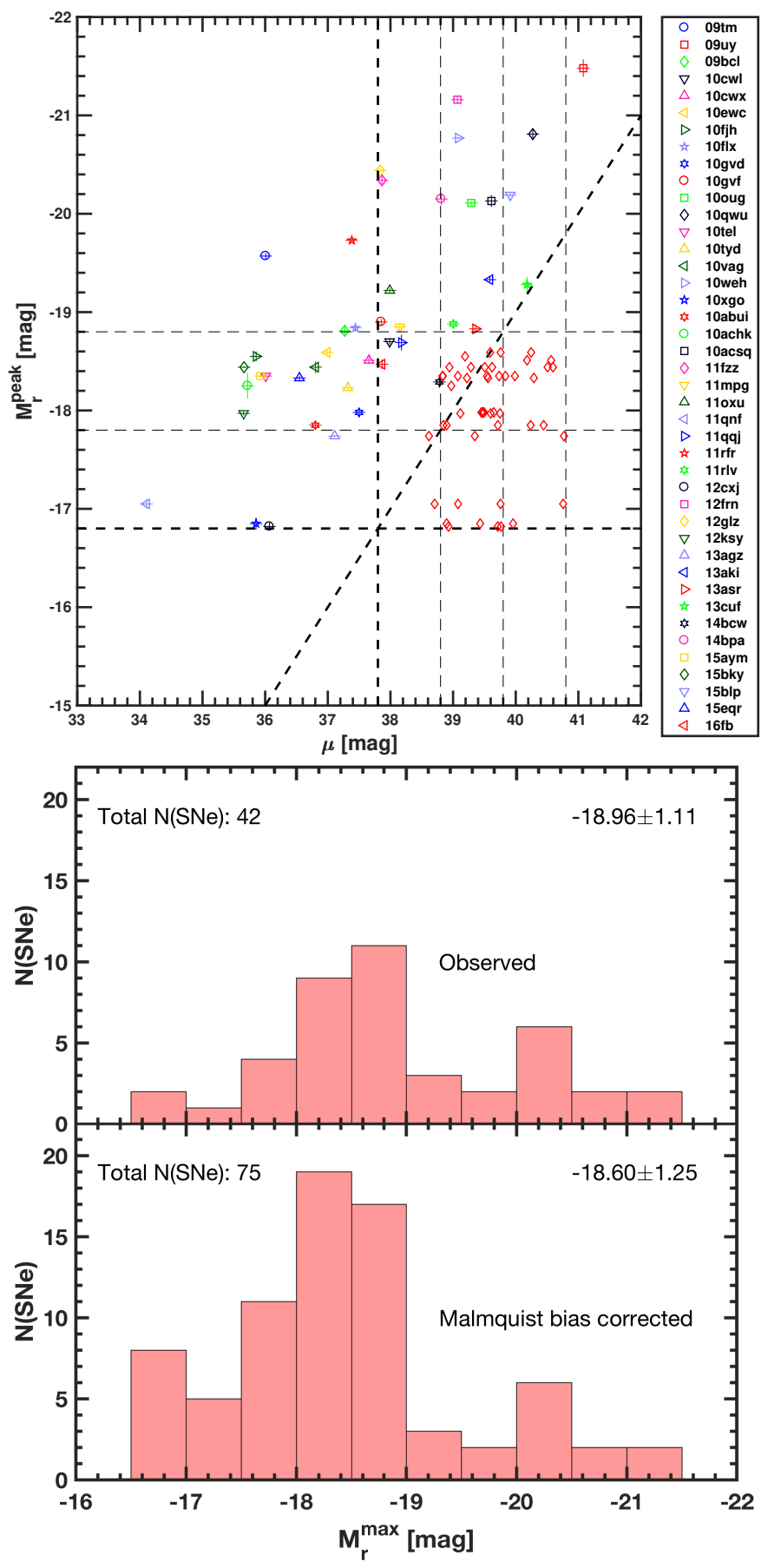

Fig. 11. Top panel: peak absolute magnitude $\left(M_{r}^{\text {peak }}\right)$ as a function of distance modulus $(\mu)$ of the $42 \mathrm{SNe}$ in the sample, shown with the randomly generated SNe IIn (empty red diamonds) introduced to compensate for Malmquist bias. The limiting magnitude $m=21$ typical for $\mathrm{PTF} / \mathrm{iPTF}$ under favourable conditions is shown as a diagonal, dashed line. Vertical and horizontal dashed lines show $M_{r}^{\text {peak }}$ and $\mu$ intervals described in Sect. 4.6. Bottom panel: histograms of the observed and bias-compensated luminosity distributions of the SNe IIn at peak brightness. Bias compensation follows Richardson et al. (2014) and Taddia et al. (2019). K-corrections are not taken into account in the results plotted here, but are studied in Sect. 4.6.

(Anderson \& Soto 2013). The sample of SNe IIn whose environments were studied by Taddia et al. (2015a) has a bias towards hosts being large spiral galaxies. Here, we examine whether our sample $\mathrm{SNe}$ are hosted by galaxies covering a different range of absolute magnitudes than the hosts in the Taddia et al. (2015a) sample.

From a study of the properties of PTF/iPTF SN host galaxies (Schulze et al., in prep.), we collected $g$-band absolute magnitudes of the host galaxies of our 42 sample SNe. Schulze et al. obtained the total flux of a galaxy in the different SDSS filters via a curve-of-growth analysis. For galaxies outside the SDSS field, Pan-STARRS images were used instead. In the case of PTF09uy and PTF10qwu the measurements in Perley et al. (2016) were used. The absolute magnitudes were obtained by modelling their spectral energy distributions with Le PHARE (Arnouts et al. 1999; Ilbert et al. 2006). The host absolute magnitudes (largely consistent with Petrosian magnitudes, Petrosian 1976) are given in Table 5.

We limited our comparison to the Taddia et al. (2015a) SN IIn sample hosts also in the SDSS field, obtaining their Petrosian magnitudes under the PhotoTag section of the SDSS DR $15^{2}$ Object Explorer web pages, considering galaxies with petroMagErr_g $<0.2 \mathrm{mag}$. This gave 23 galaxies. We used Schlafly \& Finkbeiner (2011) to get the foreground extinctions and the redshifts from Taddia et al. (2015a) with our infallcompensation method (Sect. 3) to calculate absolute magnitudes. For the redshifts $(\lesssim 0.02)$ of these literature $\mathrm{SN}$ hosts, K-corrections are generally small $(\lessgtr 0.2 \mathrm{mag}$; Chilingarian et al. 2010) and can be neglected. The cumulative distribution functions of the host galaxy $g$-band absolute magnitudes are shown in Fig. 12. For completeness, we note that two SNe IIn from the Taddia et al. (2015a) sample ( $\mathrm{SNe}$ 1997ab and 2007va) were hosted by dwarf galaxies; these galaxies did not pass our petroMagErr_g requirement.

The 42 sample SN host galaxies have $-21.6<M_{g}<$ $-12.8 \mathrm{mag}$ (8.8 mag interval). For the $23 \mathrm{SN}$ host galaxies from the Taddia et al. (2015a) sample, the absolute magnitudes are $-21.6<M_{g}<-17.0$ (4.6 mag interval). The host galaxies of the SNe from our untargeted sample thus cover a range of absolute magnitudes about 4 mag wider (towards the fainter end) than the SN host galaxies from the targeted searches for which we have reliable SDSS photometry, also having a different distribution. Schulze et al. (in prep.) shows that the mass distribution of the SN IIn hosts in our sample is consistent with the mass distribution of the hosts of the entire PTF/iPTF yield of SNe IIn. Schulze et al. (in prep.) also show it to be consistent with the distribution of masses for PTF/iPTF hosts of SNe Ib, Ic, IIb and II. These galaxy mass distributions are also consistent with mass distributions of CANDELS (Cosmic Assembly Near-IR Deep Extragalactic Legacy Survey, Grogin et al. 2011) galaxies weighted by star-formation rate (Schulze et al. 2018, their Fig. 12). This confirms that SNe IIn arise from massive stars and indicates that there is nothing peculiar with the SN IIn hosts in the sample.

The luminosity-metallicity relation by Tremonti et al. (2004, their Fig. 5) shows that the spread in $M_{\mathrm{g}}$ for our sample hosts in Fig. 12 corresponds to a wide range of global galaxy metallicities, from above solar metallicity (up to approximately $12+$ $\log \left(\frac{\mathrm{O}}{\mathrm{H}}\right) \approx 9$ on the scale of Kobulnicky \& Kewley 2004), to $12+\log \left(\frac{\mathrm{O}}{\mathrm{H}}\right) \lesssim 8$ (i.e., subsolar) for the fainter end of our distribution $\left(M_{g} \gtrsim-16 \mathrm{mag}\right)$. Earlier work on SN IIn environments (Kelly \& Kirshner 2012; Habergham et al. 2014; Taddia et al. 2015a; Anderson et al. 2015; Galbany et al. 2018; Kuncarayakti et al. 2018) suggests that studies of global properties of host galaxies are insufficient when detailed conclusions are to be drawn about $\mathrm{SNe}$ IIn and their progenitor

2 http://skyserver.sdss.org/dr15/en/tools/ 


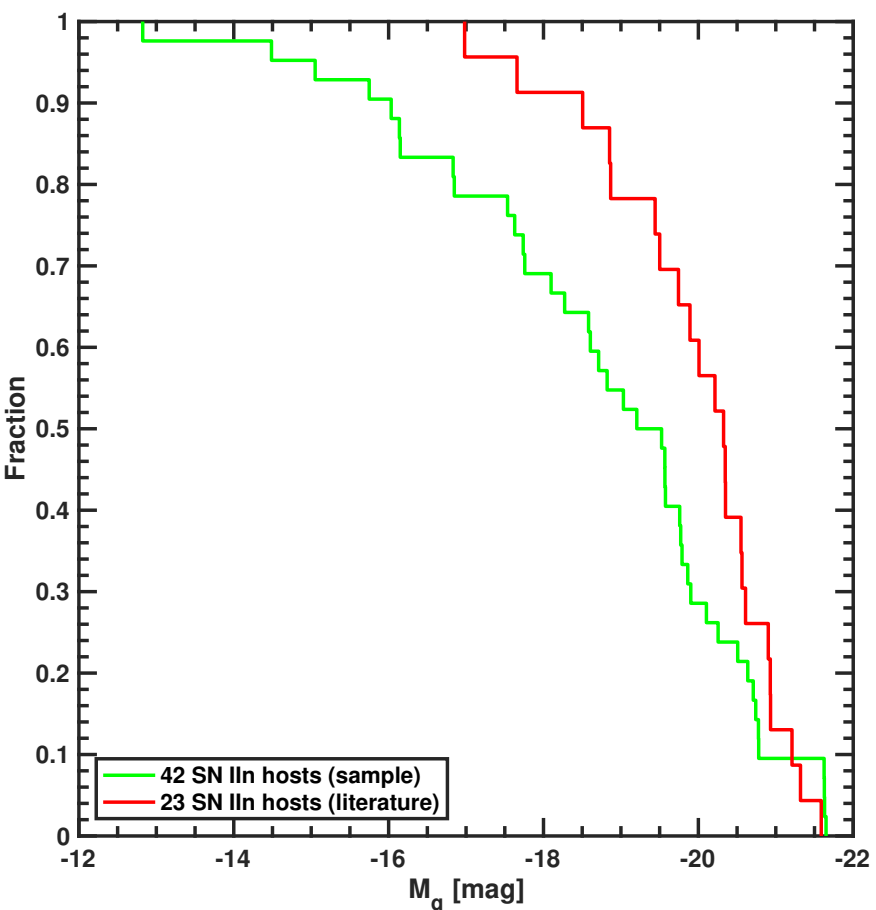

Fig. 12. Cumulative distribution plot of host-galaxy SDSS/PanSTARRS $g$-band absolute magnitudes (from Schulze et al., in prep.) for the 42 hosts of our sample SNe and for 23 SN IIn hosts in the SDSS field from the Taddia et al. (2015a) sample.

channels ${ }^{3}$, but it is clear that an untargeted search like the one used to build our SN sample has the potential to probe a more representative part of the galaxy population.

\subsection{Interpretation using models}

As discussed in Sect. 4.4, Ofek et al. (2014b) claim the possible existence of a correlation between the rise time and the peak luminosity of SNe IIn, assuming that the CSM close to the progenitor star is dense enough to allow shock breakout (SBO) to happen in the CSM. In Sect. 4.4, we saw that such a correlation hardly exists.

As an alternative to studying the relations between peak absolute magnitude and rise time or decline rates (Sect. 4.4), the relation between peak absolute magnitude and overall duration can also be considered. The location of the $\mathrm{SNe}$ in this duration-luminosity phase space (DLPS) can give insights into the mechanisms of the SNe. Using our spline fits (Sect. 4.1) we can measure the duration of the light-curves as the time it takes for the light-curve to rise and decline 1 mag relative to its peak (as in Villar et al. 2017). This duration measurement is possible for 31 of our spline-fitted SN light-curves in Fig. A.3.

A comprehensive exploration of the DLPS for different astronomical transients was done by Villar et al. (2017), who used the MOSFiT (Modular Open Source Fitter for Transients) code (Guillochon et al. 2018) to explore the locations in the DLPS occupied by transients driven by different mechanisms (such as CSI) occurring under wide but realistic parameter ranges. Using the analytical models based on Chatzopoulos et al.

\footnotetext{
3 Spearman correlation tests (unweighted) for SN peak absolute magnitudes, rise times, and decline rates versus $M_{\mathrm{g}}$ of the host galaxies ( $\mathrm{cf}$ Sect. 4.4) give significance levels of $\sigma \lesssim 1$, i.e., no correlations can be seen.
}

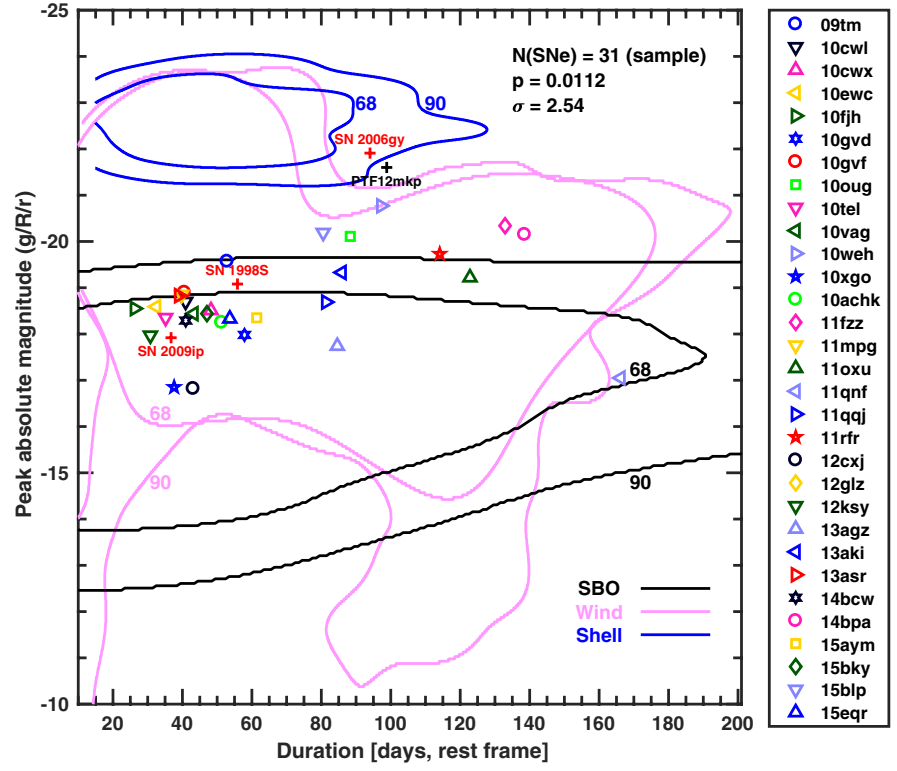

Fig. 13. Percentile contours for the DLPS of CSI-driven $\mathrm{SNe}$ as explored by Villar et al. (2017) using the MOSFiT code (Guillochon et al. 2018). SN-like events are shown in pink (wind-like CSM) and blue (shell-like CSM) whereas shock-breakout (SBO) transients are shown in black. For each contour colour, the inner contour represents the 68th percentile and the outer contour the 90th percentile. From our sample, $31 \mathrm{SNe}$ are plotted, along with the SLSN IIn PTF12mkp. For comparison, the much-studied SNe 1998S (Liu et al. 2000), 2006gy (Smith et al. 2007), and 2009ip (2012B event, Graham et al. 2014) are included as well. For the $\mathrm{N}(\mathrm{SNe})=31$ sample $\mathrm{SNe}$, the Spearman correlation coefficient $p$ and the corresponding significance $\sigma$ are shown.

(2012) for SN-like CSI transients (with both wind-like and shelllike CSM profiles) and the model by Ofek et al. (2014b) for SBO interacting transients, Villar et al. (2017) calculated 68th and 90th percentile contours for the DLPS populated by CSI-driven transients. The CSM mass in all models was picked from the range $0.1-10 M_{\odot}$, sampled log-uniformly. In Fig. 13 we compare our sample SNe to the DLPS percentile contours by Villar et al. (2017). The slight overlap of the contours in some places for the SN-like transients is due to the contours being generated based on only $1000 \mathrm{MC}$ realisations (Ashley Villar, personal communication). We also plot SLSN IIn PTF12mkp (Sect. 3). We also did spline measurements (as in Sect. 4.1) for photometry of SNe 1998S (Liu et al. 2000), 2006gy (Smith et al. 2007), and 2009ip (2012B event, Graham et al. 2014) to include them in Fig. 13 for comparison. A unweighted Spearman test for the 31 sample SNe IIn plotted in Fig. 13 gives $p=0.0112$, corresponding to a significance of $\sim 2.5 \sigma$. A correlation like this (more luminous $\mathrm{SNe}$ IIn generally evolving more slowly) could be expected, based on the investigation presented in Fig. 9. It is noteworthy that in Sect. 4.4, we found a correlation (at $2.7 \sigma$ ) between peak brightness and decline rate, but no correlation $(\sim 1 \sigma)$ between peak brightness and rise time. The overall slower evolution of more luminous SNe IIn therefore seems to be dictated by the decline rates of the $\mathrm{SNe}$.

In Fig. 13 we see that 19 of the sample SNe IIn reside within both the SBO 68th percentile and the wind-like 68th percentile, suggesting that either model could explain these $\mathrm{SNe}$. At $M \lesssim-20 \mathrm{mag}$, we see $5 \mathrm{SNe}$ outside the SBO 90th percentile but still inside the wind-like 68th percentile, making it less likely that they are SBO-driven SNe IIn. Two of the SNe plotted (comparison events PTF12mkp and SN 2006gy) are found in a region 
of the DLPS where both wind-like and shell-like CSM could offer a satisfactory explanation of their light curves.

The DLPS plot (Fig. 13) shows a noticeable clustering of events with durations between $20 \mathrm{~d}$ and $60 \mathrm{~d}$ and peak magnitudes between $\sim-18$ and $\sim-19$, whereas the more luminous $(M<-20 \mathrm{mag})$ and long-lasting events are rarer. Observationally, more long-lasting and luminous events should be easier to find and monitor, also at higher redshifts. This might indicate that the less luminous and less long-lasting SNe IIn are intrinsically more common. This can be interpreted such that progenitors with a relatively lower mass-loss rate towards the end of their existence dominate the zoo of SN IIn progenitors. A rapidly declining SN IIn likely has a less dense CSM, whereas a slowly declining SN IIn should have an extended dense CSM. The differences in extent and density of the CSM suggest a difference in the mass-loss histories of SN IIn progenitors, with the progenitors of more long-lasting and luminous SNe IIn having enhanced mass loss for decades or longer (cf. Moriya et al. 2014) before the SN explosions, while the fainter and briefer SNe IIn possibly only have stronger mass loss during the years before the SN.

\section{Discussion}

In this section, we discuss the contents of our sample. We examine what could be contaminating our sample in terms of unrelated astronomical transients, and we evaluate sources of errors. Further, we discuss some of our individual SNe and compare them to $\mathrm{SNe}$ in the literature with respect to SN impostors, lightcurve bumps, and flat light-curve maxima.

\subsection{Possible contaminants in the sample}

Transients showing SN IIn spectral signatures are usually understood to involve a supergiant star having an outburst (destructive or not) inside a substantial CSM. There are other mechanisms that can mimic the SN IIn signature and which could possibly contaminate our sample. Here, we discuss two such mechanisms.

Under some circumstances, an active galactic nucleus (AGN) can appear like a SN IIn both spectroscopically and photometrically. For example, an early label put on SNe 1987F and 1988I (later classified as SNe IIn) was "Seyfert 1-like" (Filippenko 1989). The erratic photometric behaviour of an AGN (caused e.g. by changes in accretion rate of its supermassive black hole) can lead to variability episodes (see e.g. Graham et al. 2017) with duration and absolute magnitude comparable to some SNe IIn. Most of the SNe in our sample are located off-centre in their host galaxies. Vetting The Million Quasars catalogue (version 6.3, 2019 June) compiled by Flesch (2015) shows that no known AGN is found in the catalogue within $5^{\prime \prime}$ of our SN positions. This suggests that the transients in our sample are likely not AGN in outburst mimicking SNe IIn.

Some thermonuclear SNe have been observed showing spectra with SN IIn-like Balmer lines superimposed on ordinary SN Ia spectra (e.g. Hamuy et al. 2003). These SNe Ia-CSM have been proposed to happen when white dwarfs explode inside the CSM of its non-degenerate binary companion (see Silverman et al. 2013, and references therein). In simulations by Leloudas et al. (2015) it was shown that SNe Ia-CSM can be mistaken for genuine SNe IIn under some circumstances. Leloudas et al. (2015) shows that a SN Ia (of typical peak magnitude $M \approx-19$ ) can be hidden under an apparent SN IIn spectrum if the CSI is strong and the peak magnitude of the SN IIn is $M \approx-20.9$.
In this interval, our sample contains PTF12frn (Table 6). We caution that this SN might have an overestimated host extinction (Sect. 3). PTF12frn was a part of the feeder sample for the Silverman et al. (2013) study, but its spectra made them conclude that it was not a SN Ia-CSM. It therefore seems likely that none of our sample SNe are SNe Ia-CSM.

\subsection{Sources of errors}

Some of the assumptions made when measuring the light-curve properties could affect our analysis and conclusions. Sources of error important for absolute magnitudes and colours are the extinction corrections, distances, and the bolometric correction for the $\mathrm{SNe}$. The values we obtain for $\mathrm{SN}$ decline rates and rise times are not affected by these.

No comprehensive study of bolometric corrections (BC) for $\mathrm{SNe}$ IIn has yet been done. The Balmer emission lines in their spectra as well as the infrared contributions from dust (Fox et al. 2013) makes their BC hard to estimate. For blackbody temperatures between 7500 and $11000 \mathrm{~K}$, the bolometric correction of our Mould $R$-band filter is $-0.6<\mathrm{BC}<-0.06$ mag (Ofek et al. 2014 b, footnote 22). These BC values for SNe IIn are adopted by Moriya \& Maeda (2014). For practical reasons (and following Ofek et al. 2014a; Nyholm et al. 2017), we have been using $\mathrm{BC}=0 \mathrm{mag}$ in our analysis with the $R / r$ and $g$ bands, and this likely introduces an error.

Several samples of CC SNe (e.g. Valenti et al. 2016; Taddia et al. 2015a, 2019) show host colour excess in the range $0 \lesssim E(B-V) \lesssim 0.4 \mathrm{mag}$. In the literature compilation for SN Ia host galaxies by Cikota et al. (2016), the absorptionto-reddening ratios are in the range $1 \lesssim R_{V} \lesssim 3.5$. Owing to the low sensitivity of our spectra to the Na ID absorption diagnostic (Sect. 3) it appears likely that we could underestimate the host extinction for some of the SNe in our sample, and the distribution of peak absolute magnitudes is systematically brighter than reported. As indicated by the small spread (a few tenths of a magnitude around light-curve peak epoch) in the colour evolution displayed in Fig. 10, in practice it seems unlikely that we have large extinction correction errors.

A $5 \mathrm{~km} \mathrm{~s}^{-1} \mathrm{Mpc}^{-1}$ uncertainty in $H_{0}$ corresponds to an uncertainty in the distance moduli of $0.15 \mathrm{mag}$. This is a systematic error, applying equally to all $\mathrm{SNe}$ in the sample, and thus introduces a random uncertainty in the derived luminosity function.

Concerning the selection of the sample, the $+40 \mathrm{~d}$ (after discovery) lower limit for having detections (Sect. 3) affects $\mathrm{SNe}$ with fast declines and means that the sample is not inclusive of such events. During the course of this work, it became clear that the post-peak P60 detections initially used to make PTF09bcl a member of the sample were actually upper limits. Owing to the good coverage of the PTF09bcl rise and peak, we did keep PTF09bcl in the sample despite that.

\subsection{Possible SN impostor PTF11qnf}

The question of SN impostors (e.g. Van Dyk et al. 2000; Van Dyk \& Matheson 2012) is important to address. Such transients are generally less luminous than SNe IIn, but can still display the multi-component Balmer emission lines characteristic of SNe IIn, thus mimicking some of the SN IIn behaviour and earning them the label "impostors". No CC is assumed to happen in an SN impostor. The compilation by Smith et al. (2011) shows that $\mathrm{SN}$ impostors typically have peak magnitudes in the 


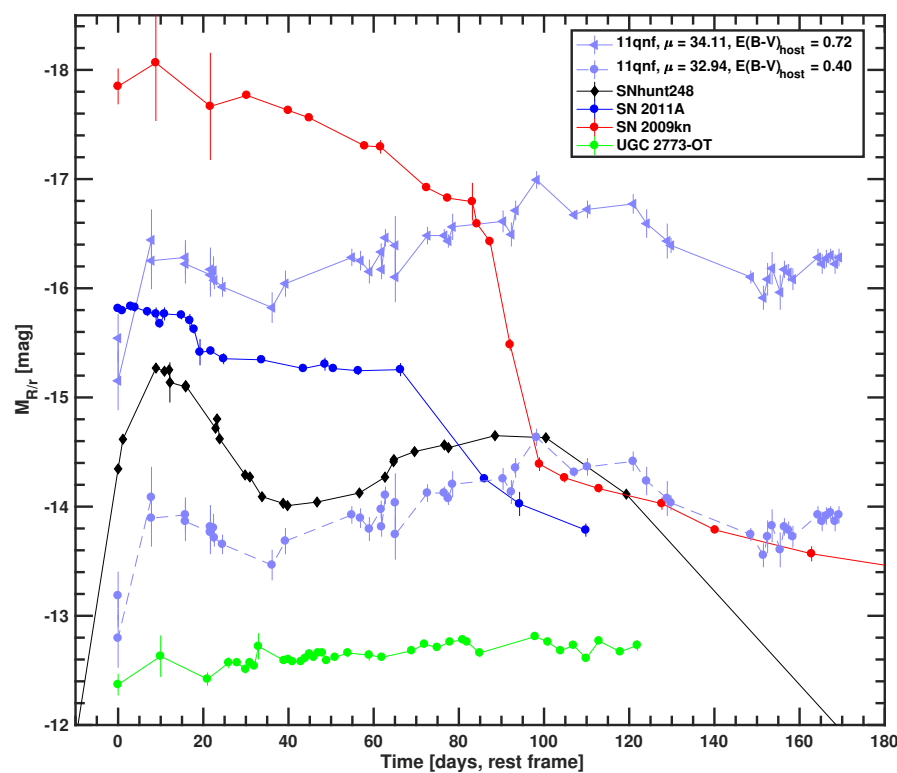

Fig. 14. Comparison between $\mathrm{SN}$ impostor candidate PTF11qnf and events from the literature $(R / r$-band absolute magnitudes). For PTF11qnf, we show the light-curve assuming two different combinations of distance modulus and host extinction: $\mu=34.11$ mag given in Table 5 and the $E(B-V)_{\text {host }}$ from Sect. 3, as well as assuming the Tully-Fisher based $\mu=32.94$ mag (Lagattuta et al. 2013) and the $E(B-V)_{\text {host }}$ estimated in Sect. 5.3. Using the latter combination of $\mu$ and $E(B-V)_{\text {host }}$ a similarity between PTF11qnf and SNhunt 248 can be discerned. SNhunt248 (Kankare et al. 2015), SN 2011A (de Jaeger et al. 2015), SN 2009kn (Kankare et al. 2012), and U2773-OT (2009 outburst; Smith et al. 2010) are shown with distance moduli and extinction values are taken from the cited papers. Epoch $0 \mathrm{~d}$ is set at the first detection in the light-curve (for PTF11qnf, SN 2009kn, and SN 2011A) or the observed start of the outburst episodes (for SNhunt248 and U2773-OT). Lines are drawn to guide the eye.

range $-16<M<-10$ mag and usually have erratic lightcurve shapes. Outbursts of LBV stars is one common mechanism invoked to explain SN impostors (Smith et al. 2011). The faint end of the peak absolute magnitude distribution for our sample (Fig. 3) lies at $M \approx-17 \mathrm{mag}$, and in this respect the majority of our sample SNe would not be regarded as SN impostors. The case of PTF11qnf, with a plateau-like and erratic light-curve peaking at $M \approx-17 \mathrm{mag}$, calls for special attention as a possible SN impostor candidate. We attempt to evaluate the nature of PTF11qnf by comparing it to other transients showing similar behaviour (Fig. 14).

An interesting comparison can be made to the transient SNHunt248, which showed a fast rise to a double-peaked maximum lasting $\gtrsim 100 \mathrm{~d}$ at $M \approx-14 \mathrm{mag}$ (Mauerhan et al. 2015; Kankare et al. 2015), interpreted by those authors as the nondestructive outburst of a hypergiant star. The deepest upper limits in the photometry from the P48 suggest that the absolute magnitude of PTF11qnf was $M_{R} \gtrsim-15$ between -960 to $-10 \mathrm{~d}$ and between +260 to $+1950 \mathrm{~d}$, relative to the first detection (JD 2455 866.9). This is not a deep limit, and still allows for pre-discovery variability such as that observed in SNHunt248 (Kankare et al. 2015), but demonstrates that the $\sim 180$ d plateau episode we observe in PTF11qnf was preceded and followed by fainter periods. SN 2011A (de Jaeger et al. 2015) had a SN IIn spectrum, a maximum observed luminosity in the range between those of SNe and SN impostors, and a light-curve having two plateaus. de Jaeger et al. (2015) favoured a SN impostor scenario for this transient. The 2009 outburst of the transient
U2773-OT (Smith et al. 2010) exhibited a light-curve plateau of $M_{R} \approx-12 \mathrm{mag}$ lasting $\sim 120 \mathrm{~d}$, as well as $\mathrm{H} \alpha$ emission with a narrow component. Smith et al. (2010) concluded that U2773-OT was a LBV in outburst, and later studies of its $\gtrsim 15 \mathrm{yr}$ light-curve led Smith et al. (2016) to compare U2773-OT to the LBV star $\eta$ Car. While there is a spread of $\sim 4$ mag in maximum brightness, the duration of the light-curves of SNHunt248, SN 2011A, and U2773-OT are similar. For comparison, Fig. 14 also shows SN 2009kn (Kankare et al. 2012), a SN IIn which had a plateau-like behaviour on a time scale similar to that of PTF11qnf, but was initially more luminous and most likely was a CC SN (Kankare et al. 2012).

The single spectrum we have of PTF11qnf (Fig. A.1) was taken during its initial hump and shows a flat continuum with conspicuous $\mathrm{H} \alpha$ and $\mathrm{H} \beta$ emission. A Gaussian fit to its $\mathrm{H} \alpha$ narrow component gives a full width at half-maximum of $\sim 400 \mathrm{~km} \mathrm{~s}^{-1}$. This spectrum is comparable to some of the LBV outburst spectra compiled by Smith et al. (2011), as well as to the early spectra of SN 2011A (de Jaeger et al. 2015). The Fe II lines reported by Kankare et al. (2015) for SNhunt248 were not seen in PTF11qnf, but otherwise their spectra are comparable.

The redshift of PTF11qnf is the lowest in our sample and its host (UGC 3344) is located in a group of galaxies (LDC 410; Crook et al. 2007). This makes the distance estimate based on redshift particularly uncertain. Using the Tully-Fisher method, Lagattuta et al. (2013) obtained $\mu=32.94$ mag for the host of PTF11qnf. In Fig. 10, $E(B-V)_{\text {host }}=0.72 \mathrm{mag}$ was assumed based on $\mathrm{Na} I \mathrm{D}$ absorption, but this gives a conspicuous offset in $g-i$ towards the blue, compared to all other entries in this figure. The slope of the spectrum of PTF11qnf as well as the redder colours reported for SNHunt248 by Kankare et al. (2015) suggests that we might have overestimated the colour excess from the PTF11qnf host. Instead assuming $E(B-V)_{\text {host }}=0.4$ mag would put PTF11qnf within the $g-i$ range of the other entries in Fig. 10. Using the Tully-Fisher $\mu$ and $E(B-V)_{\text {host }}=0.4 \mathrm{mag}$, Fig. 14 shows that PTF11qnf might be more similar in luminosity to SNHunt248, having a late-time evolution similar to the second bump of SNHunt248.

The spectroscopic and photometric similarities of PTF11qnf to a number of transients interpreted as nondestructive outbursts of massive stars points towards this transient not being a SN, but rather an impostor. For this reason we removed PTF11qnf from the analysis in Sects. 4.3 and 4.4.

\subsection{Bumpy SN IIn light-curves}

The light-curves of some SNe IIn have breaks in their postpeak decline, when the SNe temporarily re-brightens before the decline in brightness resumes. Such episodes appear as a bump in the light-curve and have been seen in, for example, SNe IIn 2006jd (Stritzinger et al. 2012), 2009ip (Margutti et al. 2014; Graham et al. 2014; Martin et al. 2015), and iPTF13z (Nyholm et al. 2017). The bumps have been proposed (e.g. Graham et al. 2014; Nyholm et al. 2017) to happen when SN ejecta encounter regions of increased density in the CSM.

Figure A.2 indicates that some $\mathrm{SNe}$ in our sample have lightcurve bumps. Photometry of the PTF10tel bump was presented by Ofek et al. (2013) while some of the other bumps have not been discussed before. The possible bump of PTF10weh ( $\sim 0.2$ mag break in the $r$-band decline, $\sim 116 \mathrm{~d}$ after peak) seems to occur also in the Bgi bands, while the start of the possible bump of iPTF13aki ( $\sim 0.4$ mag rise in the $r$-band, $120 \mathrm{~d}$ after peak) is possibly also captured in the $i$-band. Whereas the PTF10weh bump could be a true, (pseudo)bolometric bump, the 


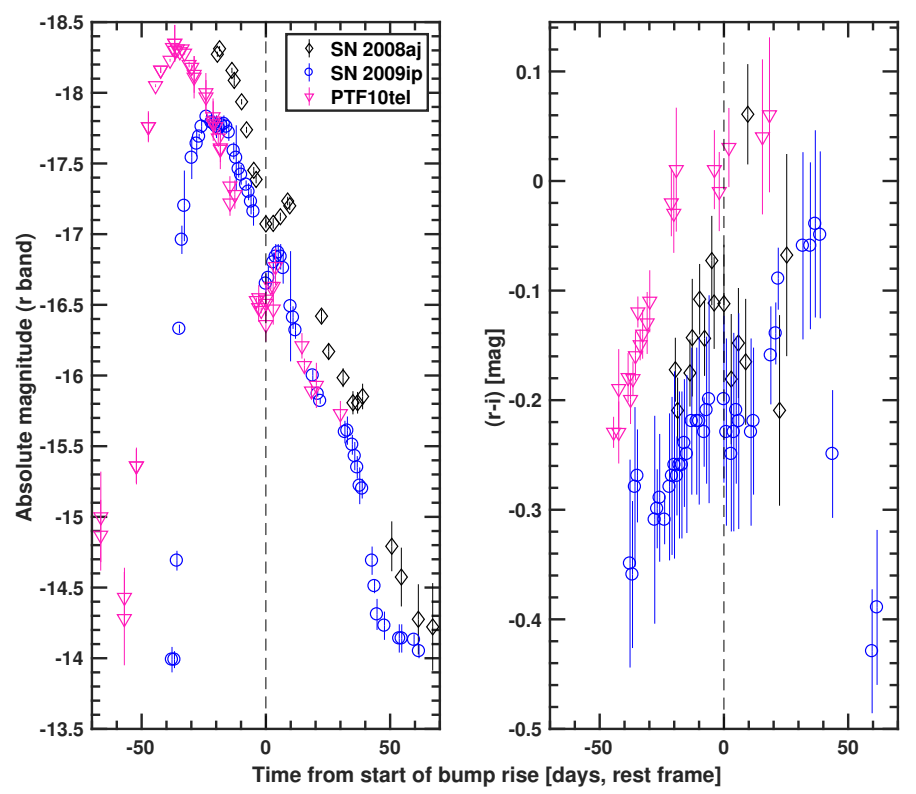

Fig. 15. Comparison of the $r$-band absolute magnitudes and $r-i$ colours for the bumps seen in Type IIn SNe 2008aj, 2009ip (2012B event), and PTF10tel. Photometry and extinction corrections are taken from Hicken et al. (2017), Graham et al. (2014), and this paper, respectively. For clarity, colour data points with uncertainties $>0.1$ mag are not shown. The vertical, dashed black line indicates the estimated start epoch of the light curve bumps.

nature of the possible iPTF13aki bump is harder to evaluate. The difficulty in judging the nature of light-curve bumps is illustrated by the light-curve of PTF11fzz.

For PTF11fzz, the quite conspicuous bump ( $\sim 0.5 \mathrm{mag}$ amplitude) seen around $197 \mathrm{~d}$ after peak is likely a consequence of the $\mathrm{H} \alpha$ line centre being shifted to $7097 \AA$ at the redshift of PTF11fzz $(z=0.081)$. This puts the $\mathrm{H} \alpha$ emission inside the Mould $R$ filter, but outside the SDSS $r$ filter. We lack spectra of PTF11fzz around this epoch, but a comparison to PTF12glz (at the similar $z=0.079)$ is instructive. A large offset $\left(R_{\text {Mould }}-\right.$ $r_{\text {SDSS }} \approx-0.8 \mathrm{mag}$ ) can be seen (Fig. A.2) in our $R / r$ photometry of PTF12glz around $220 \mathrm{~d}$ after its estimated peak epoch. We have a spectrum of PTF12glz taken about $235 \mathrm{~d}$ after its estimated peak epoch (the JD 2456422 Keck-I/LRIS spectrum from Soumagnac et al. 2019), which is comparable to the epoch where we see the apparent bump in PTF11fzz. Synthetic photometry on this PTF12glz spectrum using synphot by Ofek (2014) gives a $R_{\text {Mould }}-r_{\text {SDSS }} \approx-0.6 \mathrm{mag}$ difference. This is comparable to the offset we see in the observed photometry. The large offsets $\left(R_{\text {Mould }}-r_{\text {SDSS }}\right)$ seen in two different SNe IIn both at $z \approx 0.08$, and confirmed via synthetic photometry for one of them (PTF12glz), makes it likely that the "bump" we see in PTF11fzz is a photometric filter effect.

The precursor activity as well as the bump of both PTF10tel ( $\sim 40$ d past peak) and SN 2009ip ( 40 d past peak of the 2012B event) has been noted before (Ofek et al. 2013; Graham et al. 2014; Smith et al. 2014). Adding to this, in the data release by Hicken et al. (2017) we see that the Type IIn SN 2008aj has a post-peak decline and a bump comparable to bumps seen in PTF10tel and SN 2009ip. Pastorello et al. (2018) point out the similarity between SN 2009ip and a number of events with similar rise and decline rates, as well as comparable peak brightness. Here, we add SN 2008aj to that comparison and emphasise the presence of light-curve bumps.
For all three $\mathrm{SNe}$, we plot the $r$-band light-curves and the $r-i$ colour in Fig. 15. A common property of the three bumps is that their rises last $\sim 10 \mathrm{~d}$ and the height of the rise is $\sim 0.3 \mathrm{mag}$. We also note that the $r$-band decline rates pre-peak and postpeak are comparable. In $r-i$ colour, SNe 2008aj and 2009ip display a somewhat more similar behaviour pre-peak compared to PTF10tel, but all three SNe show a general change to redder colour before the bump start. This change slows down around the start of the bump, to resume afterwards. For comparison, the $r-i$ colour of the most long-lasting bump of iPTF13z (called $B_{3}$ by Nyholm et al. 2017) showed a reddening before the bump, but turned bluer as the bump ended. As in iPTF13z, the bumps shown in Fig. 15 all appear in multiple optical bands, suggesting that they are driven by the continuum of the SNe rather than by (for example) $\mathrm{H} \alpha$ evolution (as in the bump of SN 2006jd; see Stritzinger et al. 2012). For SN 2009ip, an LBV star has been proposed as the progenitor (e.g. Mauerhan et al. 2013b; Smith 2017b). The remarkable similarity between the evolution of SN 2008aj, SN 2009ip and PTF10tel around main peak as shown in Fig. 15 might indicate similar progenitors (e.g. LBV stars) for all three events.

\subsection{The frequency of SN IIn light-curve bumps}

Bumps in SN IIn light-curves are apparently rare. Conspicuous light-curve bumps have been observed in $\lesssim 10$ SNe IIn, that is in $\lesssim 2 \%$ of the $\approx 550$ public SNe IIn listed in the Open Supernova Catalog (OSC; Guillochon et al. 2017) as of 2019 September. We used our observations of SNe IIn from PTF/iPTF and MC simulations of detection probability to constrain the frequency of SN IIn bumps.

The bumps are intrinsically dissimilar, in terms of amplitudes, durations, shapes, and time of occurrence in the light curve. For simplicity, we consider bump $B_{1}$ of iPTF13z (Nyholm et al. 2017) to be our model bump. Fitting a second-degree polynomial to the $R$-band photometry of iPTF13z between 125 and 162 rest-frame days after discovery gives $m(t)=0.0016\left(t-t_{0}\right)^{2}-$ $0.51+\Delta m$ mag as a simple model of bump $B_{1}$. The model is valid within 0.51 mag of bump peak at $t_{0}$. We draw a magnitude offset $\Delta m$ uniformly between 1 and 3 mag fainter than the SN peak and a time offset uniformly between 50 and $200 \mathrm{~d}$ after SN peak for the bump to start. This random offset relative to the peak apparent magnitude of a SN in our sample introduces our model bump in that $\mathrm{SN}$ in a way resembling how the bump appeared in iPTF13z. We use the SN peak apparent magnitudes, but do not take any other light-curve property of the sample SNe into account.

To represent how SN IIn photometry is obtained, for each $\mathrm{SN}$ in our sample we consider all epochs when the $\mathrm{SN}$ was observed (after discovery) with the P48 and P60 in the photometric bands listed in Table 6 . Such sets of epochs are representative for how PTF/iPTF operated, also capturing interruptions caused by for example cloudy nights or solar conjunction gaps. When the model bump has been introduced at a random place relative to the peak of a sample SN, the bump (stretched in time to the observer's frame) is sampled using the photometric epochs for that SN. The bump is considered to be detected if the following criteria are fulfilled: $\geq 3$ epochs fall on the bump, with a time of $\geq 10 \mathrm{~d}$ separating the first and the last such epoch, and the faintest of the epochs giving $m \leq 20.5 \mathrm{mag}$.

Introducing 100 such model bumps randomly (as described above) in each of the $39 \mathrm{SNe}$ of our sample with measured peak epoch (Table 6) gives an acceptance fraction of $\sim 8.5 \%$, that is the bump being detected in $\sim 8.5 \%$ of the cases. From 
our observations, we know that no bump like $B_{1}$ of iPTF13z was seen in these $39 \mathrm{SNe}$ in the sample. A smaller bump in PTF10tel was indeed seen, but in this MC experiment we consider only our model bump from iPTF13z. Knowing that 1 bump ( $B_{1}$ itself) was detected among the 69 remaining SNe IIn from PTF/iPTF ( $\sim 1.5 \%$; see Table 4$)$ allows us to make a quantitative estimate of the fraction of SNe IIn displaying light-curve bumps.

We model the observation as independent observations of $N_{a}=39 \mathrm{SNe}$ IIn, calling this sample " $a$ ", with a probability $p_{a} \cdot \alpha$ for a bump to be detected in one SN IIn. Here, $\alpha$ is the probability of SNe to feature a bump, and $p_{a}=8.5 \%$ is the probability for this bump to be detected. In addition to this sample, we have sample " $b$ ", with $N_{b}=69$ SNe IIn with a bump probability $p_{\mathrm{b}} \cdot \alpha$. For this sample, we do not know the bump detection probability, and conservatively allow it to take any value between 0 and $1^{4}$. We model the observations as two independent samplings of $\mathrm{SNe}$ with a different probability to detect a bump, disregarding possible correlations due to selecting the samples. The total likelihood for the observation of $n_{a}=0$ and $n_{b}=1$ in the two samples may be written as the product of two binomial distributions, with $\alpha$ and $p_{\mathrm{b}}$ as parameters:

$L\left(\alpha, p_{b}\right)=\operatorname{Bin}\left(n_{a} \mid N_{a}, p_{a} \cdot \alpha\right) \cdot \operatorname{Bin}\left(n_{b} \mid N_{b}, p_{b} \cdot \alpha\right)$.

The maximum likelihood estimate of $\alpha$ is 0.014 . Using the profile likelihood ratio and the assumption of an asymptotically distributed test statistic (Wilks' theorem), the $1 \sigma$ confidence interval for $\alpha$ is $[0.0043,0.16]$. A closer examination of the likelihood surface showed that the upper constraint on $\alpha$ is dominated by the term due to sample " $a$ ", while the lower edge is set by the observation of one bump in sample " $b$ ". Improved knowledge of $p_{b}$ may improve the lower constraint. With the current unconstrained probability, the profiled likelihood raises $p_{b}$ to 1 for low $\alpha$.

From our 39 sample SNe IIn considered here and our MC experiment, we therefore estimate that bumps like the $B_{1}$ bump of iPTF13z happen in approximately $1.4_{-1.0}^{+14.6 \%}$ of all SNe IIn. This interval, for our specific case of $B_{1}$, is also consistent with our rough initial estimate based on SN IIn bumps in the literature and the number of SNe IIn from the OSC.

Given the assumption that SN IIn light-curve bumps are caused by SN ejecta encountering denser regions of CSM, the rarity of such bumps is noteworthy. Modelling (e.g. Kochanek 2009) and observations (Sana et al. 2012; Dunstall et al. 2015, but see also Kochanek 2018) suggest that at least $50 \%$ of massive stars reside in binary or multiple systems. In such binaries, the mass lost by the constituent stars can be shaped into spiral patterns (Tuthill et al. 2008) which could cause light-curve bumps if one of the stars explodes as a CSI-dominated SN (as invoked by Schwarz \& Pringle 1996, for SN 1979C). The LBV stars, often proposed as SN IIn progenitors, are known to have structured CSM (e.g. Weis 2011) which could also lead to bumpy light-curves if swept up by SN ejecta. Selection effects due to geometry and viewing angle might be at work, preventing lightcurve bumps from occurring or being seen in the majority of SNe IIn. Distinctly non-spherical geometries have been demonstrated using spectropolarimetry for SNe IIn 1997eg (Hoffman et al. 2008), 1998S (Leonard et al. 2000), 2009ip (Reilly et al. 2017), and 2010jl (Patat et al. 2011). The relative rarity of light-curve bumps might be a separate indicator of non-spherical geometries in SNe IIn, or that CSM density changes big enough to cause conspicuous bumps somehow are hard to make.

\footnotetext{
4 Constraining $p_{b}<p_{a}$, which could be a reasonable assumption as this sample is sampled less, will move the lower edge of our confidence level up slightly.
}

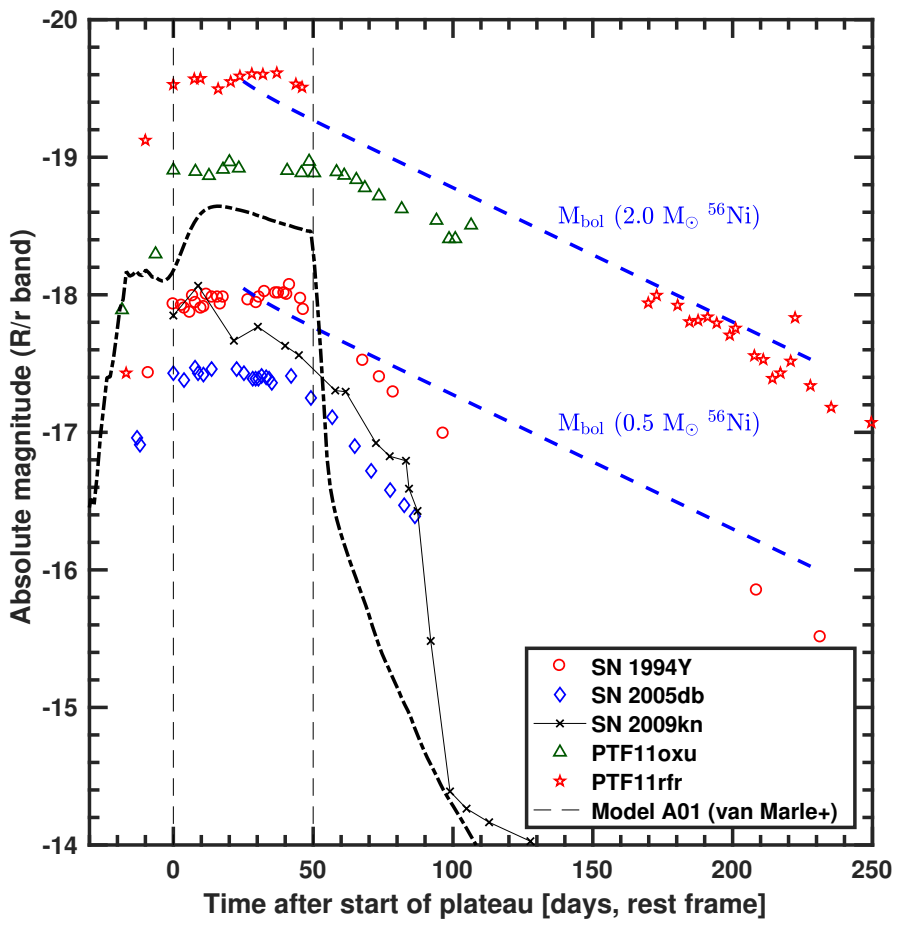

Fig. 16. Compilation of SNe IIn with rapid rises, plateau-like maxima, and linear declines. Shown here are the sample SNe PTF11oxu and PTF11rfr (photometry binned in 3 day bins for clarity) along with SNe 1994Y (Ho et al. 2001) and 2005db (Kiewe et al. 2012). For comparison, the Type IIn-P SN 2009kn (Kankare et al. 2012) is shown. Dashed vertical lines highlight $50 \mathrm{~d}$ plateau duration. The bolometric decay rates (Nadyozhin 1994) for 0.5 and $2.0 M_{\odot}$ of synthesised ${ }^{56} \mathrm{Ni}$ are shown as dashed blue lines (assuming a rise time to plateau start of $20 \mathrm{~d}$ ) for comparison. They are not fits to the photometry. Model A01 (bolometric luminosity) by van Marle et al. (2010) is also shown.

A simpler explanation might be the bias caused by the short ( $\sim 100$ d) time window after the SN explosion during which we can discover the bumps. For typical CSM and SN ejecta velocities, a SN progenitor outburst must occur shortly before the SN in order to produce a suitably located density change causing a bump we can actually observe.

\subsection{SNe IIn with plateaus and slow declines}

Plateaus have been seen in the light-curves of some SNe IIn, earning them the designation Type IIn-P (where " $P$ " stands for plateau). Examples are SNe 1994W (Sollerman et al. 1998), 2009kn (Kankare et al. 2012) and 2011ht (Fraser et al. 2013; Mauerhan et al. 2013a), which all exhibited plateau-like maxima lasting $\sim 100$ days followed by a rapid decline $\left(\sim 0.25 \mathrm{mag} \mathrm{d}^{-1}\right)$ from the plateau. Different models have been proposed to explain the Type IIn-P SNe, for example colliding CSM shells (see Dessart et al. 2016, and references therein).

In our sample, we see a version of the SNe IIn-P with a behaviour different from the examples above. Instead of dropping quickly in brightness after the plateau, these SNe instead showed a slower, linear decline after their plateaus. Among our sample SNe, we see that PTF11oxu and PTF11rfr have a rather fast rise to the plateau (rise time to plateau start $\lesssim 25 \mathrm{~d}$ ) and a plateau lasting $\gtrsim 50 \mathrm{~d}$, followed by a linear decline. The plateaus occur at $M_{r} \approx-19 \mathrm{mag}$ and $M_{r} \approx-19.6 \mathrm{mag}$ for PTF11 oxu and PTF11rfr, respectively. The spectral sequence of PTF11rfr opens 
a possibility that the narrow component of its $\mathrm{H} \alpha$ line used to classify it as a SN Type IIn might come from the host galaxy. The plateau magnitude of $M_{r} \approx-19.6$ mag for PTF11rfr is significantly more luminous than the average SN Type IIP peak magnitude of $M_{B} \approx-16.75 \pm 0.98$ mag (Richardson et al. 2014) making CSI a plausible scenario for PTF11rfr.

Vetting the literature, we find that photometry of SNe 1994Y (Ho et al. 2001) and 2005db (Kiewe et al. 2012) show comparable, but intrinsically fainter, $R / r$-band light-curves. These light-curves are plotted in Fig. 16. The $I$-band light-curve of Type IIn SN OGLE 2013-SN-016 (Wyrzykowski et al. 2013; Nicholl et al. 2013, raw photometry by the Optical Gravitational Lensing Experiment, OGLE) appears to have had a similar plateau duration and decline phase. Figure 16 shows that the spread in plateau absolute magnitude is $\gtrsim 2$ mag and that the decline rates differ after the end of the plateau. The models by van Marle et al. (2010) of SNe IIn, where the SN ejecta collide with a CSM shell, can produce light-curves with plateaus of approximately the right duration and luminosity; see van Marle et al. (2010, their Figs. 11 and 12). As an example, in Fig. 16 we plot model A01 by van Marle et al. (2010), where SN ejecta hit a $1 M_{\odot}$ CSM shell. The steep rise and the duration of the model A01 plateau, as well as the plateau luminosity of this model, is comparable to the SNe shown. However, model A01 (and the other van Marle et al. 2010 models) gives a too steep decline after the plateau compared to the SNe of our proposed sub-type. The slow decline of the SNe IIn here could be due to an extended CSM outside the shell. While Type IIn-P (like SN 1994W, with fast decline) have been studied by several authors, the SNe IIn with plateaus and slow declines warrants further investigation to determine if they constitute a distinct sub-type of SNe IIn.

\section{Conclusions}

We have studied a sample of $42 \mathrm{SNe}$ IIn from the untargeted PTF/iPTF survey. Our main conclusions are summarised below.

The luminosity function of SNe IIn, after compensating for Malmquist bias and K-corrections, is $M_{\text {peak }}=-18.72 \pm 1.32 \mathrm{mag}$ (if applying the $M>-21$ mag cut to exclude ostensibly "superluminous" $\mathrm{SNe}$ ) or $M_{\text {peak }}=-19.18 \pm 1.32 \mathrm{mag}$ (if that cut is not applied, and all SNe with Type IIn spectral signatures are included). The typical rise times to peak for SNe IIn are $20 \pm 6 \mathrm{~d}$ and $50 \pm 11 \mathrm{~d}$, dividing the SNe IIn into fast and slow risers. The typical decline rates of SNe IIn for the initial $50 \mathrm{~d}$ after light-curve peak can be described with the Gaussian distribution $0.027 \pm 0.016 \mathrm{mag} \mathrm{d}^{-1}$. A bimodal distribution possibly exists, with slower and faster declines (with slopes $0.013 \pm 0.006 \mathrm{mag} \mathrm{d}^{-1}$ and $0.040 \pm 0.010 \mathrm{mag} \mathrm{d}^{-1}$ ).

We find no strong correlation between the peak luminosity of SNe IIn and their rise times. A correlation between the peak luminosity and the duration within $1 \mathrm{mag}$ of peak was found. Correlations between rise time and decline rate, and peak absolute magnitude and decline rate, can also be discerned.

The colour evolution of SNe IIn is rather uniform. The colour indices at peak of the sample SNe IIn are $g-r=0.06 \pm 0.21 \mathrm{mag}$ and $g-i=0.13 \pm 0.46 \mathrm{mag}$. K-corrections for SNe IIn at lightcurve peak are found to be within 0.2 mag for the observer's frame $r$-band, for SNe IIn at $z<0.25$.

The $\mathrm{SNe}$ in the sample were hosted by galaxies of absolute magnitude $-22 \lesssim M_{\mathrm{g}} \lesssim-13 \mathrm{mag}$, that is with global metallicities ranging from supersolar to subsolar. No correlations between host global metallicity and SN IIn rise times, decline rates, or peak absolute magnitude were found.
We suggest a possible new variety of "SNe IIn-P", with a light-curve showing a quick rise to a $\gtrsim 50 \mathrm{~d}$ plateau, followed by a slow, linear decline. Example events include PTF11oxu and PTF11rfr, as well as SNe 1994Y and 2005db. They are thus unlike the prototypical SNe IIn-P events such as SNe 1994W and $2009 \mathrm{kn}$, which dropped much faster from their plateaus. Lightcurve bumps like the $B_{1}$ bump of iPTF13z are rare, and happen in an estimated $1.4_{-1.0}^{+14.6} \%$ of the SNe IIn.

Acknowledgements. A.N. gratefully acknowledges a grant from Stiftelsen Gustaf och Ellen Kobbs stipendiefond, and wishes to thank Ashley Villar for providing MOSFiT contours in table format for Fig. 13, Axel Runnholm for help with Python, Ofer Yaron for help with WISeREP, Timothy E. Holy for making the distinguishable_colors function, Maayane Soumagnac and Peter Lundqvist for discussions, and opponent Philip James for questions and comments at the $\mathrm{PhD}$ thesis defence in Stockholm 2019 September 23. We gratefully acknowledge support from the Knut and Alice Wallenberg Foundation. The Oskar Klein Centre is funded by the Swedish Research Council. The intermediate Palomar Transient Factory project is a scientific collaboration among the California Institute of Technology, Los Alamos National Laboratory, the University of Wisconsin (Milwaukee), the Oskar Klein Centre, the Weizmann Institute of Science, the TANGO Program of the University System of Taiwan, and the Kavli Institute for the Physics and Mathematics of the Universe. This work was supported by the GROWTH project funded by the National Science Foundation under Grant No 1545949. A.V.F. is grateful for financial assistance from National Science Foundation (NSF) grant AST-1211916, the TABASGO Foundation, the Christopher R. Redlich Fund, and the Miller Institute for Basic Research in Science (U. C. Berkeley). E.O.O. is grateful for support by grants from the Israeli Ministry of Science, ISF, Minerva, BSF, BSF transformative program, Weizmann-UK, and the I-CORE Program of the Planning and Budgeting Committee and the Israel Science Foundation (grant No. 1829/12). G.L. was supported by research grant 19054 from VILLUM FONDEN. Part of this research was carried out at the Jet Propulsion Laboratory, California Institute of Technology, under a contract with the National Aeronautics and Space Administration (NASA). SED Machine is based upon work supported by the National Science Foundation under Grant No. 1106171. Based in part on observations obtained at the Gemini Observatory, under programs: GN-2010B-Q13 and GN-2010A-Q20 (PI: Howell), which is operated by the Association of Universities for Research in Astronomy, Inc., under a cooperative agreement with the NSF on behalf of the Gemini partnership: the NSF (United States), National Research Council (Canada), CONICYT (Chile), Ministerio de Ciencia, Tecnología e Innovación Productiva (Argentina), Ministério da Ciência, Tecnologia e Inovação (Brazil), and Korea Astronomy and Space Science Institute (Republic of Korea). Partly based on observations obtained with the Apache Point Observatory $3.5 \mathrm{~m}$ telescope, which is owned and operated by the Astrophysical Research Consortium. Some of the data presented herein were obtained at the W. M. Keck Observatory, which is operated as a scientific partnership among the California Institute of Technology, the University of California, and NASA. The Observatory was made possible by the generous financial support of the W. M. Keck Foundation. The authors wish to recognise and acknowledge the very significant cultural role and reverence that the summit of Maunakea has always had within the indigenous Hawaiian community. We are most fortunate to have the opportunity to conduct observations from this mountain. Partly based on observations at Kitt Peak National Observatory, National Optical Astronomy Observatory, which is operated by the Association of Universities for Research in Astronomy (AURA) under cooperative agreement with the NSF. The authors are honoured to be permitted to conduct astronomical research on Iolkam Du'ag (Kitt Peak), a mountain with particular significance to the Tohono O'odham. Partly based on observations made with the Nordic Optical Telescope, operated by the Nordic Optical Telescope Scientific Association at the Observatorio del Roque de los Muchachos, La Palma, Spain, of the Instituto de Astrofisica de Canarias. Partly based on observations made with the University of Hawaii's $2.2 \mathrm{~m}$ telescope, at Maunakea Observatory, Hawaii, USA. Partly based on observations made with the William Herschel Telescope operated on the island of La Palma by the Isaac Newton Group of Telescopes in the Spanish Observatorio del Roque de los Muchachos of the Instituto de Astrofísica de Canarias. Partly based on observations made with the $5 \mathrm{~m}$ Hale Telescope (P200), at Palomar Observatory, California, USA. Partly based on observations made with the Kast spectrograph on the Shane $3 \mathrm{~m}$ telescope at Lick Observatory, Mount Hamilton, California, USA. Research at Lick Observatory is partially supported by a generous gift from Google. We thank S. Bradley Cenko, Kelsey I. Clubb, Melissa L. Graham, Michael T. Kandrashoff, Patrick L. Kelly, Alekzandir Morton, Peter E. Nugent, and Jeffrey M. Silverman for help with some of the observations and reductions. This research used the Latest Supernovae web page (maintained by D. Bishop) and the Open Supernova Catalog (maintained by J. Guillochon and J. Parrent). 
This research used the SIMBAD and VizieR databases operated at CDS, Strasbourg, France, as well as the NASA/IPAC Extragalactic Database (NED) which is operated by the Jet Propulsion Laboratory, California Institute of Technology, under contract with NASA, and NASA's Astrophysics Data System. STSDAS and PYRAF are products of the Space Telescope Science Institute, which is operated by AURA for NASA. We acknowledge the whole PTF and IPTF collaborations for finding and following the $\mathrm{SNe}$ IIn studied in this sample.

\section{References}

Ackermann, M., Arcavi, I., Baldini, L., et al. 2015, ApJ, 807, 169

Anderson, J. P. 2019, A\&A, 628, A7

Anderson, J. P., \& Soto, M. 2013, A\&A, 550, A69

Anderson, J. P., James, P. A., Habergham, S. M., Galbany, L., \& Kuncarayakti, H. 2015, PASA, 32, e019

Arbour, R. 2015, Cent. Bur. Electron. Telegr., 4171, 1

Aretxaga, I., Benetti, S., Terlevich, R. J., et al. 1999, MNRAS, 309, 343

Arcavi, I., Horesh, A., Sesar, B., et al. 2010a, ATel, 3095, 1

Arcavi, I., Gal-Yam, A., Kasliwal, M. M., et al. 2010b, ApJ, 721, 777

Arcavi, I., Gal-Yam, A., Ofek, E., et al. 2013, ATel, 5407, 1

Arnouts, S., Cristiani, S., Moscardini, L., et al. 1999, MNRAS, 310, 540

Bazin, G., Palanque-Delabrouille, N., Rich, J., et al. 2009, A\&A, 499, 653

Ben-Ami, S., Badenes, C., Kulkarni, S. R., et al. 2010, ATel, 2961, 1

Benetti, S., Cappellaro, E., Danziger, I. J., et al. 1998, MNRAS, 294, 448

Bilinski, C., Smith, N., Li, W., et al. 2015, MNRAS, 450, 246

Blagorodnova, N., Neill, J. D., Walters, R., et al. 2018, PASP, 130, 035003

Blondin, S., \& Tonry, J. L. 2007, ApJ, 666, 1024

Cardelli, J. A., Clayton, G. C., \& Mathis, J. S. 1989, ApJ, 345, 245

Di Carlo, E., Massi, F., Valentini, G., et al. 2002, ApJ, 573, 144

Challis, P., Foley, R. J., \& Berlind, P. 2010, Cent. Bur. Electron. Telegr., 2243, 1

Chevalier, R. A., \& Fransson, C. 1994, ApJ, 420, 268

Cao, Y., Nugent, P. E., \& Kasliwal, M. M. 2016, PASP, 128, 114502

Cenko, S. B., Fox, D. B., Moon, D.-S., et al. 2006, PASP, 118, 1396

Chatzopoulos, E., Wheeler, J. C., \& Vinko, J. 2012, ApJ, 746, 121

Chilingarian, I. V., Melchior, A.-L., \& Zolotukhin, I. Y. 2010, MNRAS, 405 1409

Cikota, A., Deustua, S., \& Marleau, F. 2016, ApJ, 819, 152

Cowen, D. F., Franckowiak, A., \& Kowalski, M. 2010, Astropart. Phys., 33, 19

Crook, A. C., Huchra, J. P., Martimbeau, N., et al. 2007, ApJ, 655, 790

de Boor, C. 2001, A Practical Guide to Splines (New York: Springer)

Davis, T. M., Mörtsell, E., Sollerman, J., et al. 2007, ApJ, 666, 716

Drake, A. J., Djorgovski, S. G., Mahabal, A. A., et al. 2010, ATel, 2555, 1

Drake, A. J., Djorgovski, S. G., Graham, M. J., et al. 2011, Cent. Bur. Electron. Telegr., 2948, 1

Dessart, L., Hillier, D. J., Gezari, S., Basa, S., \& Matheson, T. 2009, MNRAS, 394, 21

Dessart, L., Hillier, D. J., Audit, E., Livne, E., \& Waldman, R. 2016, MNRAS, 458, 2094

de la Rosa, J., Roming, P., Pritchard, T., \& Fryer, C. 2016, ApJ, 820, 74

Dunstall, P. R., Dufton, P. L., Sana, H., et al. 2015, A\&A, 580, A93

Duszanowicz, G. 2010, Cent. Bur. Electron. Telegr., 2241, 1

Faran, T., Taubenberger, S., Kromer, M., et al. 2016, ATel, 8701, 1

Fassia, A., Meikle, W. P. S., Vacca, W. D., et al. 2000, MNRAS, 318, 1093

Fassia, A., Meikle, W. P. S., Chugai, N., et al. 2001, MNRAS, 325, 907

Filippenko, A. V. 1989, AJ, 97, 726

Filippenko, A. V. 1997, ARA\&A, 35, 309

Flesch, E. W. 2015, PASA, 32, e010

Fox, O. D., Filippenko, A. V., Skrutskie, M. F., et al. 2013, AJ, 146, 2

Fraser, M., Magee, M., Kotak, R., et al. 2013, ApJ, 779, L8

Fransson, C., Chevalier, R. A., Filippenko, A. V., et al. 2002, ApJ, 572, 350

Fransson, C., Ergon, M., Challis, P. J., et al. 2014, ApJ, 797, 118

Fremling, C., Sollerman, J., Taddia, F., et al. 2016, A\&A, 593, A68

Fukugita, M., Ichikawa, T., Gunn, J. E., et al. 1996, AJ, 111, 1748

Galbany, L., Anderson, J. P., Sánchez, S. F., et al. 2018, ApJ, 855, 107

Gal-Yam, A. 2012, Science, 337, 927

Gal-Yam, A. 2019, ARA\&A, 57, 305

Gal-Yam, A., Ben-Ami, S., Arcavi, I., et al. 2012, ATel, 4293, 1

Ganot, N., Gal-Yam, A., Ofek, E. O., et al. 2016, ApJ, 820, 57

Graham, M. L., Sand, D. J., Valenti, S., et al. 2014, ApJ, 787, 163

Graham, M. J., Djorgovski, S. G., Drake, A. J., et al. 2017, MNRAS, 470, 4112

Guillochon, J., Parrent, J., Kelley, L. Z., \& Margutti, R. 2017, ApJ, 835, 64

Guillochon, J., Nicholl, M., Villar, V. A., et al. 2018, ApJS, 236, 6

González-Gaitán, S., Tominaga, N., Molina, J., et al. 2015, MNRAS, 451, 2212

Grogin, N. A., Kocevski, D. D., Faber, S. M., et al. 2011, ApJS, 197, 35

Habergham, S. M., Anderson, J. P., James, P. A., \& Lyman, J. D. 2014, MNRAS, 441,2230

Harutyunyan, A. H., Pfahler, P., Pastorello, A., et al. 2008, A\&A, 488, 383
Hamuy, M. 2003, ApJ, 582, 905

Hamuy, M., Phillips, M. M., Suntzeff, N. B., et al. 2003, Nature, 424, 651

Hicken, M., Friedman, A. S., Blondin, S., et al. 2017, ApJS, 233, 6

Hinshaw, G., Larson, D., Komatsu, E., et al. 2013, ApJS, 208, 19

Ho, W. C. G., Van Dyk, S. D., Peng, C. Y., et al. 2001, PASP, 113, 1349

Hoffman, J. L., Leonard, D. C., Chornock, R., et al. 2008, ApJ, 688, 1186

Howell, D. A., \& Murray, D. 2012, Cent. Bur. Electron. Telegr., 3313, 2

Howell, D. A., Sullivan, M., Perrett, K., et al. 2005, ApJ, 634, 1190

Humphreys, R. M., \& Davidson, K. 1994, PASP, 106, 1025

Ilbert, O., Arnouts, S., McCracken, H. J., et al. 2006, A\&A, 457, 841

Inserra, C., Smartt, S. J., Gall, E. E. E., et al. 2018, MNRAS, 475, 1046

de Jaeger, T., Anderson, J. P., Pignata, G., et al. 2015, ApJ, 807, 63

Kankare, E., Ergon, M., Bufano, F., et al. 2012, MNRAS, 424, 855

Kankare, E., Kotak, R., Pastorello, A., et al. 2015, A\&A, 581, L4

Kelly, P. L., \& Kirshner, R. P. 2012, ApJ, 759, 107

Khazov, D., Yaron, O., Gal-Yam, A., et al. 2016, ApJ, 818, 3

Kiewe, M., Gal-Yam, A., Arcavi, I., et al. 2012, ApJ, 744, 10

Kobulnicky, H. A., \& Kewley, L. J. 2004, ApJ, 617, 240

Kochanek, C. S. 2009, ApJ, 707, 1578

Kochanek, C. S. 2018, MNRAS, 473, 1633

Kulkarni, S. R. 2013, ATel, 4807, 1

Kuncarayakti, H., Anderson, J. P., Galbany, L., et al. 2018, A\&A, 613, A35

Laher, R. R., Surace, J., Grillmair, C. J., et al. 2014, PASP, 126, 674

Lagattuta, D. J., Mould, J. R., Staveley-Smith, L., et al. 2013, ApJ, 771, 88

Law, N. M., Kulkarni, S. R., Dekany, R. G., et al. 2009, PASP, 121, 1395

Leloudas, G., Hsiao, E. Y., Johansson, J., et al. 2015, A\&A, 574, A61

Leonard, D. C., Filippenko, A. V., Barth, A. J., \& Matheson, T. 2000, ApJ, 536, 239

Leonard, D. C., Sheehan, P., McCarthy, D., et al. 2015, ATel, 7690, 1

Li, W., Filippenko, A. V., Van Dyk, S. D., et al. 2002, PASP, 114, 403

Li, W., Leaman, J., Chornock, R., et al. 2011, MNRAS, 412, 1441

Liddle, A. R. 2004, MNRAS, 351, L49

Liu, Q.-Z., Hu, J.-Y., Hang, H.-R., et al. 2000, A\&AS, 144, 219

Malmquist, K. G. 1922, Meddelanden från Lunds astronomiska observatorium Serie I, 100,

Mauerhan, J. C., Smith, N., Silverman, J. M., et al. 2013a, MNRAS, 431, 2599

Mauerhan, J. C., Smith, N., Filippenko, A. V., et al. 2013b, MNRAS, 430, 1801

Mauerhan, J. C., Van Dyk, S. D., Graham, M. L., et al. 2015, MNRAS, 447, 1922

Margutti, R., Milisavljevic, D., Soderberg, A. M., et al. 2014, ApJ, 780, 21

Martin, J. C., Hambsch, F.-J., Margutti, R., et al. 2015, AJ, 149, 9

van Marle, A. J., Smith, N., Owocki, S. P., \& van Veelen, B. 2010, MNRAS, 407, 2305

Masci, F. J., Papadogiannakis, S., Laher, R. R., et al. 2015, Generating Light Curves from Forced PSF-fit Photometry on PTFIDE Difference-images. Version 2015 August 11

Masci, F. J., Laher, R. R., Rebbapragada, U. D., et al. 2017, PASP, 129, 014002

Mould, J. R., Huchra, J. P., Freedman, W. L., et al. 2000a, ApJ, 529, 786

Mould, J. R., Huchra, J. P., Freedman, W. L., et al. 2000b, ApJ, 545, 547

Moriya, T. J., \& Maeda, K. 2014, ApJ, 790, L16

Moriya, T. J., Maeda, K., Taddia, F., et al. 2014, MNRAS, 439, 2917

Moriya, T. J., Sorokina, E. I., \& Chevalier, R. A. 2018, Space Sci. Rev., 214, 59

Nadyozhin, D. K. 1994, ApJS, 92, 527

Nicholl, M., Walton, N., Blagorodnova, N., et al. 2013, ATel, 4860, 1

Nyholm, A., Sollerman, J., Taddia, F., et al. 2017, A\&A, 605, A6

Ochsenbein, F., Bauer, P., \& Marcout, J. 2000, A\&AS, 143, 23

Ofek, O. 2012, Cent. Bur. Electron. Telegr., 3313,

Ofek, E. O. 2014, Astrophysics Source Code Library [record ascl:1407.005]

Ofek, E. O., Laher, R., Law, N., et al. 2012, PASP, 124, 62

Ofek, E. O., Sullivan, M., Cenko, S. B., et al. 2013, Nature, 494, 65

Ofek, E. O., Sullivan, M., Shaviv, N. J., et al. 2014a, ApJ, 789, 104

Ofek, E. O., Zoglauer, A., Boggs, S. E., et al. 2014b, ApJ, 781, 42

Ofek, E. O., Arcavi, I., Tal, D., et al. 2014c, ApJ, 788, 154

Papadogiannakis, S., Goobar, A., Amanullah, R., et al. 2019, MNRAS, 483, 5045

Patat, F., Taubenberger, S., Benetti, S., Pastorello, A., \& Harutyunyan, A. 2011, A\&A, 527, L6

Pastorello, A., Turatto, M., Benetti, S., et al. 2002, MNRAS, 333, 27

Pastorello, A., Kochanek, C. S., Fraser, M., et al. 2018, MNRAS, 474, 197

Perley, D. A., Quimby, R. M., Yan, L., et al. 2016, ApJ, 830, 13

Petrosian, V. 1976, ApJ, 209, L1

Phillips, M. M. 1993, ApJ, 413, L105

Pskovskii, I. P. 1977, Sov. Astron., 21, 675

Reilly, E., Maund, J. R., Baade, D., et al. 2017, MNRAS, 470, 1491

Richardson, D., Branch, D., Casebeer, D., et al. 2002, AJ, 123, 745 
Richardson, D., Jenkins, R. L., III, Wright, J., \& Maddox, L. 2014, AJ, 147, 118 Rubin, A., Gal-Yam, A., De Cia, A., et al. 2016, ApJ, 820, 33

Sana, H., de Mink, S. E., de Koter, A., et al. 2012, Science, 337, 444

Schlafly, E. F., \& Finkbeiner, D. P. 2011, ApJ, 737, 103

Schlegel, E. M. 1990, MNRAS, 244, 269

Schulze, S., Krühler, T., Leloudas, G., et al. 2018, MNRAS, 473, 1258

Schwarz, D. H., \& Pringle, J. E. 1996, MNRAS, 282, 1018

Silverman, J. M., Nugent, P. E., Gal-Yam, A., et al. 2013, ApJS, 207, 3

Sollerman, J., Cumming, R. J., \& Lundqvist, P. 1998, ApJ, 493, 933

Sollerman, J., Mörtsell, E., Davis, T. M., et al. 2009, ApJ, 703, 1374

Soumagnac, M. T., Ofek, E. O., Gal-yam, A., et al. 2019, ApJ, 872, 141

Smith, N. 2014, ARA\&A, 52, 487

Smith, N. 2017a, Handbook of Supernovae (Springer International Publishing AG), 403

Smith, N. 2017b, Philos. Trans. R. Soc. London Ser. A, 375, 20160268

Smith, N., Li, W., Foley, R. J., et al. 2007, ApJ, 666, 1116

Smith, N., Miller, A., Li, W., et al. 2010, AJ, 139, 1451

Smith, N., Li, W., Silverman, J. M., Ganeshalingam, M., \& Filippenko, A. V. 2011, MNRAS, 415, 773

Smith, N., Mauerhan, J. C., \& Prieto, J. L. 2014, MNRAS, 438, 1191

Smith, N., Andrews, J. E., Mauerhan, J. C., et al. 2016, MNRAS, 455, 3546

Stathakis, R. A., \& Sadler, E. M. 1991, MNRAS, 250, 786

Stoll, R., Prieto, J. L., Stanek, K. Z., \& Pogge, R. W. 2013, ApJ, 773, 12

Stritzinger, M., Taddia, F., Fransson, C., et al. 2012, ApJ, 756, 173

Taubenberger, S., Pastorello, A., Mazzali, P. A., et al. 2006, MNRAS, 371 1459

Taddia, F., Stritzinger, M. D., Sollerman, J., et al. 2013, A\&A, 555, A10

Taddia, F., Sollerman, J., Fremling, C., et al. 2015a, A\&A, 580, A131

Taddia, F., Sollerman, J., Leloudas, G., et al. 2015b, A\&A, 574, A60

Taddia, F., Sollerman, J., Fremling, C., et al. 2019, A\&A, 621, A71

Tartaglia, L., Pastorello, A., Sollerman, J., et al. 2020, A\&A, 635, A39

Tremonti, C. A., Heckman, T. M., Kauffmann, G., et al. 2004, ApJ, 613, 898

Turatto, M., Cappellaro, E., Danziger, I. J., et al. 1993, MNRAS, 262, 128

Tuthill, P. G., Monnier, J. D., Lawrance, N., et al. 2008, ApJ, 675, 698

Valenti, S., Howell, D. A., Stritzinger, M. D., et al. 2016, MNRAS, 459, 3939

Weis, K. 2011, Active OB Stars: Structure, Evolution, Mass Loss, and Critical Limits, 272, 372

Van Dyk, S. D., \& Matheson, T. 2012, Eta Carinae and the Supernova Impostors (Berlin: Springer), 384, 249

Van Dyk, S. D., Peng, C. Y., King, J. Y., et al. 2000, PASP, 112, 1532

Villar, V. A., Berger, E., Metzger, B. D., \& Guillochon, J. 2017, ApJ, 849, 70
Wyrzykowski, L., Udalski, A., \& Kozlowski, S. 2013, ATel, 4859, 1 Yaron, O., \& Gal-Yam, A. 2012, PASP, 124, 668

Young, D. 2016, Transient Name Server Discovery Report, 61

1 Department of Astronomy and the Oskar Klein Centre, Stockholm University, AlbaNova 10691 Stockholm, Sweden

e-mail: anders.nyholm@astro.su.se

2 Division of Physics, Mathematics, and Astronomy, California Institute of Technology, Pasadena, CA 91125, USA

3 Department of Astrophysics/IMAPP, Radboud University, Houtlaan 4, 6525 XZ Nijmegen, The Netherlands

4 Department of Astronomy, University of California, Berkeley, CA 94720-3411, USA

5 Miller Senior Fellow, Miller Institute for Basic Research in Science, University of California, Berkeley, CA 94720, USA

6 Department of Particle Physics and Astrophysics, Weizmann Institute of Science, 234 Herzl St., Rehovot 76100, Israel

7 Las Cumbres Observatory, 6740 Cortona Drive, Suite 102, Goleta, CA 93117-5575, USA

8 Department of Physics, University of California, Santa Barbara, CA 93106-9530, USA

9 IPAC, California Institute of Technology, 1200 E. California Blvd, Pasadena, CA 91125, USA

10 DTU Space, National Space Institute, Technical University of Denmark, Elektrovej 327, 2800 Kgs. Lyngby, Denmark

11 Department of Physics and the Oskar Klein Centre, Stockholm University, AlbaNova 10691, Stockholm, Sweden

12 National Astronomical Observatory of Japan, 2-21-1 Osawa, Mitaka, Tokyo 181-8588, Japan

13 Benoziyo Center for Astrophysics, Weizmann Institute of Science, 76100 Rehovot, Israel

14 Department of Astronomy/Mount Laguna Observatory, San Diego State University, 5500 Campanile Drive, San Diego, CA 92812-1221, USA

15 Kavli Institute for the Physics and Mathematics of the Universe (WPI), The University of Tokyo Institutes for Advanced Study, The University of Tokyo, Kashiwa, Chiba 277-8583, Japan

16 Jet Propulsion Laboratory, California Institute of Technology, Pasadena, CA 91109, USA 
A. Nyholm et al.: Type IIn SN light-curve properties

Appendix A: Classification spectra and light-curves

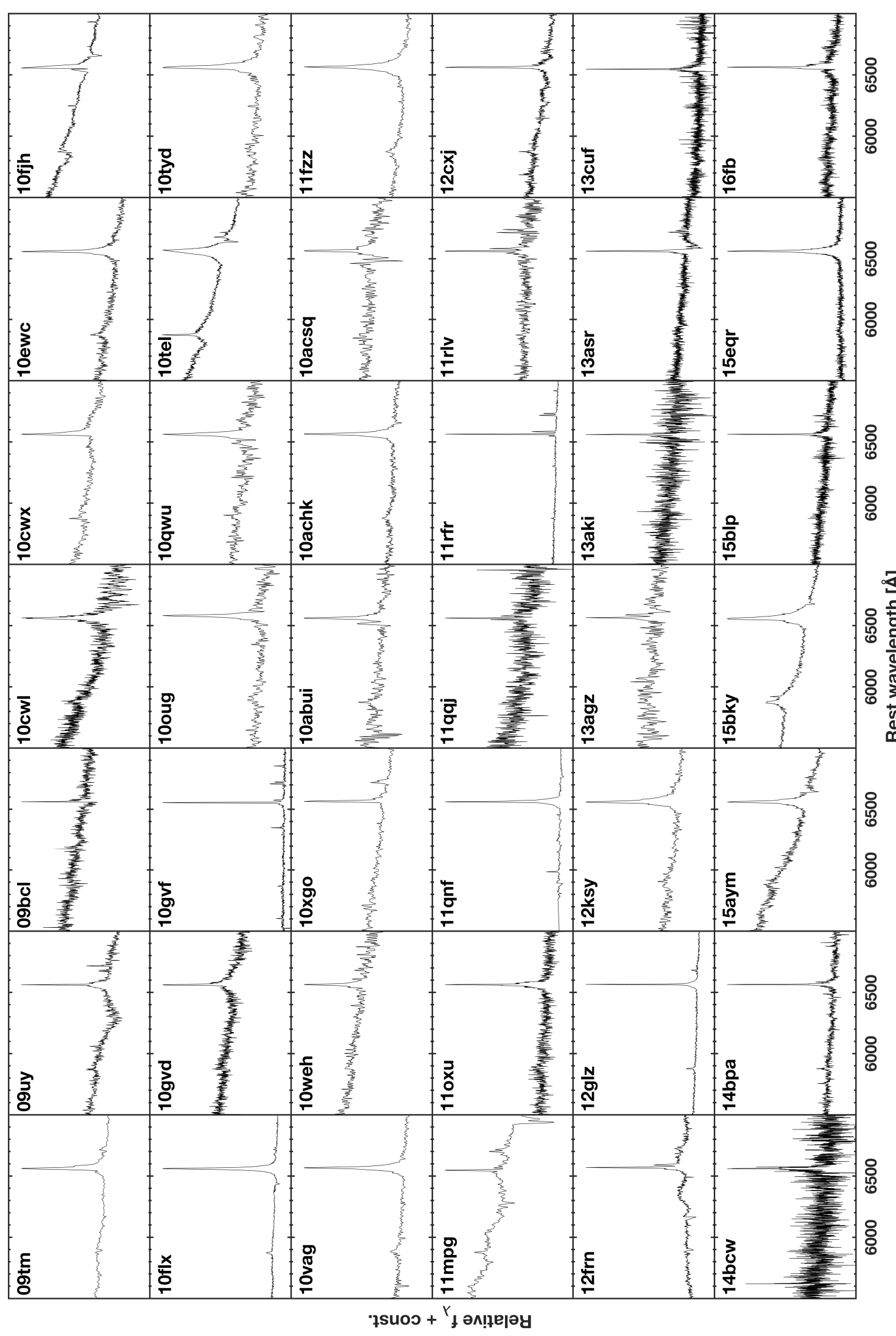

จำ

施要宁

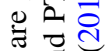

娄

政

s

空政

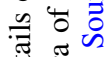

苋

至 की

若

娄离

记

娄

密

承

\&

要兘

है

昰.

制䏝

:

政

辽

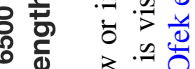

형 3

需 흘

萬 本

$\llbracket \stackrel{0}{\pi}$

bo

言

के

可

空

总.

言 สే

흘

可施

은

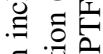

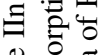

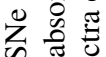

글

政

प응

플

कू ฮ

कै है

क्ष

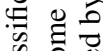

㞼点递

的象

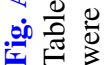




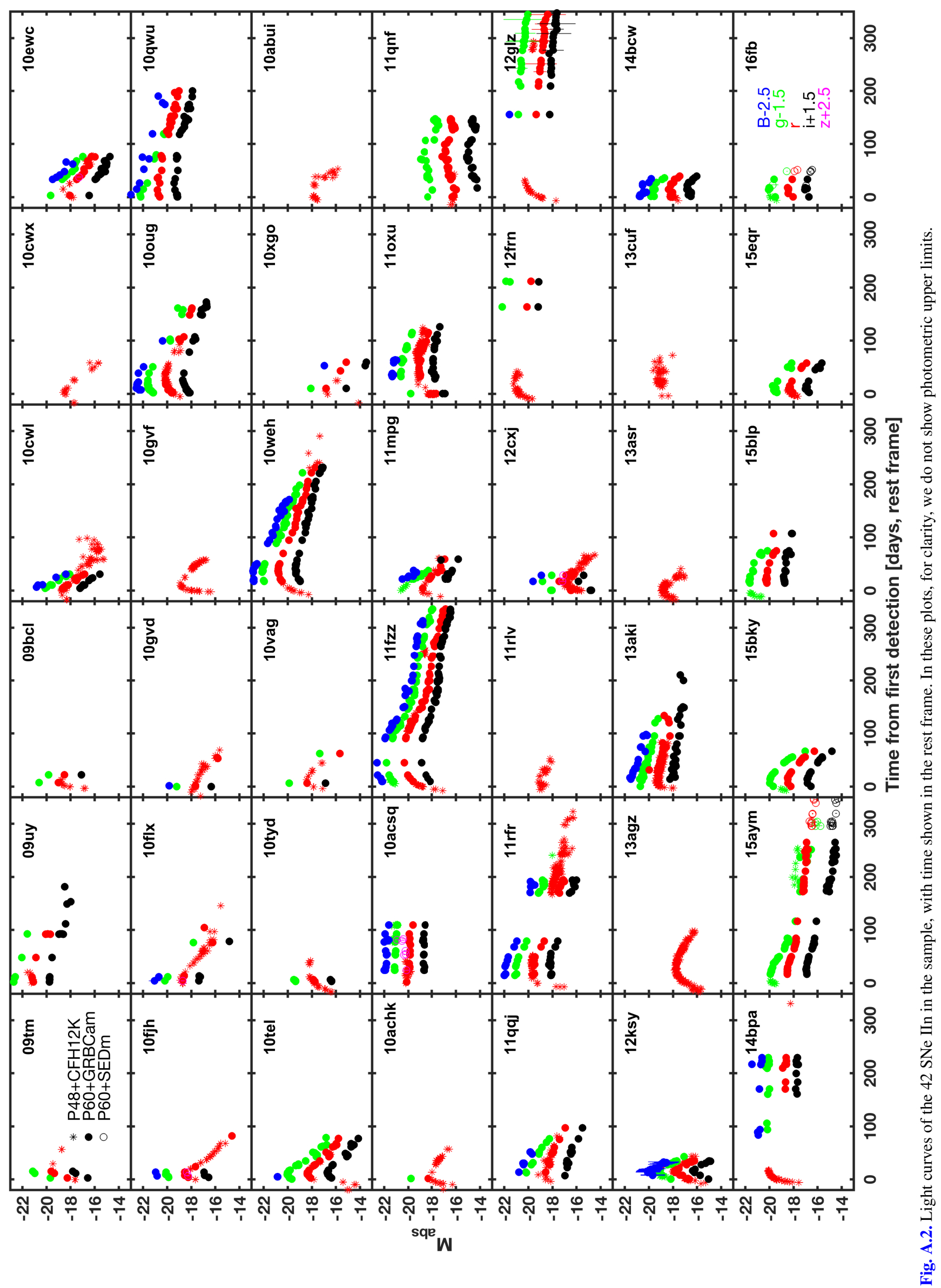



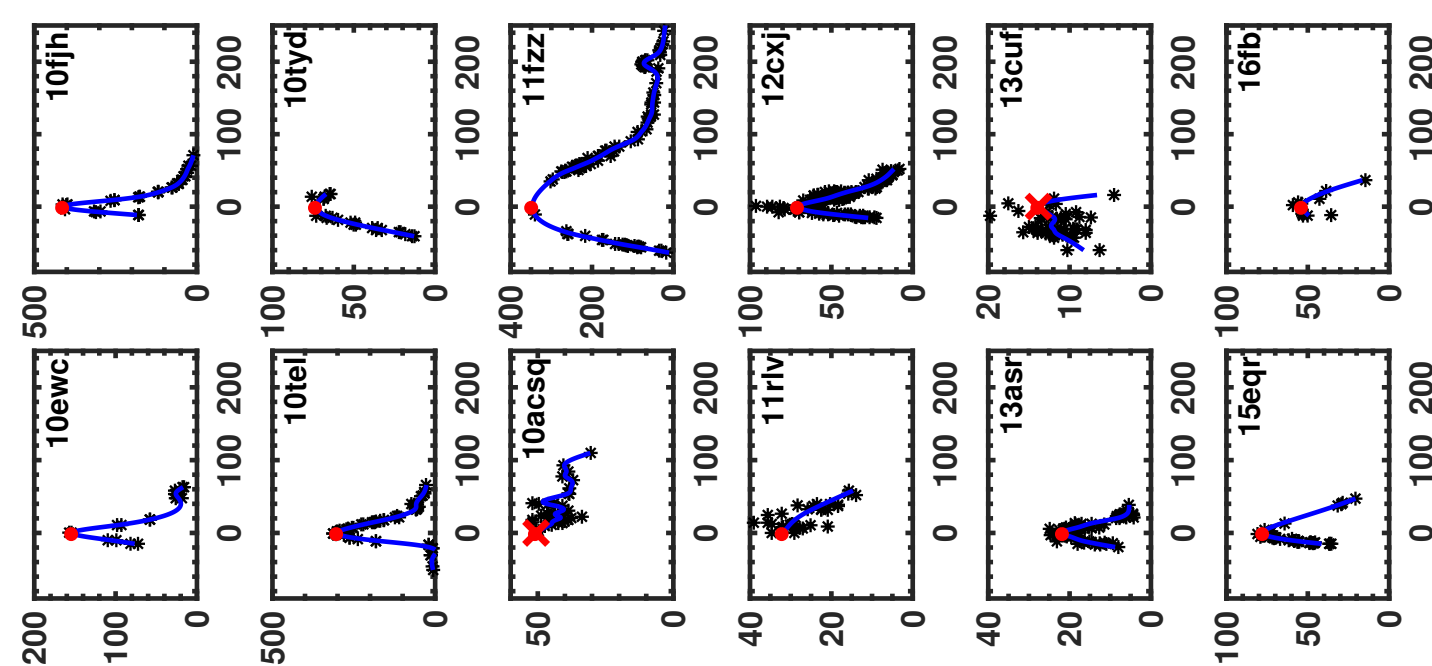

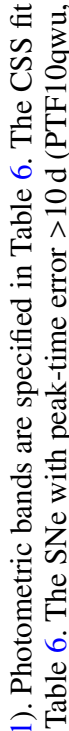
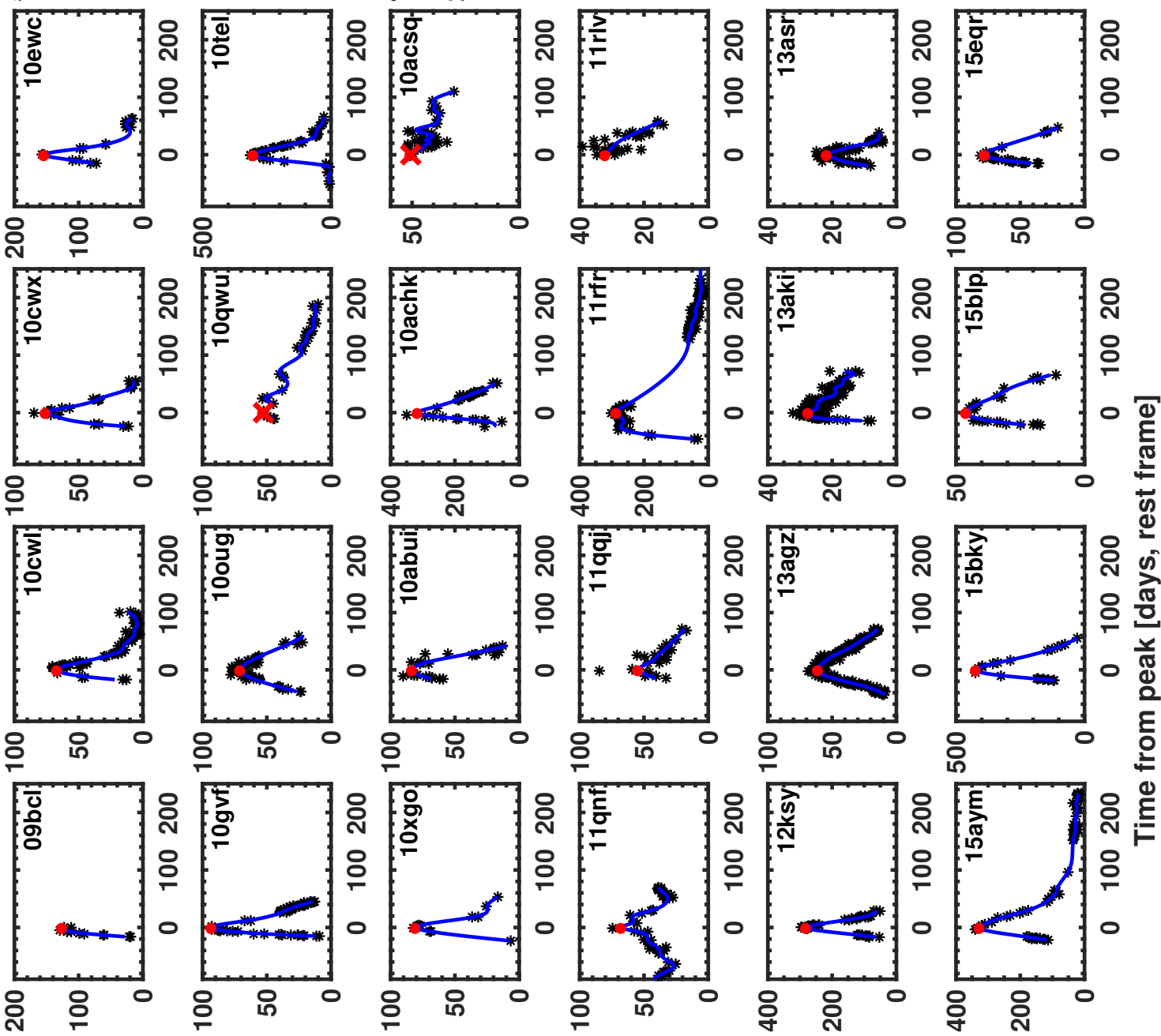

芩

$\stackrel{\mathscr{Q}}{\Xi}$

$\stackrel{0}{\Xi}$

坣

要

원
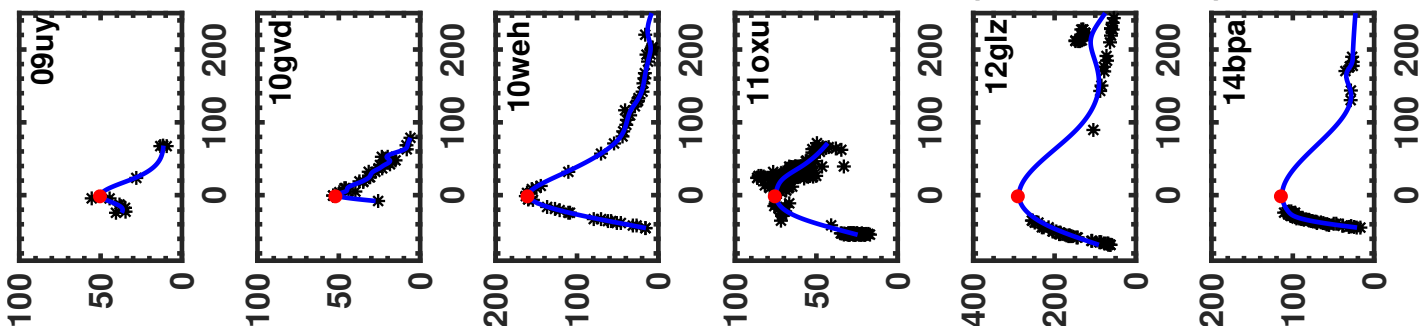

की

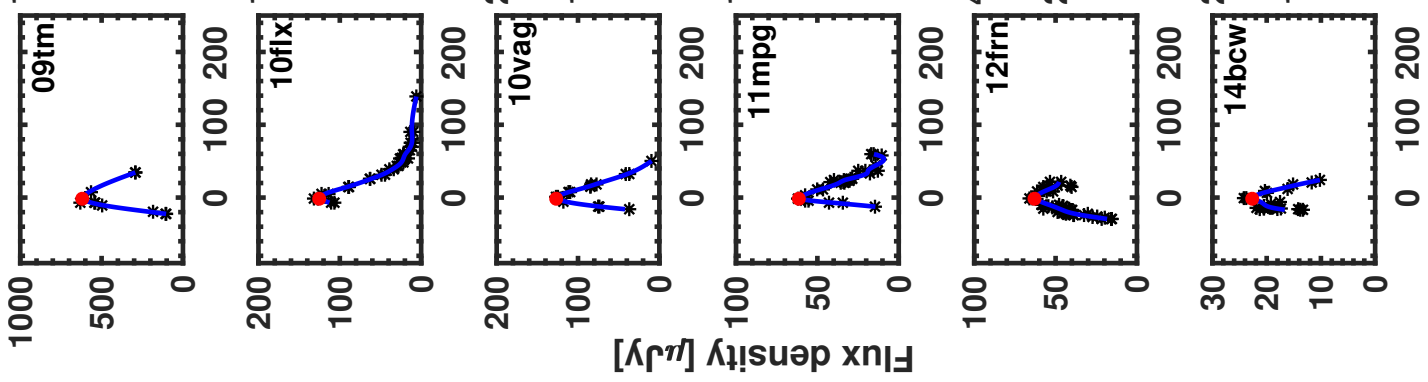



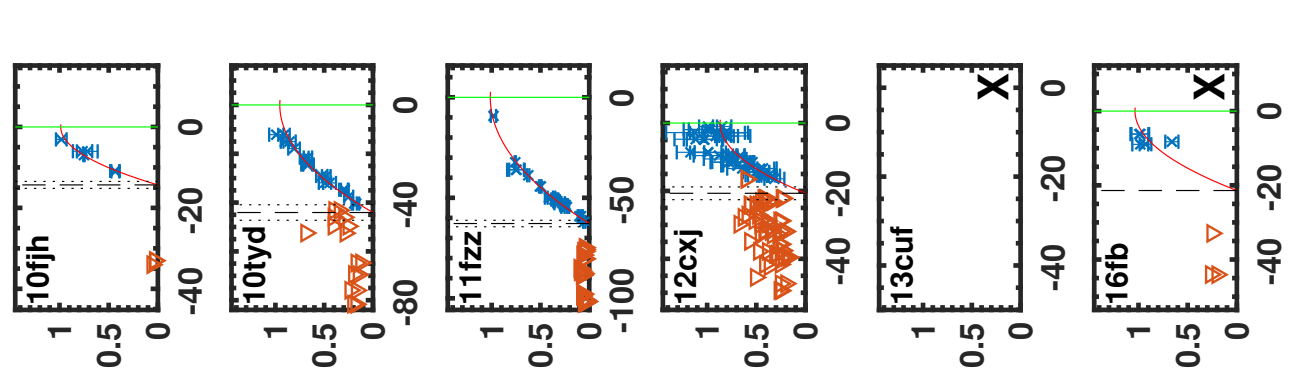

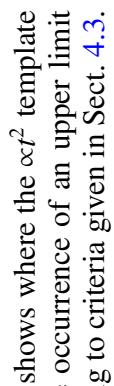
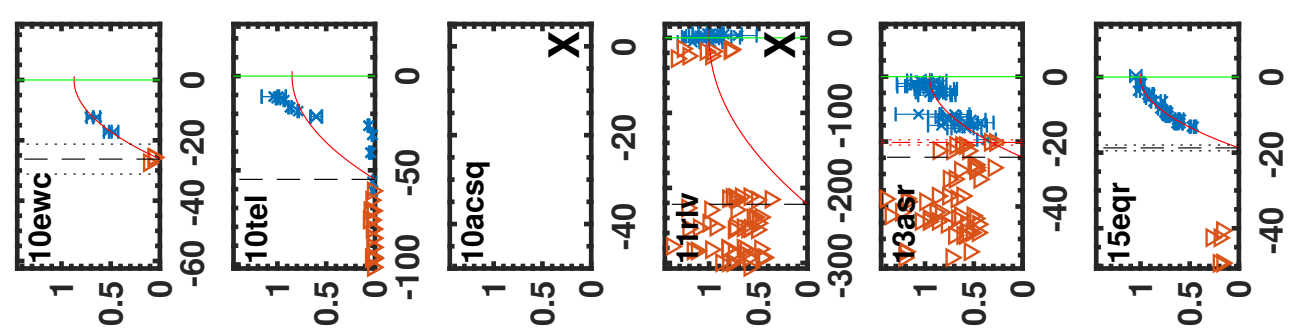

音 量

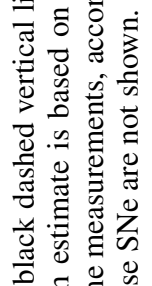
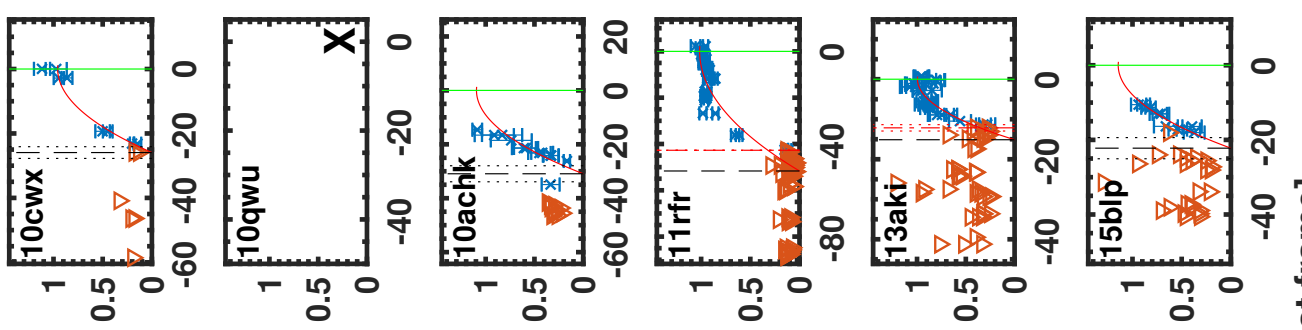

远寻号

$\because$ वे

응

西

"⿹
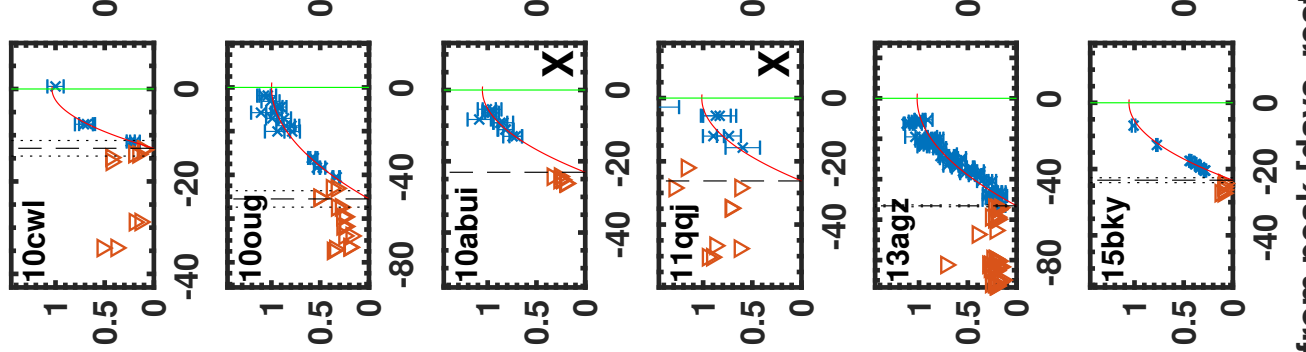

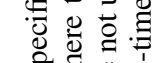
के की

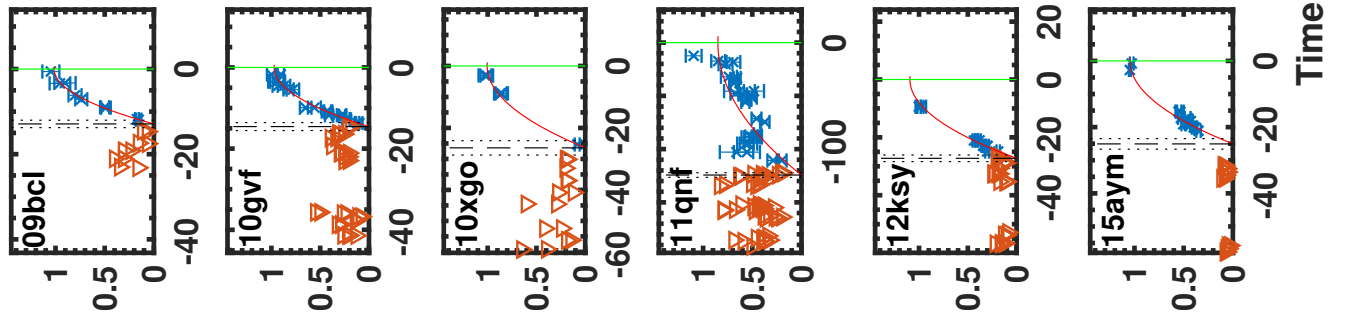

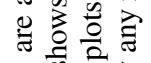

o ${ }^{5}$ :

응

过

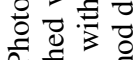

i. 范艺

䁇焉焉

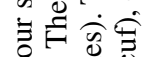

$\Xi \dot{\mathrm{s}} \Xi \mathrm{E}$

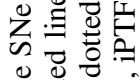

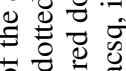

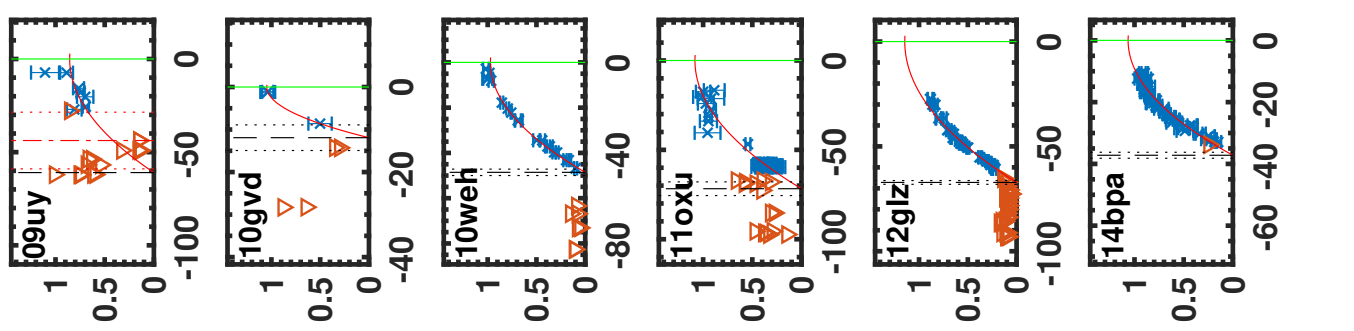

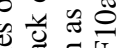

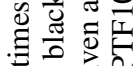

of

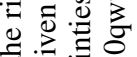

政

跑言吉

员

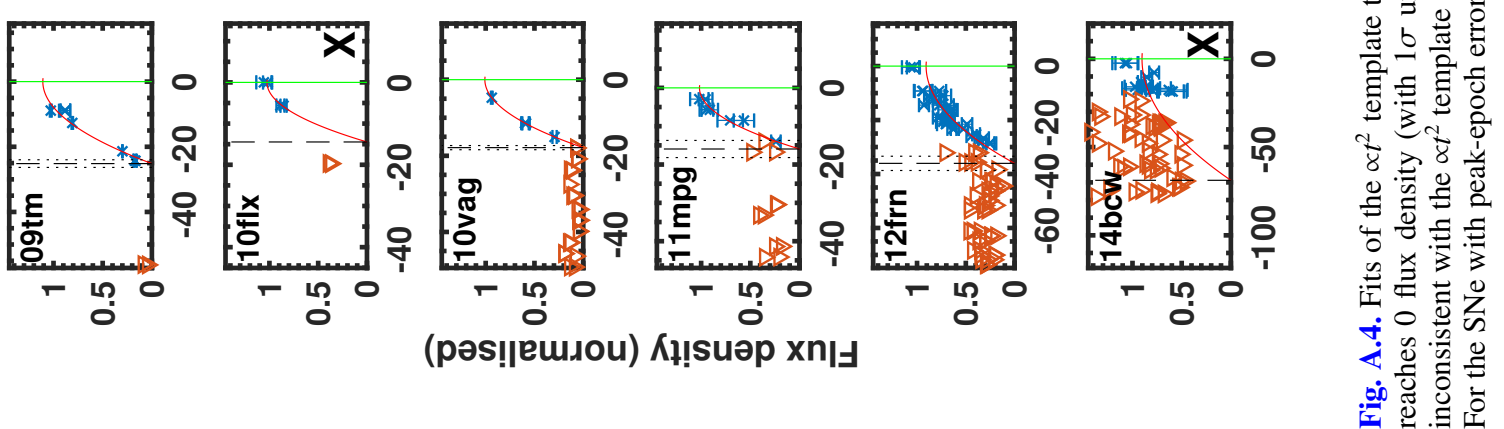




\section{Appendix B: K-corrections for SNe IIn}

$\mathrm{K}$-corrections were determined in the $g, r$, and $i$ bands, while phases refer to the peak of each $\mathrm{SN}^{5}$ (see Sect. 4.1 and Table 6) and are given in the rest frame, hence corrected by a factor $(1+z)$. Owing to the lack of detailed individual spectral sequences, the inferred K-corrections are based on the spectroscopic evolution of the entire sample as a whole, with each spectrum corresponding to a specific phase.

For each event, we take the derived redshift as a reference, shifting all the rest-frame spectra of the sample to that particular $z$ using the IRAF task DOPCOR (called through PYRAF ${ }^{6}$ ). We then compute synthetic magnitudes on the shifted spectra $\left(m_{z, t}\right)$ using the IRAF task CALCPHOT included in the software package Space Telescope Science Data Analysis System (STSDAS) and K-corrections $\left(m_{z, t}-m_{z_{0}, t}\right.$, where $m_{z_{0}, t}$ are the synthetic magnitudes computed on the rest-frame spectra). All spectra were previously corrected for the foreground MW extinction along the line of sight and, for each filter, we did not consider spectra without the sufficient spectral coverage (i.e., not covering the entire filter bandwidth) after applying the specific redshift correction.

In Fig. B.1 we show the derived K-corrections for each redshift, also including those obtained from other SNe IIn with well-sampled spectroscopic evolutions available in the literature (SNe 1988Z, 1998S, 2010jl, and 2015da; Turatto et al. 1993; Fassia et al. 2001; Fransson et al. 2014; Tartaglia et al. 2020). Final values were inferred fitting third-order polynomials to the $\mathrm{K}$-corrections computed at each epoch and $1 \sigma$ errors were assumed as uncertainties. We find corrections of $\lesssim 0.2$ mag at all phases up to $z \approx 0.1$, while at higher redshifts we start to see a significant scatter in the data points.
Interpolating the derived polynomials at peak brightness and $+50 \mathrm{~d}$, we can estimate the effects of the K-corrections on the photometric properties of our sample (see Fig. B.2). A LSQ fourth-order fit to the $r$-band $\mathrm{K}$-correction at light-curve peak gives us Eq. (B.1), and a LSQ first-order fit to the $r$-band Kcorrection errors at light-curve peak gives us Eq. (B.2):

$$
\begin{aligned}
& K_{r}(z)=184.16 z^{4}-86.69 z^{3}+8.96 z^{2}+0.95 z+0.01 \mathrm{mag}, \\
& \text { and } K_{r}^{\operatorname{Err}}(z)=0.89 z-0.01 \mathrm{mag} .
\end{aligned}
$$

Equations (B.1) and (B.2) are valid for $z \leq 0.3135$. The errors used for Eq. (B.2) are the errors of the fits in Fig. B.1. The data used to make the fits for Eqs. (B.1) and (B.2) are given in Table B.1.

At maximum, we see an increase in the K-correction up to $z \approx 0.15$, followed by a flattening around $z \approx 0.2$, while at higher redshifts we see a further increase up to $\sim 0.3 \mathrm{mag}$ (although the derived correction at these redshifts is affected by the large scatter in the data points and the lower number of available spectra, owing to the limited spectral coverage). We find a similar evolution in the K-corrections estimated at $+50 \mathrm{~d}$, although we do not notice the flattening observed at maximum, while we see a steep decline from $\sim 0.18$ to $\sim 0.09$ mag in the $0.15 \lesssim z \lesssim 0.2$ range. In Fig. B.2 (inset) we also show the effect of the derived K-corrections on the slope of light-curves in the first $50 \mathrm{~d}$ after maximum brightness. We note a maximum deviation of $-1.5 \times 10^{-3}$ mag at $z \approx 0.25$ in the derived slope, suggesting a negligible contribution of the K-correction up to $z \approx 0.3$, while it starts to play an important role in determining magnitudes at peak at $z \gtrsim 0.15$.

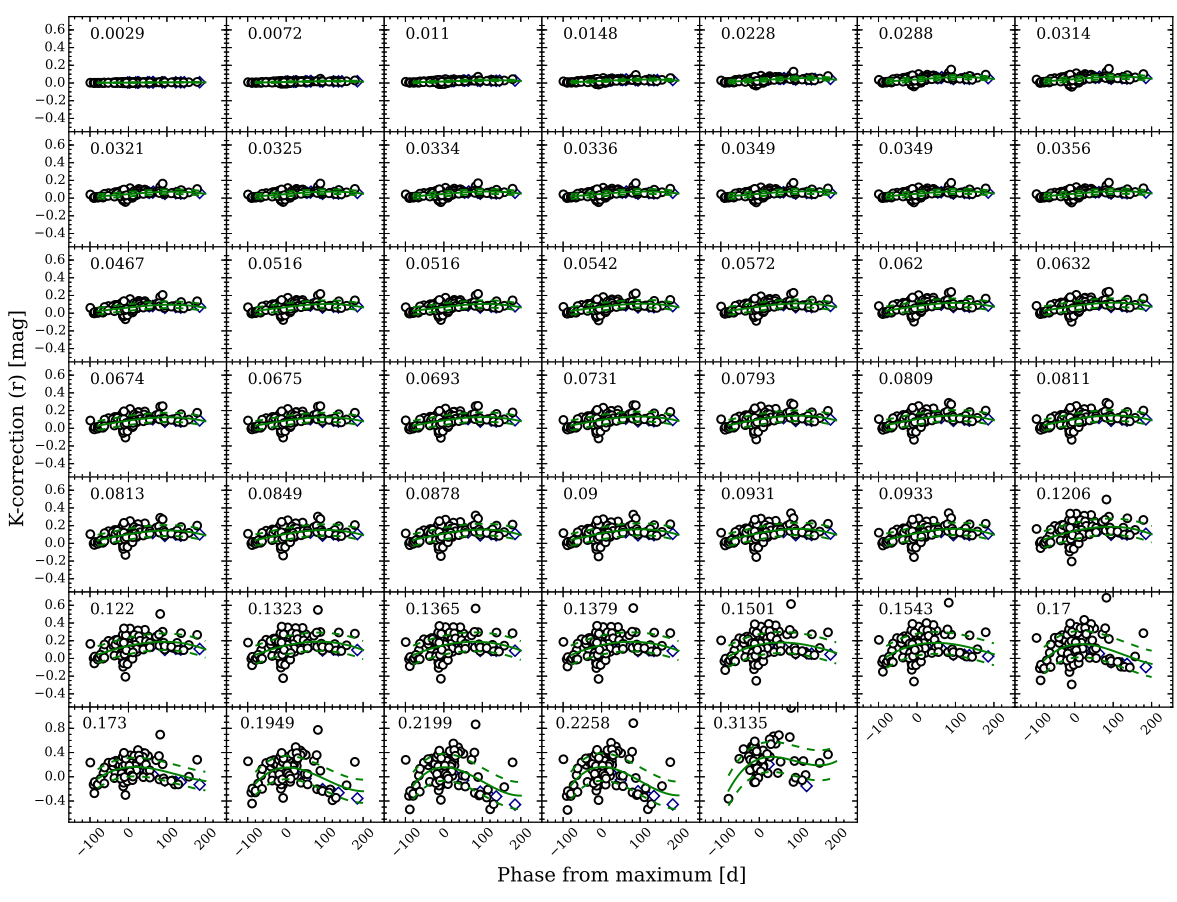

Fig. B.1. K-corrections in the $r$-band for $\mathrm{SNe}$ IIn at different redshifts. Blue points are the corrections computed for well-studied SNe IIn available in the literature (see the text for more details). Green lines are the best-fit models obtained using third-order polynomials, while dashed lines are the $1 \sigma$ uncertainties of each fit. Phases (rest frame) refer to the estimated light-curve peak (Sect. 4.1 and Table 6) of each SN in the sample.

\footnotetext{
To allow better sampling of the K-correction at higher redshifts, the SNe PTF10qwu, PTF10acsq and iPTF13cuf (excluded in the main analanalysis, cf. Sect. 4.1) are included here, with peak epochs approximated as shown by the " $\mathrm{X}$ " symbols in (Fig. A.3).

6 https://github.com/spacetelescope/pyraf
} 
Table B.1. K-corrections ( $r$-band) for SNe IIn.

\begin{tabular}{|c|c|c|c|c|}
\hline Redshift & $\begin{array}{c}K_{0 \mathrm{~d}} \\
(\mathrm{mag})\end{array}$ & $\begin{array}{l}\text { Error }(0 \mathrm{~d}) \\
\quad(\mathrm{mag})\end{array}$ & $\begin{array}{c}K_{50 \mathrm{~d}} \\
(\mathrm{mag})\end{array}$ & $\begin{array}{c}\text { Error }(50 \mathrm{~d}) \\
(\mathrm{mag})\end{array}$ \\
\hline 0.0029 & 0.005 & 0.003 & 0.007 & 0.003 \\
\hline 0.0072 & 0.012 & 0.007 & 0.017 & 0.007 \\
\hline 0.0110 & 0.017 & 0.010 & 0.025 & 0.010 \\
\hline 0.0148 & 0.023 & 0.013 & 0.033 & 0.013 \\
\hline 0.0228 & 0.034 & 0.019 & 0.047 & 0.019 \\
\hline 0.0288 & 0.042 & 0.024 & 0.057 & 0.024 \\
\hline 0.0314 & 0.045 & 0.026 & 0.061 & 0.026 \\
\hline 0.0321 & 0.046 & 0.026 & 0.062 & 0.026 \\
\hline 0.0325 & 0.047 & 0.027 & 0.063 & 0.027 \\
\hline 0.0334 & 0.048 & 0.027 & 0.065 & 0.027 \\
\hline 0.0336 & 0.048 & 0.028 & 0.065 & 0.028 \\
\hline 0.0349 & 0.050 & 0.029 & 0.067 & 0.029 \\
\hline 0.0356 & 0.051 & 0.029 & 0.068 & 0.029 \\
\hline 0.0467 & 0.064 & 0.037 & 0.085 & 0.037 \\
\hline 0.0516 & 0.070 & 0.041 & 0.093 & 0.041 \\
\hline 0.0542 & 0.073 & 0.043 & 0.096 & 0.043 \\
\hline 0.0572 & 0.076 & 0.045 & 0.100 & 0.045 \\
\hline 0.0620 & 0.081 & 0.049 & 0.106 & 0.049 \\
\hline 0.0632 & 0.082 & 0.049 & 0.108 & 0.049 \\
\hline 0.0674 & 0.088 & 0.052 & 0.113 & 0.052 \\
\hline 0.0675 & 0.088 & 0.052 & 0.114 & 0.052 \\
\hline 0.0693 & 0.090 & 0.053 & 0.116 & 0.053 \\
\hline 0.0731 & 0.095 & 0.055 & 0.122 & 0.055 \\
\hline 0.0793 & 0.101 & 0.060 & 0.130 & 0.060 \\
\hline 0.0809 & 0.103 & 0.061 & 0.133 & 0.061 \\
\hline 0.0811 & 0.103 & 0.061 & 0.133 & 0.061 \\
\hline 0.0813 & 0.104 & 0.061 & 0.133 & 0.061 \\
\hline 0.0849 & 0.107 & 0.064 & 0.138 & 0.064 \\
\hline 0.0878 & 0.110 & 0.066 & 0.142 & 0.066 \\
\hline 0.0900 & 0.113 & 0.068 & 0.145 & 0.068 \\
\hline 0.0931 & 0.116 & 0.070 & 0.150 & 0.070 \\
\hline 0.0933 & 0.116 & 0.070 & 0.150 & 0.070 \\
\hline 0.1206 & 0.140 & 0.093 & 0.177 & 0.093 \\
\hline 0.1220 & 0.141 & 0.094 & 0.178 & 0.094 \\
\hline 0.1323 & 0.147 & 0.103 & 0.182 & 0.103 \\
\hline 0.1365 & 0.150 & 0.106 & 0.183 & 0.106 \\
\hline 0.1379 & 0.151 & 0.107 & 0.183 & 0.107 \\
\hline 0.1501 & 0.155 & 0.119 & 0.180 & 0.119 \\
\hline 0.1543 & 0.156 & 0.124 & 0.176 & 0.124 \\
\hline 0.1700 & 0.158 & 0.148 & 0.148 & 0.148 \\
\hline 0.1730 & 0.156 & 0.154 & 0.146 & 0.154 \\
\hline 0.1949 & 0.152 & 0.192 & 0.098 & 0.192 \\
\hline 0.2199 & 0.157 & 0.223 & 0.086 & 0.223 \\
\hline 0.2258 & 0.161 & 0.227 & 0.092 & 0.227 \\
\hline 0.3135 & 0.295 & 0.248 & 0.321 & 0.248 \\
\hline
\end{tabular}

Notes. The K-correction at light-curve peak is called $K_{0 \mathrm{~d}}$, at $50 \mathrm{~d}$ (rest frame) after peak it is called $K_{50 \mathrm{~d}}$. The data in this table are plotted in Fig. B.2.

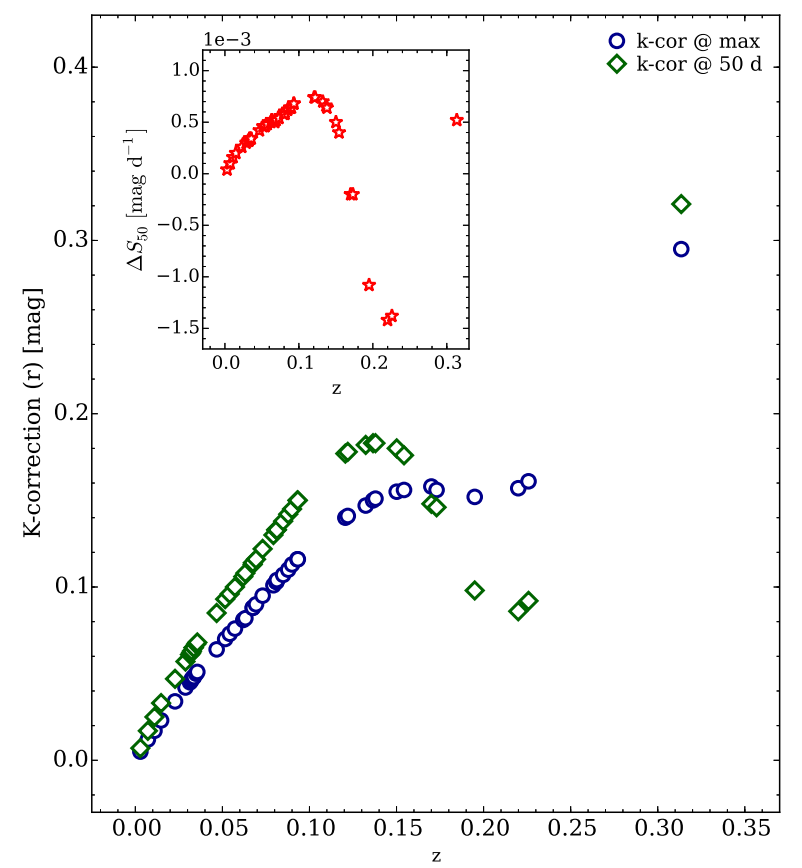

Fig. B.2. Effect of the K-correction on photometric properties of our SN IIn sample. The required correction is smaller than $\sim 0.2$ mag in both peak magnitudes and $\operatorname{mag}_{50 \mathrm{~d}}$ at redshifts $\lesssim 0.15$, although it can be as high as $0.3 \mathrm{mag}$ at $z \gtrsim 0.3$. The inset shows the effects of the $\mathrm{K}$-correction on the light-curve slopes measured from peak to $+50 \mathrm{~d}$, which is smaller than $1.5 \times 10^{-3} \mathrm{mag}$ for all the redshifts of our sample. 